\title{
Attentional bias and anxiety: conceptual issues and empirical data
}

Citation for published version (APA):

Lavy, E. H. (1993). Attentional bias and anxiety: conceptual issues and empirical data. [Doctoral Thesis, Maastricht University]. Rijksuniversiteit Limburg. https://doi.org/10.26481/dis.19931223el

Document status and date:

Published: 01/01/1993

DOI:

$10.26481 /$ dis. $19931223 \mathrm{el}$

Document Version:

Publisher's PDF, also known as Version of record

\section{Please check the document version of this publication:}

- A submitted manuscript is the version of the article upon submission and before peer-review. There can be important differences between the submitted version and the official published version of record.

People interested in the research are advised to contact the author for the final version of the publication, or visit the DOI to the publisher's website.

- The final author version and the galley proof are versions of the publication after peer review.

- The final published version features the final layout of the paper including the volume, issue and page numbers.

Link to publication

\footnotetext{
General rights rights.

- You may freely distribute the URL identifying the publication in the public portal. please follow below link for the End User Agreement:

www.umlib.nl/taverne-license

Take down policy

If you believe that this document breaches copyright please contact us at:

repository@maastrichtuniversity.nl

providing details and we will investigate your claim.
}

Copyright and moral rights for the publications made accessible in the public portal are retained by the authors and/or other copyright owners and it is a condition of accessing publications that users recognise and abide by the legal requirements associated with these

- Users may download and print one copy of any publication from the public portal for the purpose of private study or research.

- You may not further distribute the material or use it for any profit-making activity or commercial gain

If the publication is distributed under the terms of Article $25 \mathrm{fa}$ of the Dutch Copyright Act, indicated by the "Taverne" license above, 
Attentional bias and anxiety:

Conceptual issues and empirical data 
+ $\quad:$ 


\section{Attentional bias and anxiety:}

\section{Conceptual issues and empirical data}

\section{PROEFSCHRIFT}

ter verkrijging van de graad van doctor aan de Rijksuniversiteit Limburg te Maastricht, op gezag van de Rector Magnificus, Prof. Dr. H. Philipsen, volgens het besluit van het College van Dekanen, in het openbaar te verdedigen op donderdag, 23 december 1993 om 14.00 uur

$$
\text { door }
$$

Edith Hanna Lavy 


\title{
Promotor
}

Prof. Dr. M.A. van den Hout

\author{
Beoordelingscommissie \\ Prof. Dr. J.J.C.B. Bremer (voorzitter) \\ Prof. Dr. P.M.G. Emmelkamp (Rijksuniversiteit Groningen) \\ Prof. Dr. N.H. Frijda (Universiteit van Amsterdam) \\ Prof. Dr. J. Jolles \\ Prof. Dr. H.G. Schmidt
}

Ontwerp en realisatie omslag

InduComm, IJmuiden, the Netherlands

\section{Druk}

Datawyse Maastricht / Krips Repro Meppel

CIP-DATA KONINKLIJKE BIBLIOTHEEK, DEN HAAG

Lavy, Edith

Attentional bias and anxiety: conceptual issues and empirical data / Edith Lavy. - [S.I. : s.n.]

Thesis Maastricht, - With ref. - With a summary in Dutch.

ISBN 90-9006597-0

Subject headings: attentional bias and anxiety. 
Voor René 


\section{Contents}

Chapter 1 Conditioning and behaviour therapy:

A bird-eye's view of their evolution

1.1 Classical conditioning and the two factor 10 theory of anxiety

1.2 Clinical applications of conditioning theory 10

1.3 Criticisms 12

1.4 Opposing classical conditioning: Pragmatism or 13 acceptance of alternative theories

1.5 Adaptation of the classical conditioning theory 15

1.6 Concluding remarks 16

Chapter 2 Cognitive theories and empirical data: Self-reports and information processing experiments

2.1 Cognitive theories 18

2.2 Self-report data 20

2.3 Experimental data 21

2.4 Selective processing in anxiety states: 22

Attentional processes

2.5 Selective processing in anxiety states: 23

Identification and interpretation

2.6 Selective processing in anxiety states: 26

Retrieval processes

2.7 Concluding remarks 32

$\begin{array}{lll}\text { Chapter } & 3 & \text { Attentional bias: Topics of interest }\end{array}$

3.1 Attention, anxiety and performance: 36

Introductory considerations
Measurement of selective attention: Paradigms

$\begin{array}{lll}3.2 & \text { Measurement of selective attention: Paradigms } & 37 \\ 3.3 & \text { Specificity of the Stroop effect: Populations } & 39\end{array}$

3.4 The state-trait problem: Are current mood or 47 enduring factors causal to attentional bias?
The familiarity problem

$\begin{array}{lll}\text { 3.5 The familiarity problem } & 48 \\ 3.6 & \text { Specificity of the Stroop effect: Stimuli } & 49\end{array}$

3.7 Memory bias 50

3.8 The significance of attentional bias 51

3.9 Ecological validity of attentional tests 52

3.10 Summary $\quad 54$ 
Chapter 5 Experimental data

5.1 Attentional bias and spider phobia: Conceptual and clinical issues

5.2 Selective processing of emotional information in obsessive compulsive disorder

5.3 Attentional bias for appetitive cues: Effects of fasting in normal subjects

5.4 Thought suppression induces intrusions

5.5 Cognitive avoidance and attentional bias: Causal relationships

5.6 Attentional bias and facilitated escape:

A pictorial test

5.7 Selective attention evidenced by pictorial and linguistic Stroop tasks

Chapter 6 Recapitulation and general discussion

6.1 Replication of earlier findings

6.2 The reactivity of attentional bias: Transient or enduring phenomena

6.3 Stimulus familiarity

6.4 Stimulus specificity

6.5 Memory bias

6.6 Ecological validity of attentional bias tests

6.7 Adaptiveness of attentional bias: Activation. attention and behaviour

6.8 Attentional bias and cognitive theories

6.9 A speculative integration of findings

6.10 Criltical remarks about the model:

Theoretical considerations

6.11 Critical remarks about the modell:

Clinical implications

6.12 Concluding remarks: Recommendations

for future research

Samenvatting

Summary

References

Dankwoord 



\section{Chapter 1}

\section{Conditioning theory and behaviour therapy: A bird-eye's view of their evolution}

This introductory chapter presents a brief survey of the origins and development af conditioning theory and its clinical applications. The first section deals with classical and operant conditioning and Mowrer's two-factor theory of anxiety. In the subsequent sections it is argued that, although the benefits to be derived from behavioural treatment procedures based on classical conditioning theory have been generally recognized, the theory itself appears to suffer from several anomalies. These anomalies are resolved by (1) a nontheoretical application of behavioural techniques, (2) adoption of alternative theories, or (3) accommodation of the traditional classical conditioning theory to modern insights. Several alternative and neoclassical conditioning theories are reviewed and their contribution to the resolution of these anomalies is briefly discussed. The chapter concludes by stating that, whatever position was adopted to the anomalies in classical conditioning theory, cognitive approaches became more accepted in behaviour theory and therapy. 


\subsection{Classical conditioning and the two-factor theory of anxiety}

An introduction to the well-known principles of conditioning would seem a prerequisite of a survey of conditioning theory and behaviour therapy. Since these can be found in any introductory book on learning theories and behaviour therapy le.g. Orlemans, 1985), however, only brief mention will be made of them here.

The study of classical conditioning began with Pavlov's famous experiments, in which he investigated the salivation reflex in dogs. Pavlov paired a neutral stimulus (a particular noise) with a stimulus that naturally led to a salivation reflex (meat powder). After repeated paired presentations of noise and meatpowder, the dogs started to salivate as soon as they heard that particular noise. According to Pavlov, the new salivation reflex was the result of a new learned association between noise and salivation. The meatpowder, naturally associated with the salivation response, was called the 'unconditioned" stimulus (US), the previous neutral noise the 'conditioned" stimulus (CS). Likewise, the salivation reflexes were referred to as 'unconditioned (UR) and 'conditioned' (CR) responses. Although the first study of classical conditioning was carried out with appetitive unconditioned stimuli, the stress of classical conditioning experiments has been on aversive, threatening unconditioned stimuli and their associated responses. Watson and Rayner's (1920) story about the conditioning of a boy, 'Little Albert', is a famous example of how anxiety responses can be learned. From this and more elaborate experiments it has been concluded that the acquisition of neurotic fear can be the result of classical conditioning experiences.

Mowrer (1960) explained the acquisition and maintenance of anxiety by a twofactor theory, in which classical conditioning is combined with operant conditioning. As is known, the essence of operant conditioning is that the frequency of behaviour will increase if the consequences are rewarding, and decrease if the consequences are punishing. According to the two-factor theory, a traumatic event or a range of sub-traumatic events (US) ellicit an anxiety response (UR) in a subject. As a result of classical conditioning, formerly neutral stimuli (CSs) may become associated with the anxiety response, i.e. as a result of classical conditioning the subject will respond to these stimuli with anxiety $(C R)$. Usually, this conditioned anxiety will extinguish when the presentation of a CS is no longer paired with the US. But if the subject avoid confrontation with the CS, the contiguity between CS and US is not dissolved and the subject keeps responding with anxiety to the conditioned stimuli. Although, in the long term, the consequences of avoidance behaviour are definitely counterproductive, in the short term avoidance behaviour serves to reinforce because it immediately reduces anxiety. In brief, according to Mowrer's twa-factor theory, fear acquisition may be due to cllassical conditioning, and fear maintenance to operant conditioning.

\subsection{Clinical applications of conditioning theory}

The clinical implications of experiments into conditioning were recognized almost immediately. As early as Watson and Rayner's (1920) paper on Little Albert, suggestions were being put forward as how anxiety responses might be treated using reconditioning techniques, imitation and habituation. Applying these recommenda- 
tions in the treatment of anxiety in children, Jones (1924) found two treatment methods to be effective in reducing children's fears: associating the feared stimulus with an antagonistic pleasant response and exposing the child to the feared stimulus in the presence of other children who were not afraid. These new insights did not immediately cause a revolution in the treatment of anxiety disorders; in fact several decades would elapse before Jones' findings were reformulated and consolidated into such now well-known treatment procedures as systematic desensitization (Wolpe, 1958) and participant modelling (Bandura, 1977).

The first widely accepted behavioural procedure was Wolpe's systematic desensitization. Based on his research into the experimental induction and elimination of fears in cats, he postulated that fear reduction could be accomplished by the simulia taneous presentation of a threatening stimulus and a "reciprocal inhibitor": a stimu" lus evoking a response antagonistic to anxiety. The reciprocal inhibitor being the stronger of the two stimuli, anxiety would be inhibited and, after repeated presentation, the threatening stimulus would no longer be associated with the anxiety response, but with the antagonistic response. In order to ensure that the inhibitor was indeed stronger than the threatening stimulus, the anxiety-provoking stimuli were presented in a hierarchical way. Wolpe identified several reciprocal inhibitors like food, sexual responses and relaxation responses. For the systematic desensitization procedure Jacobson's (1938) progressive relaxation was used. Wolpe was the first to describe systematically and extensively a behaviour modification procedure that included a sound theoretical basis and, although in later years the theoreticall foundation of systematic desensitization (Emmelkamp, 1975) and the need for relaxation (Marks, 1975) were disputed, his work formed the foundation of modern behavioural treatment programmes.

It was realized gradually that the essential effective component of systematic desensitization was the confrontation with the feared stimulus (Davison, 1968). This appreciation led to major changes in behavioural techniques. Dispensable components such as relaxation were largely dropped, and there was a move away from imaginary techniques to exposure in vivo procedures (Marks, 1987). The rationale for exposure in vivo could be supported by Mowrer"s two-factor theory (Mowrer, $1960)$. According to this theory avoidance and escape behaviours are developed in order to forestall, or at least minimize, confrontation with threatening stimuli. In the short term, these avoidance responses serve to reinforce because anxiety is subsequently reduced, but in the longer term they prevent the conditioned fear from being extinguished. In an exposure in vivo therapy both the classical conditioned association between feared stimuli and anxiety responses and the operant conditioned avoidance behaviour, are treated. Anxious subjects are confronted with the feared stimuli whilst being prevented from carrying out their usual avoidance responses. This exposure will result in an extinction of anxiety responses, and consequently lead to an interruption of the association between conditioned stimuli and anxiety responses.

Exposure in vivo therapy became very popular in the treatment of phoblas. In the sixties and seventies various exposure in vivo programmes for phobias were devel oped and refined, such as gradual exposure in vivo, exposing the subject gradually to a hierarchy of anxiety-provoking stimuli, and massive exposure in vivo or flooding, immediate and prolonged exposure in vivo to the most threatening stimuli (for extensive overviews of the development of behavioural techniques see Emmelkamp (1982) and Marks (1987). 
The effectiveness of behavioural techniques was systematically investigated in controlled therapy effect studies. Exposure in vivo was compared with systematic desensitization and exposure to imaginally presented stimuli (reviewed by Marks, 1987), and proved consistently to be superior to both imaginary techniques. No differences in final improvement were found between gradual exposure in vivo and flooding (Marks, 1987: Yuksel, Marks, Ramm, \& Gosh, 1984), suggesting that it does not matter how the exposure in wivo therapy is given, as long as the subject is exposed to the threatening stimuli and is prevented from escape and avoidance behaviours. In line with these results, standardized exposiure treatments for agoraphobia, simple phobias, social phobia and obsessive compulsive disorder were developed and proved to be as effective (Emmelkamp, 1991) or even more effective (Schulte, Künzel, Pepping, \& Schulte-Bahrenberg, 1992) than tailor-made behaviour therapy. In follow-up studies success rates varied from about $50 \%$ in obsessive compulsive patients and $70-75 \%$ in agoraphobic patients (reviewed by Marks; 1987 ) to about $90 \%$ in animal phobics (Arntz \& Lavy, 1993a; Öst, 1989); exposure in vivo was thus shown to be a very effective method of treating phobias and other anxiety disorders. Because of its effectiveness exposure in wivo, along with behaviour therapy in general, became a widely accepted treatment method for anxiety disorders.

\subsection{Criticisms}

When behaviour therapists had gained the acceptance of their treatment methods, criticism arose from the ranks of the behaviourists themselves. With the increasing application of behavioural techniques, the limits of behaviour therapy became more apparent. At first little was reported about those patients who showed no improvement from behaviour therapy, but gradually behaviour therapists dared to publish their failures (e.g. Foa \& Emmelkamp, 1983). Questions arose: what should be done with patients who refuse to risk any confrontation with the feared stimuli? Or with patients who drop out of therapy because the exposure in vivo sessions do not result in the extinction of anxiety? If conditioning and extinction relate to deterministic laws of learning, why is it that exposure in vivo does not always lead to extinction of anxiety? In trying to find an answer to these and related questions, some behaviour therapists queried the mechanistic appraach that considered an organism as undergoing conditioning experiences rather passively, without taking into account motivational drives, problem-solving capacities and other intrapsychic processes. Therapy based on behaviourism had been an alternative to traditional schools of psychotherapy, but gradually the dissatisfaction with the behaviouristic view of the organism as a "black box" increased.

Rachman extended and formalized this dissatisfaction in a series of critical papers (Rachman, 1976, 1977, 1991). He emphasized that there was little debate about the merits of behaviour therapy, but put forward arguments against acceptance of the conditioning theory of fear. The experience of a frightening event, either in natural circumstances or in a laboratory, does not always prompt the development of an anxiety disorder. As a matter of fact, it usually does not. Even after extremely frightening experiences, such as air raids, only a small number of people develop an anxiety disorder. It also appears to be difficult to create an artificial phobia in the laboratory. Rachman (1977, 1991) mentioned several studies investi- 
gating the effects of aversion therapy and, in contrast to what the conditioning theory suggests, most subjects in these studies did not respond with anxiety to the conditioned stimuli. In short, many failures to condition fear are reported.

The next point at issue is that; according to the conditioning theory, any stimulus has an equal chance of becoming a fear signal. This assumption is in striking contrast to the reality of clinical practice, in which pyjama phobias, grass phobias and electric-outlet phobias (examples from Seligman, 1971 ) are rarely, if ever, seen. Also in epidemiological studies (e.g. Agras, Sylvester, \& Oliveau, 1969) it is demonstrated that in a normal population fear of snakes is twice as common as fear of dentists, although almost certainly contact with the dentist is far more frequent and more likely to be assocliated with aversive experiences. It appears that some stimuli are more likely to be transformed into fear signals than others.

A last issue raised by Rachman was why it is often so difficult to determine the traumatic origin of an anxiety disorder. In many cases, the initiating conditioning event is not excessively traumatic but rather mild, or even completely absent. This does not tally with the theory, since in the traditional conditioning theory, a traumatic experience has to precede the anxiety disorder, resulting in a $\mathrm{CA}$ which can never be stronger than the UCR.

Taking all the arguments together, Rachman, referring to work by Rescorla and Wagner (1972) and by Kamin (1969), pointed out that temporal contiguity between $\mathrm{CS}$ and US is neither sufficient nor necessary to ensure conditioning. Since this contiguity is one of the major principles of the original classical conditioning theory, the weaknesses of this theory were obvious.

\subsection{Opposing classical conditioning: Pragmatism or acceptance of alterna- tive theories}

It was to be expected that behaviourists would react to these anomalies and, in fact, three reaction patterms emerged: nontheoretical pragmatism, acceptance of alternative theories and adaptation of the classical conditioning theory. Thus, the commotion surrounding the anomalies in classical conditioning theory resulted in an effusion of new or renewed theories, a selection of which will be discussed in this section and the next. Tablle 1.1 is a schematical representation of which anomalies were resolved by the various theories reviewed in sections 1.4 and 1.5 .

A group of clinically oriented behaviourists turned their backs on theoretical discussions and responded pragmatically to the discongruence between theory and practice (e.g. Lazarus, 1971). They argued that, since there was empirical evidence for the efficacy of behavioural techniques, these techniques could be emploved regardless of the theoretical basis. This attitude was (rather easily) adopted by clinicians, resulting in an upsurge of 'broad spectrum' treatment methods, not based on conditioning theory, such as coping skills training (Goldfried, 1971; Goldfried \& Trier, 1974), anxiety management training (Suinn, 1976; Suinn \& Richardson, 1971 ) and stress inoculation training (Meichenbaum, 1975, 1977). Cognitive techniques were typically part of these "broad spectrum' approaches, but, like the behavioural techniques, they were used without reference to a theoretical foundation. By its very nature, the pragmatic view did not resolve any of the anomalles. 


\begin{tabular}{|c|c|c|c|}
\hline \multirow[b]{2}{*}{ Theories } & \multicolumn{3}{|c|}{ Anomalies } \\
\hline & $\begin{array}{l}\text { US-CS } \\
\text { parings } \\
\text { without } \\
\text { conditioned fear }\end{array}$ & $\begin{array}{l}\text { CS with conditioned } \\
\text { fear, but without } \\
\text { detectable US }\end{array}$ & $\begin{array}{l}\text { Equipotentiality } \\
\text { and fear } \\
\text { distribution }\end{array}$ \\
\hline No theory/pragrnatism & - & - & - \\
\hline Vicarious learning & + & + & - \\
\hline Social transmission & - & + & - \\
\hline Language conditioning & - & + & + \\
\hline Preparedness hypothesis & $\ldots$ & - & + \\
\hline Incubation theory & - & + & - \\
\hline $\begin{array}{l}\text { Selective conditioning incl. } \\
\text { US revaluation }\end{array}$ & + & + & - \\
\hline
\end{tabular}

Tabje 1.1: Three main anomalies in classical conditioning theory and the contribution of new and reformed theories to the resolution of these anomalies

A second group behaviourists (e.g. Bandura, 1969; Rachman, 1977) embraced alternative theories, in addition to or instead of the classical conditioning theory. Bandura (1969, 1977) emphasized the role of vicarious learning in the acquisition of fears. As early as World War II, Lewis (1942) had observed that children who had been subjected to air-raids were more likely to develop anxiety symptoms if their mothers were frightened. Mineka and her colleagues (Cook, Mineka, Wolkenstein, \& Laitsch, 1985; Mineka, Davidson, Cook, \& Keir, 1984) also showed, in well controlled animal studies, that strong and persistent fear of snakes in rhesus monkeys can be vicariously acquired. Vicarious learning could explain both the non acquisition of fear and the acquisition of fear without the direct experience of a traumatic event. Bandura (1977) further stated that behavioural change would only be realized, when the subject both estimated that the new behaviour would lead to preferred outcomes ('outcome expectations') and expected to be able to carry out that new behaviour' successfully ('efficacy expectations'). According to Bandura, the therapeutic effects of behavioural techniques, like systematic desensitization and exposure in vivo, had to be attributed to the achieved change in efficacy expectations. Rachman (1977) did not reject the conditioning theory, but suggested some additions. He argued that there are at least three pathways to the acquisition of fear: conditioning, vicarious learning and fear acquisition by social transmission, the transference of verbal information and instructions. Whereas the conditioning theory might account for the vicarious acquisition of fear, it is difficult to see how it can explain the social transmission of fears of stimuli that subjects had never encountered. Rachman also accepted Seligman's preparedness hypothesis (1971) as explanation for the non-random distribution of fears. Essence of this hypothesis is that stimuli of evolutionary significance are more likely than other stimuli to become conditioned stimuli for fear. With the expansion of the conditioning theory to include several other theories that had been validated in their own right, several empirical 
findings that had previously been inconsistent with the conditioning theory now became comprehensable.

\subsection{Adaptation of the classical conditioning theory}

Not all behaviourists accepted the alternative theories. As stated in section 1.4, a group of theorists, mainly experimental learning theorists, claimed that it was not necessary to add new theories to the classical conditioning theory. These neobehaviourists argued that most anomalies could be dealt with if the concept of classical conditioning itself was adapted to modern insights (see also table 1.1).

Eysenck $(1968,1976)$ developed the incubation theory of anxiety to help to explain why unreinforced presentation of the CS may lead to enhancement of the $C R$, instead of extinction. In this theory, a distinction is made between Pavlovian $A$ and $B$ conditioning; conditioning with $(B)$ and without (A) stimulus substitution. In the case of Pavlovian $B$, the unreinforced presentation of CS may not result in extinction of the $C R$, because the conditioned fear may become aversive in itself and act as a reinforcer, thus providing no extinction but an increase in the strength of the conditioned fear. The CR may become even stronger than the UCR. If a mild US/ UCR combination is the beginning of a progressively increasing $C R$, the original US will be scarcely (if ever) remembered.

The social-behaviourists argued that the indirect pathways to acquire fear could be incorporated into the classical conditioning paradigm. Staats $(1968,1975)$ showed that the paired presentation of positive/negative words with neutral stimuli led to physiological changes as well as changes in overt behaviour towards these formerly neutral stimuli. Language conditioning seemed thus to follow the same rules as traditional forms of classical conditioning. Again, this theory offered an explanation as to why anxiety disorders are not always preceded by traumatic events. According to Eifert (1987), language conditioning could explain why fears are not equally distributed: fear-relevant stimuli would have more negative strength because of previous cultural learning. In variaus experiments Hekmat (1972, 1977) investigated the therapeutic effects of language conditioning in animal phobics. Phobic word stimuli (snake, spider) were paired with positive words (vacation, joy) or positive images, resulting in a less negative affective evaluation of the phobic animal and an increase in approach behaviour.

In a series of animal studies, Kamin (1969) demonstrated that the paired presentation of stimulus and reinforcer will not automatically lead to conditioning. When these pairings occur in the presence of another well-established CS, the new conditioning will be blocked. In a typical blocking experiment, the experimental group learns in the first stage of the experiment that CS1 predicts the US, while the control group is not conditioned to CS1. In the second stage, both groups are confron. ted with a compound stimulus, a combination of CS1 and CS2, and this compound can predict the occurrence of the US. When Kamin subsequently tested conditioning to CS2 by presenting it alone, remarkable differences in strength of conditioning became apparent. Whereas the control group showed strong CRs, the experimental group showed weak conditioning effects, although the number of CS2/US pairings was the same for both groups. Kamin suggested that only surprising reinforcers can be conditioned; if the US is already well predicted by another CS, CS1 in the example given, the addition of a second CS (CS2) is superfluous in predicting the 
US, thus no conditioning occurs. Rescorla and Wagner (1972) linked up the results of these and other, related experiments and proposed an informational model of selective classical conditioning. They stated that the associative strength of a stimulus (S) depends upon the total associative strength of the compound of stimuli in which this particular stimulus appears. If this total associative strength is high, and the US is well predicted by other stimuli, reinforcement will be relatively ineffective in producing an increase in the strength of $\mathrm{S}$ (cf. Kamin blocking effect). However, if the total associative strength is high, and the presentation of $S$ is not followed by the US, the addition of $S$ to the compound of stimuli is of some value and the nonreinforcement will be effective in producing a decrease in the strength of $S$; predicting nonreinforcement, $S$ will become a safety signal. Strength of association is dependent on the predictive value of the CS. Consequently, when extra CSs or USs are randomly presented between the paired presentations of CS and US, it becomes harder to predict the occurrence of the US from the presence of the CS and the conditioning effects become weaker, although the total number of CS/US pairings are kept constant.

Expanding Rescorla and Wagner's selective conditioning theory, Davey (1989a, 1989 b) pointed out that the strength of the conditioned fear to a CS is not only determined by the degree to which this CS predicts the US. CRs can change radically as the $S$ revalues the meaning of the US, a process that can take place in the absence of the US itself. Referring to the outcomes of various experiments, Davey argued that an originally mild US can, after revaluation, backwardly initiate strong conditioned fear responses. Revaluation processes may lead to inflated CRs, but also to decreased CRs. US revaluation therefore offers an explanation for both the first and the second anomalies.

\subsection{Concluding remarks}

Regardless of the position adopted towards the anomalies in classical conditioning theory, cognitive approaches became more accepted in behaviour therapy and theory. The pragmatic clinicians used various cognitive techniques in their broad spectrum treatments, for instance by changing the 'self talk' in anxious subjects (Meichenbaum, 1975). And the conditioning theorists, either adapting the original conditioning theory or accepting alternative theories in addition to classical conditioning, incorporated more or less cognitive elements in their theories. For example, the transgression from S-R learning to S-S learning, as described in section 1.5, involved a change from reflexological learning to the learning of representations. Although conditioning theories still formed the cornerstones of behavioural theories, cognitive processes and principles were obviously taken into account. 


\section{Chapter 2}

\section{Cognitive theories and empirical data: Self-reports and information processing experiments}

The present chapter describes briefly three cognitive theories of emotional disorders, their metatheoretical perspectives and empirical data supporting and opposing these theories. The cognitive theories and their metatheoretical perspectives are introduced in the first section. The following section discusses the fact that anxious subjects report having danger-related thoughts, thereby supporting one of the basic assumptions of cognitive theories. However it is argued that these theories can not be verified on the basis of self-report data alone. Un the following sections information processing experiments are reviewed. Three stages of information processing can be distinguilshed: attention, identification and interpretation, and retrieval processes. Whereas there is ample evidence that anxious subjects pay selective attention to threatening stimuli, data concerning later stages of information processing is not so clear. Experimental findings, reviewed in section 2.5, suggest that identification and interpretation of threatening stimuli are only facilitated if competing stimuli or interpretations are available. In the subsequent section, experiments concerning the retrieval of threatening information are discussed. Results of memory experiments are confusing and do not unequivocally support the hypothesis that anxious subjects will remember selectively threatening information. The chapter concludes with some remarks on the significance of information processing experiments for cognitive theories. 


\subsection{Cognitive theories}

While the empirical work on classical conditioning steadily progressed and the attitude of behaviourists towards cognitive factors was softened, very new and different perspectives were developed in psychology; perspectives that are conveniently described as 'cognitive theories' or 'information processing theories'. They were strongly inspired by the rise of computer technology and thought of the mind as a living computer. Like a computer, the mind is supposed to process meaningless bits of sensory input in order to build up more complex units of information (Lazarus, 1981). The original information processing approach was thus silent on the influence of motivational drives in people.

As long as information processing research was concerned with 'cold' cognitions and ignored affective processes, the relevance of cognitive theories for emotional disorders was rather limited. It was mainly when cognitive theories expanded and began to deal with emotions that they became relevant for psychopathology. Whilst there was a general consensus that cognitions and emotions are in some way linked, the nature of the interrelationship was the subject of extensive debate. A crucial issue was whether all emotions are preceded by cognitive activity, like appraisal of a situation, or if emotions can be pre-cognitive. According to Zajonc, cognition and affect are likely to be under the control of two separate neuroanatomical structures (Zajonc, 1984) and affect can be aroused independently of cognitive processes. Zajonc referred to the outcomes of various experiments which showed that unfamiliar stimuli like Japanese ideographs, melodies and polygons (all reviewed and cited in Zajonc, 1980) were rated more positively if the subject was previously exposed to these stimuli, even when recognition was prevented. This evidence was dismissed by his opponents, with Lazarus (1981, 1982, 1984) as their spokesman, with the argument that all emotions are preceded by cognitive processes, but that these processes can be so rapidly that the subject can remain unaware of them. Jumping the next few sections, it can be stated that most current cognitive theories of emotional disorders in fact assume such unconscious cognitive processes. But it is hard to envisage how such an assumption may be falsified.

Basically, cognitive theories of emotional disorders comply with the following reasoning. Certain (inypothetical) mental structures are responsible for the processing of sensory information. This information processing is not unbiased but mood congruent, i.e. the sensory information is interpreted in correspondence with the prevailing mood and will preserve and strengthen the current emotional state. The various cognitive theories of emotional disorders are distinguished by the stage of processing they are focusing on, by the exactness of their definitions of the relevant structures and processes and by the metatheoretical perspective which is taken. The predominant perspective in cognitive psychology is the information processing approach (Safran \& Greenberg, 1988), relying heavily on the original theories in which emotions are considered as the final product of information processing. A second metatheory of cognitive psychology is the functional ecological approach, concerned with understanding the adaptive significance of human functioning in the context of the ecological environment. Unlike computers, human beings are held to act constantly upon their environment and to acquire information about this environment by this action (Safran \& Greenberg, 1988). From this point of view, a more interactive relationship between cognitions and emotions can be expected. Some cognitive theories, that explicitly deal with the relationship between cognition and 
emotion, are described by Beck (e.g. Beck, 1976). Bower (e.g. Bower, 1981) and Lang le.g. Lang, 1977/.

Beck's cognitive theory (Beck, 1976; Beck \& Emery, 1985) is largely based on clinical experience. It is a global theory concerning all stages of information processing: encoding, interpretation, storage and retrieval. According to Beck, individuals vulnerable to anxiety states are characterized by overactive cognitive structures, which are specifically concerned with the processing of danger-related information, the so-called 'danger schemata'. Since activation of these danger schemata will favour the encoding and storage of schema congruent information about personal danger and imposes schema congruent interpretations on ambiguous information, anxiety patients become hypersensitive to threat cues. This hypersensitivity further intensifies the anxious mood, and an anxiety disorder develops and maintains as a result of a vicious cycle of selective processing and anxious mood. Although very reasonable and useful for clinical practice, Beck"s schema theory remains vague on crucial points. For instance, it lacks precise definitions and it remains unclear as to how danger schemata are constructed and how exactly the various stages of information processing are executed. Basically, Beck's theory is an information processing theory but he also mentions, in a rather casual manner, the functional relevance of cognitive distortions for the self-protection of the subject (Beck \& Emery. 1985, p. 56).

Bower was originally interested in the issue of mood state dependent memory. Although evidence for the original hypothesis is mixed (see for reviews Blaney, 1986; Bower, 1987; Singer \& Salovey, 1988) and the theory apparently needs to be qualified, the model is still of current interest. Bower (1981, 1987) placed his work within an accepted framework of cognitive psychology, a network madel. Human memory is assumed to be organized in an associative network, in which each event is represented as a cluster of connected descriptive propositions or nodes. He supposed that emotions are specific nodes, embedded in a network of relevant memories, representations of concepts and actions. Activation of one node in the associative network will result in a spreading of activation through which the associated nodes are triggered; the stronger the associations the more the connected nodes will become activated. Like Beck's theory, the associative network theory predicts mood congruent information processing in all stages of information processing, although in Bower's work emphasis is put on the later stages of processing (storage and memory). The model is more elaborate than the schema theory and typically formulated from a nonclinical information processing point of view.

In his bio-informational theory, Lang $(1977,1979,1984,1985)$ also started from a network approach to emotions. According to Lang, fear and other emotions are represented as separate networks in memory that include information about stimulus characteristics, response patterns and the meaning of stimuli and responses. Lang considered a fear network as a programme for escape and avoidance, and argued that interpretations about the meaning of stimulus and response elements are important components in the network. As Foa and Kozak (1986, p.21) stated: "The programs for running ahead of a baton-carrying competitor in a race and for running ahead of a club-carrying assailant on a racetrack are likely to involve similar stimulus and response information. That which distinguishes the fear structure is the meaning of the stimuli and responses". Although a network model, Lang's theory offers an ecological approach to cognitive psychology. 


\subsection{Self-report data}

Inspired by cognitive theories, several clinical studies were carried out in order to investigate the thought contents of anxious subjects. By means of structured interviews, anxious subjects were asked about their thoughts during periods of increased anxilety. Results of these studies confirmed the cognitive view. All generalized anxiety patients in a study by Beck, Laude and Bohnert (1974) reported thought contents concerning personal danger, either physically or socially. Beck and Rush (1975) found different effects for phobic and generalized anxiety patients: thoughts reported by phobics were restricted to relatively specific external sources of danger and were less often concerned with internal bodily sensations. A decade later, Hibbert (1984) found comparable results in anxiety-state patients. They reported danger-related thoughts and after dividing them in two sub-groups (with and without panic attacks). Hibbert noted that panic patients were more likely to report catastrophic thoughts, related to sudden death or severe disease, than non-panic patients. Such thoughts are however not exclusively found in panic patients; Arntz, Lavy, van den Berg and van Rijsoort (1993b), using an irrational bellefs questionnaire, showed that spider phobics frequently held panic-related beliefs, like the belief that confrontation with the spider could cause a heart attack or loss of contral.

Although suggestive, the results of these studies are not convincing because of serious methodological problems. Self-report techniques rely heavily upon the introspective abilities of the subjects. Nisbett and Wilson (1977) showed in an extensive enumeration of experimental and anecdotal data that verbal reports of cognitive processes are often unreliable. They argued that subjects may be unaware of a stimulus that brought an important influence to bear on their responses, and may even be unaware of showing any (change in) response at all. Besides, even if the subjects are aware of both stimulus and response, they often appear not to realize that the stimulus has affected the response. Nisbett and Wilson (1977) suggested that verbal reports of cognitive processes are based on a priori causal theories. In reply to their exposition of facts, it is argued that inaccurate reports of cognitive processes are insufficient evidence on which to assume that subjects are unaware of their cognitive processes (e.g. Williams, Watts, MacLeod \& Mathews, 1988 . But the two alternative explanations that are offered, failure of memory or difficulties in verbalizing the conscious experience, are equally devastating arguments against the reliability of self-report techniques.

The self-report studies on cognitions in anxious subjects suffer from further methodological problems. Since most of the studies did not incorporate nonanxious control groups, it is unclear whether the results were indeed specific for anxiety patients. Only Arntz et al. (1993b) compared anxious, spider phobic, and nonanxious control subjects. Discriminating between spider-related beliefs and selfrelated beliefs, they found strongly significant differences between phobics and controls on all subscales. A third problem, more difficult to get round, is the transparency of the method used. It is not too difficult for subjects to guess the aim of the thought content studies and therefore, demand or expectancy effects cian not be ruled out, not even in a study including nonanxious controls. Finally, even if there was a way of solving all these problems and if it were found that anxiety patients report more danger-related thoughts than nonanxious controls, the qustion would still remain as to whether this difference in thought content was the cause of 
the anxiety disorder, or whether it was merely one of its consequences. In sum, self-report studies are insufficient to verify cognitive theories.

\subsection{Experimental data}

Behaviourism evolved as a reaction to intrapsychic oriented theories, and held as crucial the tenet that a scientific psychology could and should be concerned exclusively with observable behaviour. It was therefore no surprise that the return of interest in non-observable mental processes, as upheld by cognitive theories, met with considerable sceptism from behaviouristic advocates (e.g. Eysenck, 1987). But, of course, postulating non-observable processes is not anti-scientific in itself, the crucial question is whether the postulated processes can be linked to empirical data so that the existence and nature of these processes can be corroborated or falsified.

In general cognitive psychology, several experimental tasks were developed to investigate how information processing normally takes place and how it is phased. Examples of these laboratory tasks, which typically did not rely on self-reports, are Stroop colour naming tasks, subliminal perception tasks, lexical decision tasks, recall and recognition tasks etc. Initially, these tasks included only non-emotional stimuli, such as neutral words, meaningless letter strings or geometric figures. When emotions were included in the field of cognitive psychology, cognitive researchers became interested in how 'ordinary' information processing is in fluenced by the emotional valence of that information.

By manipulating the emotional valence of stimuli, the influence on separate stages of processing can be investigated. It is found that subliminal or dichotic presentation of threat-related stimuli results in an increase of anxiety le.g. KempWheeler \& Hill, 1987) and skin conductance responses respectively (Corteen \& Wood, 1972), in spite of the subjects' unawareness of the stimuli presented. In several experiments the allocation of attentional resources was investigated, showing that normal subjects pay more attention to undesirable information (Pratto \& John, 1991) and taboo words (Nielsen \& Sarason, 1981) than to desirable or neutral stimuli. Bradley (1993), reviewing several experiments concerning emotional memory, concluded that the emotional valence of stimuli was less important than the level of arousal: both free recall and speed of recognition were better if the stimuli were highly arousing, whilst evidence for the influence of emotional valence was mixed, with a slight preference in normal subjects to show better memory for pleasant than for unpleasant stimuli. In general it can be concluded that various stages of information processing in normal subjects are influenced by the emotional saliency of that information, but the separate roles of emotional valence and arousal have to be clarified.

A second, and more important, line in the research of emotional processing investigates interactions between mood states at encoding and retrieval (mood state dependency) or interactions between mood states and emotional valence (mood congruencyl. Typically, two mood states, happiness and sadness, are investigated. Happy and sad moods are induced in normal subjects by use of hypnosis, reading emotional self-statements (the 'Velten's procedure') or listening to music with a strong affective impact. Mood congruency research investigates whether subjects tend to detect, learn and recall information better when that information is congru- 
ent with their mood. Mood state dependency research looks at whether items, learned in a mood state that is similar to the mood state at retrieval, are remembered better. Whilst evidence for mood state dependent recall is mixed (see also section 2.11, mood congruent learning and recall are frequently confirmed (see for reviews Blaney, 1986; Singer \& Salovey, 1988). Studies concerning the mood congruent detection are scarse and findings are contradictory 1 Gerrig \& Bower, 1982. Small \& Robins, 19881. In this context the results of several priming studies are relevant; both in lexical decision tasks (Hill \& Kemp-Wheeler, 1989a; KempWheeler \& Hill, 1992) and evaluative decision tasks (Greenwald, Klinger, \& Liu, 1989 ) it is found that response is facilitated if the target is preceded by a congruent, but undetected, prime.

The influence of emotionally valenced information may also be studied in emotionally disordered subjects. From the models by Beck, Bower and Lang similar predictions about mood congruency effects can be gained. Cognition \& emotion experiments can therefore not be used to elucidate the relative merits of specific models. All models assume, explicitly or implicitly, that anxious subjects show pervasive mood congruent cognitive biases, favouring anxiety-relevant information at all stages of information processing; demonstration of differences in the performance of anxious and nonanxious subjects can therefore be held to support any of the cognitive theories:

The processing of anxiety-relevant and anxiety-irrelevant information in anxious subjects has been investigated in numerous experiments. These studies can be categorized by the stage of processing they focused on, resulting in a set of studies relating to attentional processes, processes related to the identification and interpretation of information, and retrieval processes. The evidence will be reviewed in this order.

\subsection{Selective processing in anxiety states: Attentional processes}

The allocation of attention is the first stage in information processing that has been investigated in relation to anxiety states. The assignment of attentional resources is a crucial operation, since it may determine which information is to be processed further. Cognitive theories imply that anxious subjects will pay relatively more attention to anxiety-relevant information, i.e. from the bulk of information that is available in natural circumstances they selectively attend to stimuli that are related to threat and danger.

It should be noted that selective attention cannot be established by measuring the attention that subjects pay to an isolated stimulus. In that case, it is not selective attention that is measured but the speed of identification (see section 2.5). The hypothesized attentional bias in anxious subjects can thus only be shown if there are other stimuli which could also be paid attention to. Attentional processing in anxious and normal subjects has been investigated in numerous studies. As the present dissertation focuses on attentional processes, a separate chapter (chapter 3) has been devoted to this subject. Several paradigms are used, which can all be considered as being variations on one common principle. Subjects carry out some cognitive task and concurrent threatening and/or neutral information is presented at the same time or shortly before. Because attentional resources are limited, the distraction produced by the concurrent threatening/neutral information on the primary 
cognitive task is an indication of the allocation of attientional resources; if the primary cognitive task requires that the concurrent information is ignored, performance on the primary cognitive task will deteriorate the moment the concurrent information demands attention. And if threatening concurrent information results in a stronger deterioration than concurrent neutral information, it can be concluded that the subject selectively attends to threatening stimuli. On the other hand, if attention can be focused on the concurrent information, selective attention to threatening concurrent information will result in a facilitation of response to the primary attention task. Normal and anxious subjects can be compared on both types of attentional deployment tasks, thus making possible the investigation of group differences in the allocation of attention.

There is already ample evidence that anxious subjects indeed show an atterytional bias to threat-related information, even when they are explicitly instructed to ignore this information and even if they seem to be unaware of their preference. In chapter 3 , the subject of attentional bias and interesting related topics are reviewed and discussed more extensively.

\subsection{Selective processing in anxiety states: Identification and interpretation}

One of the assumptions of cognitive theories is that mood congruent (i.e. thireat related) representations are enhanced accessible in anxious subjects, and this assumption leads to some straightforward predictions. The first prediction is that anxious subjects can identify threatening stimuli more quickly than nonthreatening stimuli. Consequently it can be predicted that, if two concurring interpretations are possible, the more threatening interpretation will come more readily in mind and will be judged as more likely than the nonthreatening interpretation: Cognitive researchers have used a range of, sometimes highly inventive, experimental paradigms to investigate these predictions.

While the first prediction, that anxious subjects can identify threatening stimuli more quickly, is more directly connected with the assumption of cognitive theories, traditionally the focus of research has been on the interpretation of ambiguous stimuli. Butler and Mathews [1983) asked generally anxious, depressive and normal subjects to read ten ambiguous scenarios, which could be interpreted as threatening le.g. "You wake with a start in the middle of the night, thinking you heard a noise, but all is quiet"). Subjects were asked to give the first explanation that came to mind, and then to arrange three given explanations in the order in which they would be most likely to come to mind if the subject was in that situation. From the three alternatives, only one was threatening. Although there was a trend in the expected direction, no significant differences were found on the first, open-ended question. However, both anxious and depressive subjects judged the threatening alternatives as more likely to come to mind than did the normal control subjects. Since the depressive subjects proved also to be anxious, this finding did not conflict with the hypothesis. McNally and Foa (1987) replicated this study with treated and untreated agoraphobics and hypothesized that they would be more likely than normals to give a threatening interpretation to ambiguous internal, but not to ambiguous external information. Untreated agoraphobics gave more threatening interpretations to the open-ended question and ranked the threatening alternatives as more likely than did either the treated agoraphobics or the normal controls. But there was 
no evidence that this interpretative bias was exclusively towards internal information; agoraphobics showed a general tendency to interpret ambiguous information as threatening. Both the Butler and Mathews (1983) and the Foa and McNally (1987) studies thus provide some support for the prediction that anxious subjects tend to interpret ambiguous information in a threatening way. The rank-ordering of experimenter-provided alternatiwes yielded the most consistent results; in comparison with nonanxious subjects, anxious subjects said that they thought the most threatening explanation would be more likely to come to mind if they were in that particular situation. It can however be questioned whether people can make such judgements correctly and whether the judgements are well-grounded; as discussed in section 2.2 introspective capacities often prove to be weak, and frequently subjects make judgements on the basis of a priori theories instead of on true insight in current processes.

Eysenck, MacLeod and Mathews (1987a) used a more sophisticated paradigm to elucidate the question of interpretative processes. They presented homophones, words which have both threatening and nonthreatening meanings but different spellings for each meaning (such as "guilt' and "gilt"/"die" and 'dye") to a group of normal subjects and asked these subjects to write down the list of words they heard. Eysenck et al. (1987a) found a significant association between the number of threatening spellings written down, and the level of trait anxiety. In a successive study Mathews, Richards and Eysenck (1989b) presented a list of homophones to three groups of subjects: untreated and treated subjects with general anxiety disorder and normal controls. The results showed that clinically anxious subjects used the threatening spelling relatively more often than did controls, whereas recovered subjects took an intermediate position.

Two years later Eysenck, Mogg, May, Richards and Mathews (1991) argued that the preference anxious subjects showed for the more threatening spelling could not be taken as a prove that they were unaware of the alternative, nonthreatening interpretation. Subjects may be consciously aware of both interpretations and, forced to write down only a single spelling, may have been influenced by demand characteristics in choosing a spelling. Besides, Eysenck et al. (1991) stated that the homophone paradigm is not a naturalistic procedure, since in normal life words do not occur in isolation but within a semantic context. In two experiments they presented audio-taped ambiguous sentences to generally anxious and control subjects. Afterwards subjects were shown a list of unambiguous sentences and they had to decide whether each of these sentences had (more or less) the same meaning as a sentence heard earlier on the tape. Currently anxious subjects were more likely than normal controls and recovered anxious subjects to interpret the ambiguious sentences in a threatening way. Moreover, Eysenck et al. (1991) showed that this interpretative bias was not due to response bias. Strictly speaking, it is impossible to decide whether the Eysenck et al. (1991) findings reflect an interpretative bias or a memory bias. The possibility remains open that a subject is aware of both interpretations when the audio-tape is played, but afterwards selectively remembers only one interpretation. The results which were attributed to a bias in interpretation, could thus also result from a memory bias.

The sentence completion task used by Stoler and McNally (1991) did not circumvent this explanatory problem. They presented subjects with sentence stems which were either ambiguous or unambiguous, and had either a potentially threatening or neutral meaning. Subjects were asked to complete each stem with the first thought 
that came to mind. Both symptomatic and recovered agoraphobics completed more potentially threatening ambiguous stems in a threatening way than did normals.

Overall, results of the ambiguous information studies discussed in this chapter provide firm evidence for an interpretation bias in anxious subjects. That is, threatening interpretations seem to come quicker to mind than nonthreatening explanations and are also judged to be more likely.

In contrast to these supportive findings, several failures to confirm the first prediction, that anxious subjects can identify threatening information more quickly, have been reported (summarized in MacLeod, 1990; MacLeod \& Mathews, 1991: Mathews, 1988). For example, anxious and normal subjects did not differ in their tachistoscopic recognition thresholds of threat-related and neutral stimuli. Nor did they differ in response times to threat-related words, presented in a lexical decision task. Hill and Kemp-Wheeler $(1989 \mathrm{~b})$, testing high and low trait anxious subjects, also found that the lexical and affective decision times of high anxious subjects were comparable for both threatening and pleasant stimuli.

Thus, although the results of the ambiguous information studies suggest that threatening information is more easily and more quickly identified than nonthreatening information, these biases appear to stay undetected when subjects are presented with single and unambiguous stimuli. In line with these findings, MacLeod and Mathews (1991) suggested that anxiety is associated with the assignment of high processing priorities to threat-related alternatives, rather than with the facilitated avallability of threat-related information in memory. In order to test this hypothesis, they presented generaly anxious and control subjects with a lexical decision task, including both single and double string displays. Subjects had to decide as rapidly as possible whether or not a ward was presented on a VDU screen. If anxiety enhances the availability of threat-related representations, anxious subjects would show more rapid responses to threat words, on both the single and the double string displays. But, if anxiety biases the assignment of processing priorities to threatening information, then anxious subjects would only show speeded lexical decision latencies for threat words under double string conditions, when priorization is required. MacLeod and Mathews (1991) found that anxious subjects only showed facilitated processing of threat-related material under double string conditions, giving support to the hypothesis that anxious subjects, in comparison to normal subjects, give priority to the processing of threat-related information. It is notable that in general, the anxious subjects had more delayed lexical decision latencies than did the normal controls; the facilitated processing of threat-related stimuli in anxious subjects was therefore relative to the neutral stimuli. Mogg, Mathews, Evsenck and May (1991a) carried out a replication study, but their results were only partially consistent with the MacLeod and Mathews (1991) findings. Their anxious subjects showed only an anxiety-related lexical decision bias if there was both competition for processing priorities (double string condition) and the anxiety-related information was presented outside the focus of the subject's attention.

In sum, there is some evidence to suggest that the facilitated processing of threat-related material in anxious subjects is not due to the enhanced availability of this material from memory, but to preferential processing of potentially threatening information when two competing interpretations or options are available. Since complex and ambiguous situations are part of daily life, probably outnumbering the simple and straightforward situations, the preferred processing of potentially threatening information in anxious subjects results in a heightened probability of perceived 
danger (see also Mathews, 1988). Priorization of processing is therefore not necessarfy in opposition to a network or schema model of anxiety disorders, but indicates under what conditions the mood congruent interpretative biases may or may not occur.

\subsection{Selective processing in anxiety states: Retrieval processes}

In depression research, there is a long tradition of investigating the effect of depressive mood states on memory capacities (for reviews see Blaney, 1986; Singer \& Salovey, 1988; Williams et $a /, 1988)$. The general conclusion is that depressive subjects, compared with nondepressive subjects, more rapidly retrieve unpleasant than pleasant personal memories and tend to selectively recall negative stimuli after learning several word lists. From cognitive theories, similar biases in the retrieval of mood congruent information would be expected in anxious subjects.

Whereas research on mood and memory in depression has resulted in relatively firm conclusions, the relationship between anxious mood and memory is still unclear. Relevant characteristics and results of mood and memory experiments in anxiaty states are summarized in table 2.1. In these studies two memory tasks are frequently used, namely recall and recognition tasks. In a typical experiment anxious and normal subjects are presented with audio-taped word lists, including both threatening and nonthreatening stimuli. Subjects are instructed to listen to the tape and afterwards to write down all the words they remember (recall), or to mark on an inventory which words were on the tape and which were not (recognition). The results obtained with recall or recognition tests vary from a memory bias in favour of threatening stimuli (Cloitre \& Liebowitz, 1991; McNally, Foa, \& Donnell, 1989; Watts \& Coyle, 1992; Zeitlin \& McNally, 1991), to a memory bias at the expense of threatening information (Foa, McNally, \& Murdock, 1989a; Mogg, Mathews, \& Weinman, 1987; Watts \& Dalgleish, experiment 1, 19911, to mixed results depending on the task (Watts \& Dalgleish, experiment 2, 1991), and finally to no memory bias at all (Mathews, Mogg, May, \& Eysenck, 1989a; Mogg \& Mathews, 1990 ).

Recall and recognition tasks are regularly applied to attentional research with the expectation that stimuli that are selectively attended to, will also be better remem. bered. This prediction was never confirmed (Beck , Stanley, Averill, Baldwin, \& Deagle, 1992; Ehlers, Margraf, Davies, \& Roth, 1988; Mathews \& MacLeod, 1985; Mathews \& MacLeod, 1986; Mogg, Mathews, \& Weinman, 1989; Richards \& Millwood, 1989). Despite these negative results Arnold, Hartley and MacLeod (cited in MacLeod, 1990) suggested that, since in attentional and interpretative processing, biases are only demonstrated when alternative processing options are present, the need to prioritize may also be crucial for memory bias to occur. They conducted an experiment to investigate the differences between divided and focused attention conditions. Subjects were presented with pairs of words, appearing on two separate screens. In one condition words were presented sequentially and in the other condition simultaneously, the latter requiring that the subjects assigned their own priorities to the processing of each word. Measuring recall latency after each trial, they found no differences between high and low trait anxious subjects in the focused attention condition. Neither in the divided attention condition did high trait anxious subjects show a memory bias in favour of threatening words; on the contrary, their recall latencies for threatening words were longer than for neutral words. 


\begin{tabular}{|c|c|c|c|c|c|c|c|c|c|}
\hline 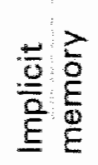 & & ' & 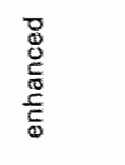 & ' & . & ' & & 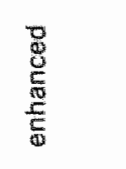 & \\
\hline 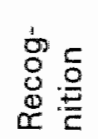 & & 惫 & & 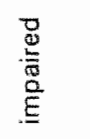 & & 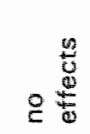 & 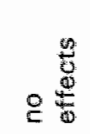 & & \\
\hline $\begin{array}{l}\overline{\bar{\sigma}} \\
\mathbb{8}\end{array}$ & 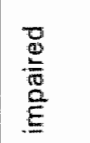 & 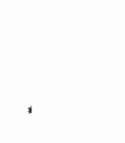 & 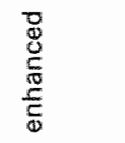 & , & 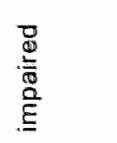 & ' & & 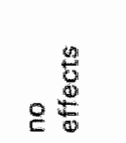 & 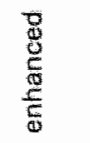 \\
\hline 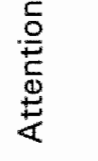 & 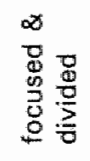 & 总 & 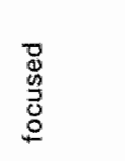 & 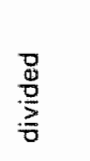 & 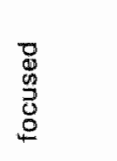 & $\begin{array}{l}\frac{\mathrm{g}}{\mathrm{g}} \\
\frac{\mathrm{g}}{\mathrm{z}}\end{array}$ & 总 & 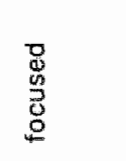 & 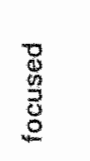 \\
\hline $\overrightarrow{\underline{E}}$ & 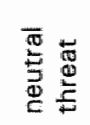 & 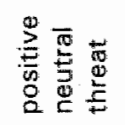 & 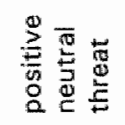 & 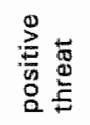 & 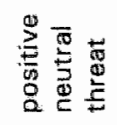 & 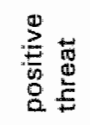 & 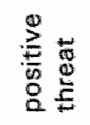 & 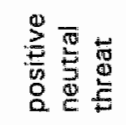 & 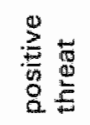 \\
\hline$\ddot{~}$ & $\begin{array}{l}\frac{4}{8} \\
\frac{0}{3}\end{array}$ & 哭 & $\frac{n}{\frac{0}{0}}$ & $\begin{array}{l}\frac{n}{2} \\
\frac{0}{3}\end{array}$ & 照 & $\frac{n}{50}$ & 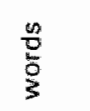 & $\begin{array}{l}0 \\
:\end{array}$ & $\frac{\infty}{\square}$ \\
\hline $\begin{array}{l}\frac{0}{x} \\
\frac{1}{x}{ }^{\circ}\end{array}$ & 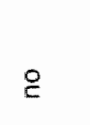 & $\stackrel{\circ}{c}$ & \& & $\stackrel{\circ}{\varepsilon}$ & 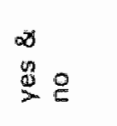 & $\cong$ & $q$ & $\stackrel{8}{\circ}$ & $\frac{9}{2}$ \\
\hline$\frac{\pi}{0}$ & 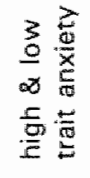 & 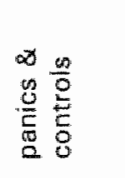 & 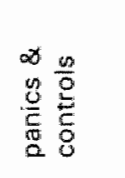 & 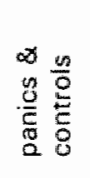 & 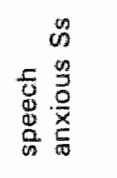 & 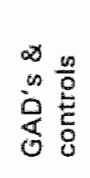 & 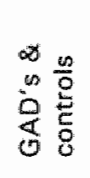 & 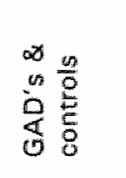 & 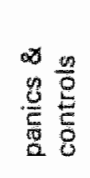 \\
\hline 는 & 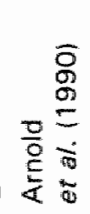 & 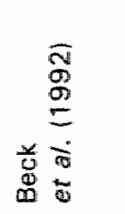 & 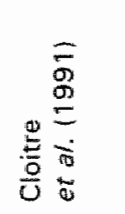 & 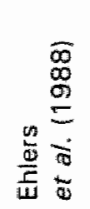 & 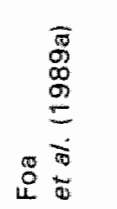 & 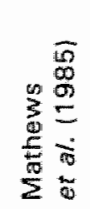 & 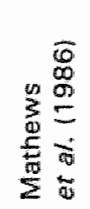 & 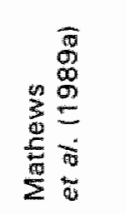 & 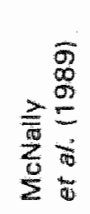 \\
\hline
\end{tabular}




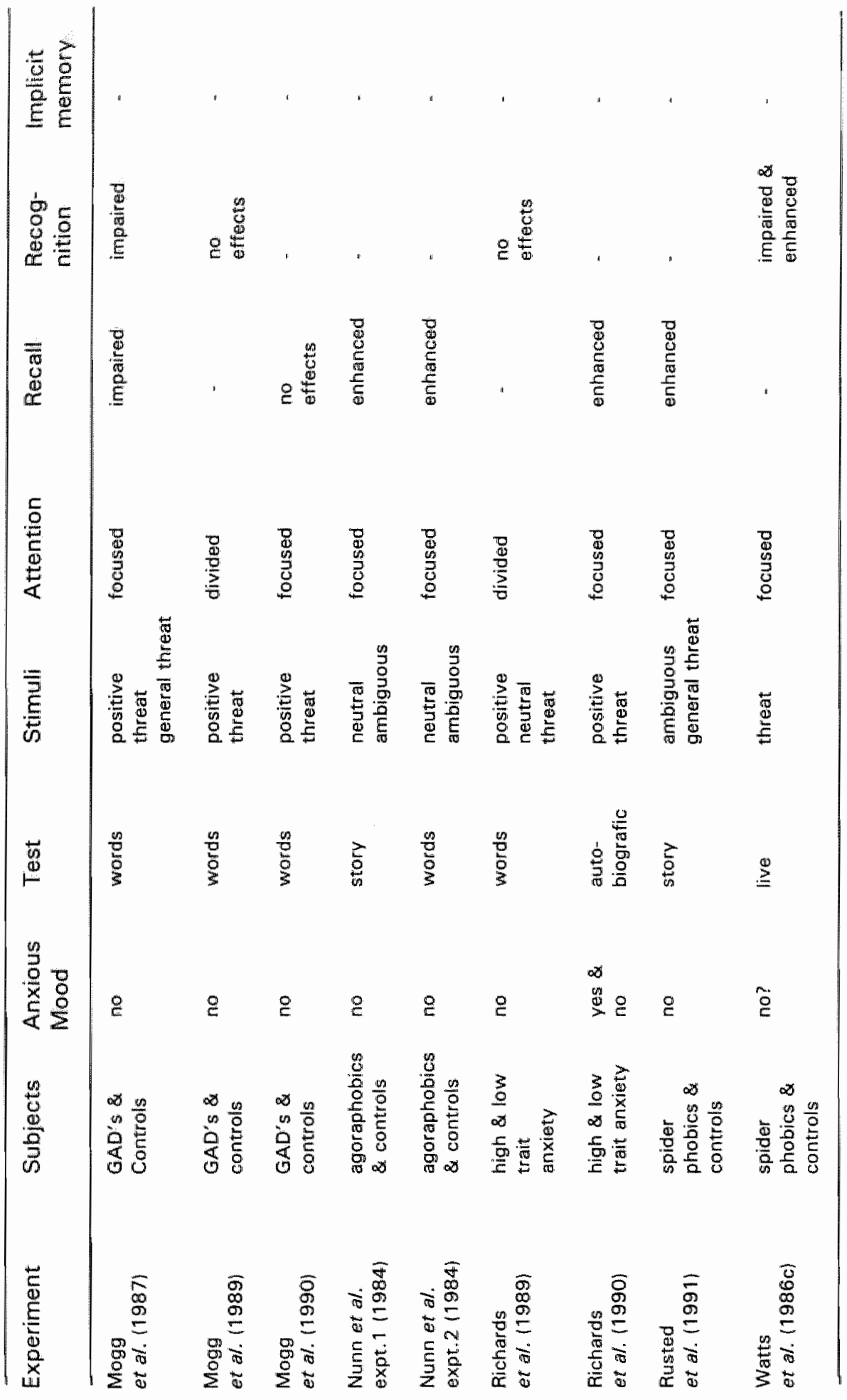




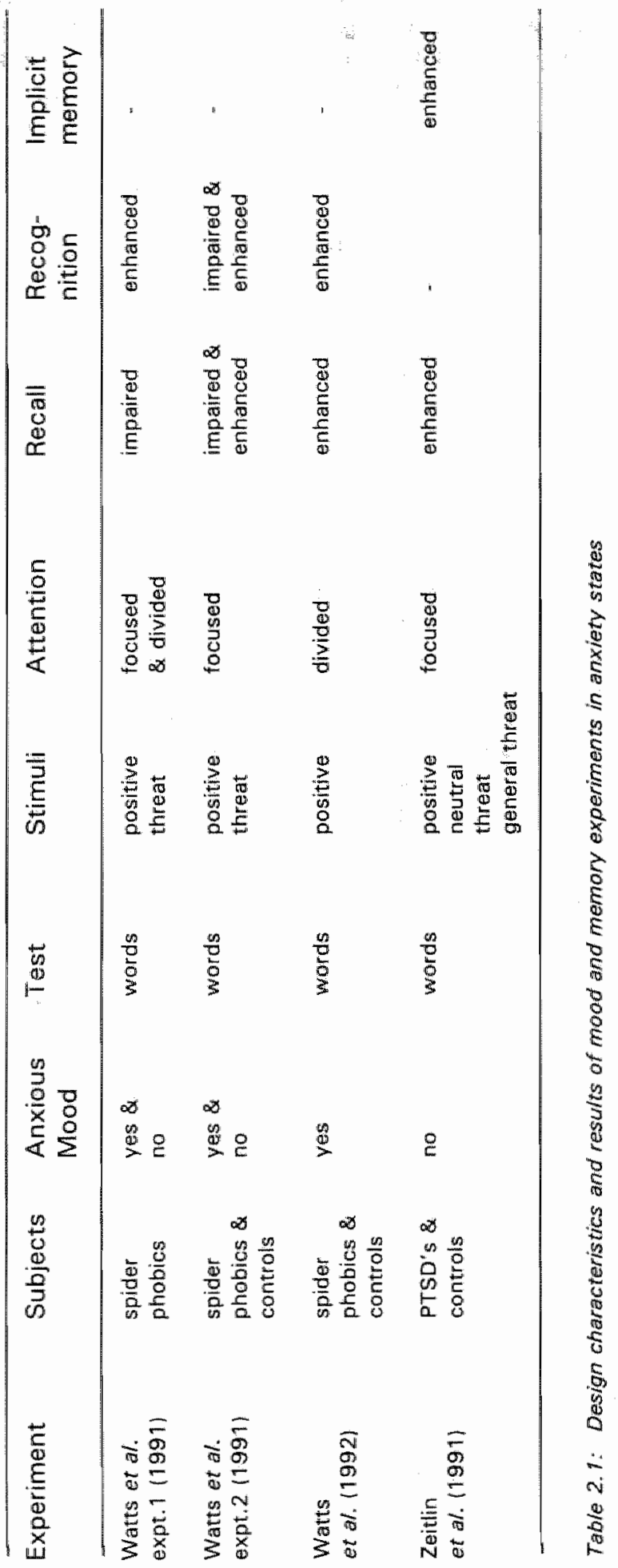


To summarize the results of the various studies with words or word lists, evidence for memory bias is confusing. Under focused attention conditions results are contradicting; from these studies it remains unclear whether anxious subjects show any memory bias at all, and if so, whether this bias would be in favour or at the expense of threatening information. There is only one study as yet, in which retrieval processing is directly measured under divided attention conditions, providing some evidience for impaired memory of threatening information in anxious subjects. But the memory tests applied to attentional bias experiments may also be considered as measuring memory performance under divided attention conditions, and in these tests no evidence was found for any memory bias whatsoever.

Although stimulus words are dominant in memory research, several studies have made use of alternative stimuli which seem to be more natural, such as stories, live stimulli or personal memories. Watts, Trezise and Sharrock (1986c) exposed spider phobics and controls to freeze-dried, but real spiders and found that spider phobics tended to recognize less big spiders than nonanxious controls, whereas the opposite was true for small spiders. Richards and Whittaker (1990), following the studies focusing on depressive mood states, asked high and low trait anxious subjects to retrieve as quickly as possible a personal memory on the basis of several threatening and pleasant cue words. Within each group, half of the subjects were primed with unpleasant pictures and half were not. Richards and Whittaker (1990) found that priming did not affect high trait anxious subjects, that is, under both conditions, they showed shorter retrieval latencies for anxiety-related memories than for happiness-related memories. But the low trait anxious subjects only showed such memory bias towards unpleasant memories if they were initially primed with unpleasant pictures. In several experiments stories were used as stimuli (Nunn \& Whalan, experiment 1, 1984, Rusted \& Dighton, 1991) but, in contrast with stimuli used in other memory experiments, these stories were not unambiguously threatening. For example in the Nunn and Whalan (experiment 1, 1984) study, subjects were presented with a story about "Nancy shopping for milk". And in the Rusted and Dighton (1991) study, the story included several propositions "..... which were related in some way to the presence of spiders. Spiders were not, however, mentioned explicitly at any point in the text" (p.125). It is doubtful whether nonanxious controls do recognize such stories as potentially threatening. From these narrative studies it can therefore not be concluded that anxious subjects show better recall of threatening information than nonanxious contrals, simply because these controls were not presented with threatening information.

There are markable differences in the methodological approaches of studies concerning memory processes in anxiety states. Although some of these studies are difficult to interpret, the conflicting results cannot entirely be attributed to methodological problems. As can be seen in table 2.1, several design characteristics, such as the focus of attention, the emotional valence of control stimuli or the presentation form of stimuli, appear to be of no critical interest. A potentially promising proposition is that subjects only show anxiety congruent memory biases when they are actually in an anxious mood.

Populations that have been under investigation for current mood congruency effects are speech anxious students (Foa, McNally, \& Murdock, 1989a), panic disorder patients (McNally et al., 1989) and spider phobics (Watts \& Coyle, 1992; Watts \& Dalgleish, 1991). Foa et al. (1989a) reported no evidence for mood congruent encoding effects , but they found an impaired recall for threatening words in 
subjects whose heart rate accelerated between encoding and retrieval. Since this evidence is obtained from post-hoc analyses, the results have yet to be confirmed. McNally et al. (1989) instructed panic disordered and control subjects either to relax or to take physical exercise in a five-minute period between encoding and retrieval. They did not find significant differences due to arousal effects. But self-reported anxiety did not increase in panic patients who exercised, leaving the possibility that the physiological similarity with an anxious mood state is is not enough to produce mood congruent retrieval effects.

Watts and his co-workers (Watts \& Coyle, 1992; Watts \& Dalgleish, experiment 1 \& 2,1991) manipulated the mood of spider phobic and control subjects in various ways. Watts and Coyle (1992) exposed both phobic and control subjects to a live spider during encoding, a taped recordered word presentation. Their finding that spider phobics selectively remembered spider related words is difficult to interpret, since this memory bias could be entirely attributed to the marked impairment spider phobics showed to control (i.e. baby related) words. Moreover, the influence of anxious mood during encoding could not be tested properly, since Watts and Coyle failed to add two similar groups in a nonanxious mood state. In the Watts and Dalgleish (1991) experiments, both mood congruent encoding and retrieval effects were investigated. During the encoding period in their first experiment, half the spider phobics were exposed to a live spider and half were asked to relax; the allocation to the 'exposure' and 'relax" condition during retrieval was counterbalanced. No evidence was found for mood congruent encoding or retrieval effects. In their second experiment, Watts and Dalgleish lexperiment 2, 1991) exposed spider phobics and controls, prior to encoding, to either a live or a dead spider which, albeit covered up, remained present throughout the experiment. Compared with nonanxious controls, the recall and recognition of spider related words was impaired for spider phobics in the live spider condition, but facilitated for spider phobics in the dead spider condition. These findings suggest that a small increase in anxiety facilitates the retrieval of threatening information, but that a serious increase in anxiety results in an impairment of the retrieval processes. The finding of Watts et al. (1986c) that spider phobics, compared to nonanxious controls, recognized more small spiders but fewer big spiders is consistent with this proposition.

All in all, there are no indications that heightened anxiety during encoding or retrieval simply results in a facilitated retrieval of threatening information; if anything, the relationship between anxiety and memory of threatening information seems to be curved, with optimal retrieval effects in mild anxiety but impairment in high anxiety.

One hypothesis, in line with these findings and frequently put forward $10 . \mathrm{g}$. Mathews \& Eysenck, 1987; Mathews et al., 1989a; Mogg et al., 1987), is that anxious subjects are characterized by a vigilance-avoidance pattern of cognitive processing. Anxious subjects are supposed to be on the alert against possible danger, and by continuously scanning their environment they detect and identify poten tial threatening stimuli as quickly as they can. According to the vigilance-avoidance hypothesis this rapid identification is not followed by extensive elaboration, but by cognitive avoidance of the potentially threatening information. There is a ring of intuitive plausibility to this claim; elaborating threatening information is an aversive endeavour and people are mostly inclined to abstain from activities that may produce unpleasant effects. 
Consequently Mathews et al. (1989a) made a distinction between implicit and explicit memory tasks; whereas explicit memory tests are based on remembrance of the stimuli, implicit memory tests measure the extent to which the stored information affects subsequent responses, although this information may not be remembered consciously. Mathews et al. (1989a) argued that current memory research has focused on explicit memory tests and that cognitive strategies of anxious subjects, such as avoidance and the consequent lack of elaboration, may obscure or even reverse enhanced memory of threatening information. Since implicit memory is held to involve only automatic activation and integration, thereby simply increasing the availability of the representation, Mathews et al. (1989a) suggested that implicit memory tests may be less sensitive to secondary strategic responses. The Mathews et al. (1989a) hypothesis that a memory bias in favour of threatening information can be better demonstrated by using an implicit memory task (such as word completion), than an explicit memory task (such as recall), was experimentally confirmed. After the presentation of nonthreatening and threatening words on a video screen, generalized anxiety patients did not recall significantly more of the primed threatening words, but they produced more threat word completions than did normals. This could be taken as evidence for an implicit memory bias, since for unprimed threatening words, anxious and control subjects did not made significantly different word completions. Zeitlin and McNally (1991) carried out a similar experiment with Vietnam combat veterans with and without post-traumatic stress disorder. They found an implicit memory bias for combat words only in the PTSD group, but on the recall test PTSD patients did not recall more combat words than did controls, and both groups recalled more combat words than other words. However, PTSD patients showed a relative explicit memory bias in favour of combat words; i.e. compared with the control combat veterans PTSD patients were strongly impaired in the recall of all words, except combat words. Since the PTSD subjects appeared to apply most of the available cognitive resources to combat words, this finding can be interpreted as a memory bias in favour of threatening information. Cloitre and Liebowitz (1991), using a high-speed recognition task for measuring perceptual (i.e.'implicit") memory, also found that panic patients showed a memory bias on both the recall and the high-speed recognition test. Thus, although the Mathews et al. (1989a) findings are promising and theoretically interesting, it is not entirely certain that memory biases in anxious subjects can be better demonstrated by using implicit memory tasks than by using explicit memory tasks.

\subsection{Concluding remarks}

Cognitive theories all presume extensive mood congruent cognitive biases. Anxious subjects" self reports seem to confirm grosso modo that anxious subjects have danger-related thoughts. Experimental investigations are focused on single stages of information processing in anxiety states. Depending on the stage of information processing, the experimental support for cognitive theories differs considerably. In this chapter "experimental emphasis was on the stages of identification and interpretation, and retrieval processes.

There is convincing evidence that anxious subjects do indeed interpret ambiguous stimuli in a threatening way. In anxious subjects the threatening interpretations seem to come more quickly to mind and are also judged to be more likely than by 
nonanxious subjects. The experiments concerning the identification of potentially threatening stimuli are less conclusive, but there is some evidence to believe that the identification of threatening stimuli is only facilitated if there are competing stimuli to identify. Although cognitive theories do not presume that the cognitive distortions in anxious subjects are restricted to complex situations that require priorization of processing, the experimental findings concerning identification and interpretation could still fit in the cognitive model.

Reviewing the field of memory research in anxiety states "the main conclusion is that the results are confusing and sometimes conflicting. Evidence varies from a memory bias in favour of threatening information to a memory bias at the expense of threatening information. Several explanations, like the focus of attention and the emotionall valence of control stimuli do not seem to be of crucial importance. As far as the suggested mood congruency effects are concerned, onlly mild current anxiety seems to result in the expected enhanced retrieval effects, whereas in severe current anxiety the retrieval of threatening words seems to be impaired. These findings are difficult to explain within the scope of traditional cognitive theories of anxiety disorders. A vigilance-avoidance model of cognitive processing may offer a more satisfying explanation. 


$$
\begin{aligned}
& \therefore \quad \therefore \\
& \therefore \quad \therefore \\
& \because \quad
\end{aligned}
$$




\section{Chapter 3}

\section{Attentional bias: Topics of interest}

Selective attention is the central theme of six out of seven experiments, designed and carried out by the present author and described in chapter 5 . The present chapter is therefore devoted to a review of the literature relating to attentional processing in anxiety states. The first section presents a brief discussion of the relationship between anxiety, attention and performance. The following sections focus on the phenomenon of attentional bias.

An overview is given of the paradigms used to measure attentional bias and the (clinical) populations that are presented with attentional bias tests. It is illustrated that selective attention is not unique to clinically anxious subjects. As a matter of fact, attentional biases have been demonstrated in subjects with various anxietyrelated, and perhaps even -unrelated, mood states.

In the subsequent sections, six topics are highlightened that may be rellevant for the understanding and explanation of attentional bias. Three of these topics are frequently investigated: the role of enduring personality factors (particularly trait anxietyl. familiarity of the subjects with the threatening stimuli and specificity of the emotional stimuli. The (causal) role of these issues is first critically reviewed. In the three subsequent sections topics are discussed that are regularly brought to the fore, but are rarely or never investigated: the lack of memory bias as a result of secondary avoidance strategies, the potential significance of attentional bias and the ecological validity of attentional bias tests.

Chapter 3 concludes with a summary of facts and gaps in what is known of attentional bias. 


\subsection{Attention, anxiety and performance: Introductory considerations}

In studies into the effects of anxiety on general cognitive tasks, it is frequently found that, as anxiety increases, task performance becomes progressively impaired (for reviews of literature see Eysenck, 1984; Eysenck, 1988; Eysenck \& Eysenck, 1985). Both state and trait anxiety appear to affect cognitive functioning, and the more demanding the cognitive task, the more task performance is affected.

Easterbrook (1959) provided an explanation for the interaction between anxiety level and task performance in attentional terms. His cue utilization theory implies that in states of high emotional arousal, for example anxiety states, subjects can use only a reduced range of cues for task performance. This limited cue utilization, which can be regarded as a narrowing of attention, puts anxious subjects at a disadvantage when carrying out difficult tasks because, compared with nonanxious subjects, they can focus on fewer of the many rellevant cues. As stated by Eysenck and Mathews $(1987 \mathrm{~b})$, this theory is inadequate because the assumption that high anxiety leads to greater concentration on some of the task cues, implies that anxiety reduces distractibility, and neither from a clinical nor from an experimental point of view (e.g. Wachtel, 1968) is this deduction very plausible. Wachtel (1967) compared attention to a beam of light; perhaps anxiety can narrow the width of the attentional beam, but that does not automatically imply that the focus of the attentional beam does not move. According to Wachtel, it is more likely that the beam shows a very distractable pattern and moves through the perceptual field. There is indeed some evidence that anxious subjects are generally more distractable if they are not certain about the location they have to attend to, and thus scan their perceptual environment (Mathews, May, Mogg, \& Eysenck, 1990). Thus, if anxious subjects have to search for a specific stimulus, they are more easily distracted by other stimuli in the environment. Mathews et al. (1990) did not find this kind of effect if the location of the stimulus had been indicated shortly before.

Eysenck (1982, 1984) argued that anxiety may not only affect performance effectiveness; but also processing efficiency. His theory concurs with the assumption that working memory is characterized by a limited capacity of central processing, and presupposes that the self-relevant concerns of anxious subjects require some portion of this limited capacity, thereby reducing the capacity available for the processing of task-relevant information. A consequence of this alternative point of view is that the debilitated performance of anxious subjects may not be attributed exclusively to a greater distractibility from the cognitive task, but also to a quantitative lack of cognitive resources available for that task. In line with this hypothesis, Ingram, Kendall, Smith, Donnell and Ronan (1987) did indeed find that anxious subjects reported more task-irrelevant thoughts than did both depressive subjects and normal controls. Although there are some indications that poor task performance is in fact associated with worry rather than with emotionality (Morris, Davies, \& Hutching, 1981), experimental evidence for the workung memory capacity theory is scarse.

To sum up, anxious subjects show impaired performance on cognitive tasks, especially on more difficult or demanding tasks. Two theories are proposed, the working memory capacity theory and the cue utilization theory, and there is little evidence to support either theory. Taking both theories together, it may be proposed that the scant cognitive capacities that anxious subjects have available for task performance are concentrated in a narrow beam of attention. When this beam 
is focused on the cognitive task, anxious subjects can perform at least as well as normal subjects. However, because of anxiety, the attentional beam may tend to "wander" across the perceptual field, severely impairing the performance of anxious subjects on the cognitive task.

\subsection{Measurement of selective attention: Paradigms}

As stated in section 2.4 it is hypothesized that anxious subjects, whille scanning the environment, will selectively allocate attention to potentially threatening stimuli. In the previous section it was argued that anxious subjects generally seem to have fewer attentional resources at their disposal, implying that anxious subjects may allocate a relatively large proportion of their attentional resources to potential danger.. Even the possibility that anxious subjects show poor attentional processing of stimuli of all possible emotional valences - except for stimuli with threatening valence - is thus consistent with this hypothesis. In section 2.4 it was explained that selective attention is measured by determining the extra attentional deployment preempted by distracting threatening stimuli, as compared to nonthreatening distractors, from a primary cognitive task. Several experimental paradigms are used to measure selective attention.

In a dichotic listening task, subjects are simultaneously presented with two different messages, one in each ear. Subjects are instructed to shadow (i.e. repeat aloud) the message presented in one ear and to ignore the message presented in the other ear. Critical words are randomly inserted into both the attended and the unattended channels. In most dichotic listening experiments subjects are asked to detect these critical words and to press an event marker whenever they hear one (Burgess, Jones, Robertson, Radicliffe, \& Emerson, 1981; Foa \& McNally, 1986; Schotte, McNally, \& Turner, 1990). Typically, subjects easily detect the critical words in the attended channel, but have great difficulty in detecting those in the unattended channel. If anxious subjects selectively allocated attention to threatening information, one would expect that anxious subjects would have relatively lowered perceptual thresholds for critical threatening words. Anxious subjects, in contrast to normal controlls, are thus expected to detect more threatening cues than riormal cues in the unattended channel. Results of several experiments confirm these expectations in phobic patients (Burgess et al. 1981) and patients suffering from bulimia nervosa (Schotte et al. 1990). Foa and McNally (1986) did not add a non. anxious control group, but in concurrence with the other experiments, they found that before treatment obsessive compulsive patients detected more obsessive com pulsive-relevant words than neutral words in the unattended channel. Cue detection can also be measured in a nonbehavioural way, by measuring psychophysiological reactions. Skin Conductance Responses (SCRs) in particular are often used for this purpose (Foa \& McNally, 1986; Schotte et al. 1.990) and, for the most part. pro. vide results comparable to the behavioural data.

Mathews and MacLeod (1986) incorporated a secondary reaction time task in their dichotic listening task, because they wanted to measure subtle attentional shifts to threatening stimuli that subjects may be unaware of. Subjects, instructed to shadow one of the messages and to carry out a reaction time task simultaneousIV. were exposed to threat or nonthreat words in the unattended channel. Both shadowing errors and probe reaction times were used as indicators of the amount of 
attentional resources the stimuli in the unattended channel required. They found that patients with generalized anxiety disorder responded significantly slower to the secondary reaction task when the words were threatening rather than nonthreatening in content. In nonanxious subjects, such a differential effect did not occur. Likewise, there was a tendency for anxious subjects to make relatively more shadowing errors as result of the presentation of threatening words. Since subjects did not remember the stimulus words they had heard, Mathews and Macleod (1986) concluded that subjects were unaware of their selective allocation of attentional resources. But Trandel and McNally (1987) doubted the unawareness of the Mathews and Macleod (1986) subjects. They argued that these subjects could have momentarily shifted attention from the attended to the unattended channel without making shadowing errors, because the prose presented through the attended channel would have included moments of silence between words and sentences. Besides, because prose is meaningful, subjects could have shifted attention for a brief moment without losing the thread of the story. Furthermore, although subjects said they did not remember the stimulus words, it was possible that they had been temporarily aware of their meaning. Trandel and McNally (1987) therefore carried out a dichotic listening experiment in which subjects were instructed to shadow a series of unrelated words and, in contrast to the Mathews and MacLeod (1986) study, they found no evidence for attentional bias in patients with post-traumatic stress disorder. It therefore remains unclear from the dichotic listening experiments whether attentional bias occurs if subjects are unaware of the stimuli presented (see also the criticisms of stimulus (un) awareness made by Holender, 1986).

Macleod, Mathews and Tata (1986) developed a rather ingenious dot probe detection task. In their task word pairs are briefly $(500 \mathrm{~ms})$ presented on a computer screen and subjects are instructed to read aloud the upper word. The moment the wörd pair disappears firom the screen, one of the words is sometimes followed by a small dot. Whenever subjects see such a dot, they have to press a button as quickly as possible. By examining the impact of threatening and nonthreatening words on the probe detection latencies in the two spatial areas, it can be determined whether visual attention had shifted towards or away from specific stimuli. Following the same line of reasoning as with the dichotic listening task it may be expected that in anxious subjects threatening words require a relatively large amount of attentional resources, resulting in a relatively rapid detection of the dot replacing such a threat cue. Macleod et al. (1986) did indeed find that anxious subjects detected the dots following the threatening words more quickly than those following the neutral words, whereas the opposite appears to be the case for normal subjects. Thus anxious subjects tend to shift attention towards threatening information, whereas normal controls tend to move attention away from such material. Comparable results are found in high- and low-trait anxious students (MacLeod \& Mathews, 1988) and patients with generalized anxiety disorder (Mogg, Mathews, \& Eysenck, 1992). Beck, Stanley, Averill, Baldwin and Deagle (1992) carried out a dot probe detection task with panic patients, and their results were roughly comparable to the MacLeod et a/. (1986) findings. Broadbent and Broadbent (1988) mentioned a possible confounder in the MacLeod et al. (1986) study and its replications, mamely that threatening words, but not controll words, were always followed by a dot probe. The appearance of a threatening word could therefore warn a subject that a dot probe would follow. In a new dot probe detection experiment, Broadbent and Broadbent (1988) ensured that both the threatening and the 
control words had an equal probability of being followed by a dot. They found that high trait anxious subjects selectively attended to threatening words, just as the anxious subjects had done in former dot probe detection tasks.

The task most frequently used in attentional bias research is the modified Stroop task. The original task (Stroop, 1935) included several cards with coloured patches or colour words. Subjects had to name the colours of the patches and to read the colour words instantly. Then, on the critical card, colour words were presented in conflicting colours and subjects had to name the colours in which the words were printed as quickly as possible, thereby ignoring the meanings of these words. The better subjects were able to ignore the conflicting word meanings, the quicker the words would be colour named. Modified Stroop tasks are used in anxiety research. In these Stroop tasks, the conflicting colour words are replaced by words with threatening and nonthreatening meanings. For example: panic patients may be presented with threatening words like "dead" and "suffocate" and nonthreatening words like "house" and "book". The task of the subjects is to name as quickly as possible the colours in which the words are printed (e.g. "red", and "blue"), and to ignore the meanings of the words. The modified Stroop tasks also take account of the principle of concurrent information: if anxious subjects selectively attend to threatening stimuli, their colour naming latencies will be longer for threatening words than for nonthreatening words. It is therefore expected that anxious subjects will be relatively slower than nonanxious subjects in colour naming threatening words. Because anxious subjects are generally slower in colour naming than nonanxious controls, a selective interference index is usually calculated by subtracting the colour naming latencies of nonthreatening words from the latencies of threatening words. In recent years computerized Stroop tasks have guaranteed more exact time measurements, enhancing the accuracy of the experiment. Another advantage of computerized Stroop tasks is that they allow for single word presentation. Single word presentation prevents subjects from reading words in advance and, by alternating the presentation of threatening and nonthreatening stimuli, stops them pondering on the threatening words. Thus, by presenting single coloured words, the influence of several potential confounders is lessened.

There has been an enormous amount of research conducted using modified Stroop tasks and because we too used this paradigm in our own experiments, a review of the literature relating to the modified Stroop task is given in a separate section (section 3.3 ).

\subsection{Specificity of the Stroop effect: Populations}

Tables 3.1, 3.2 and 3.3 give an overview of the modified Stroop experiments that have been published over the last decade. Each table summarizes the relevant characteristics and results of modified Stroop experiments. In table 3.1 experiments are presented with currently anxious subjects, and in table 3.2, comparable experiments with subjects in anxiety-related mood states. Table 3.3 presents a collection of studies in which the modified Stroop task was applied to subjects in other mood states, such as depression, and to subjects with certain coping styles, like repression. 


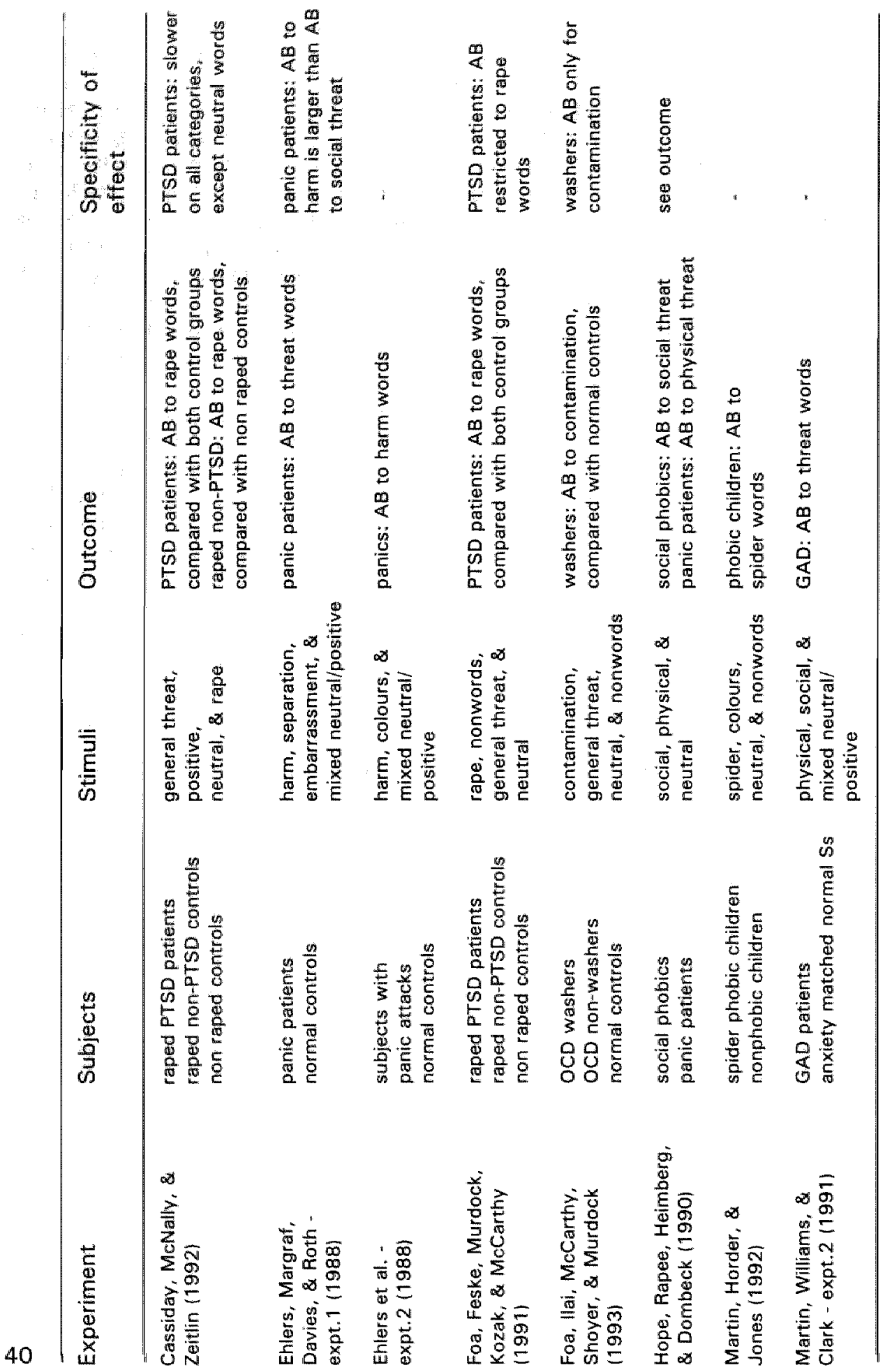




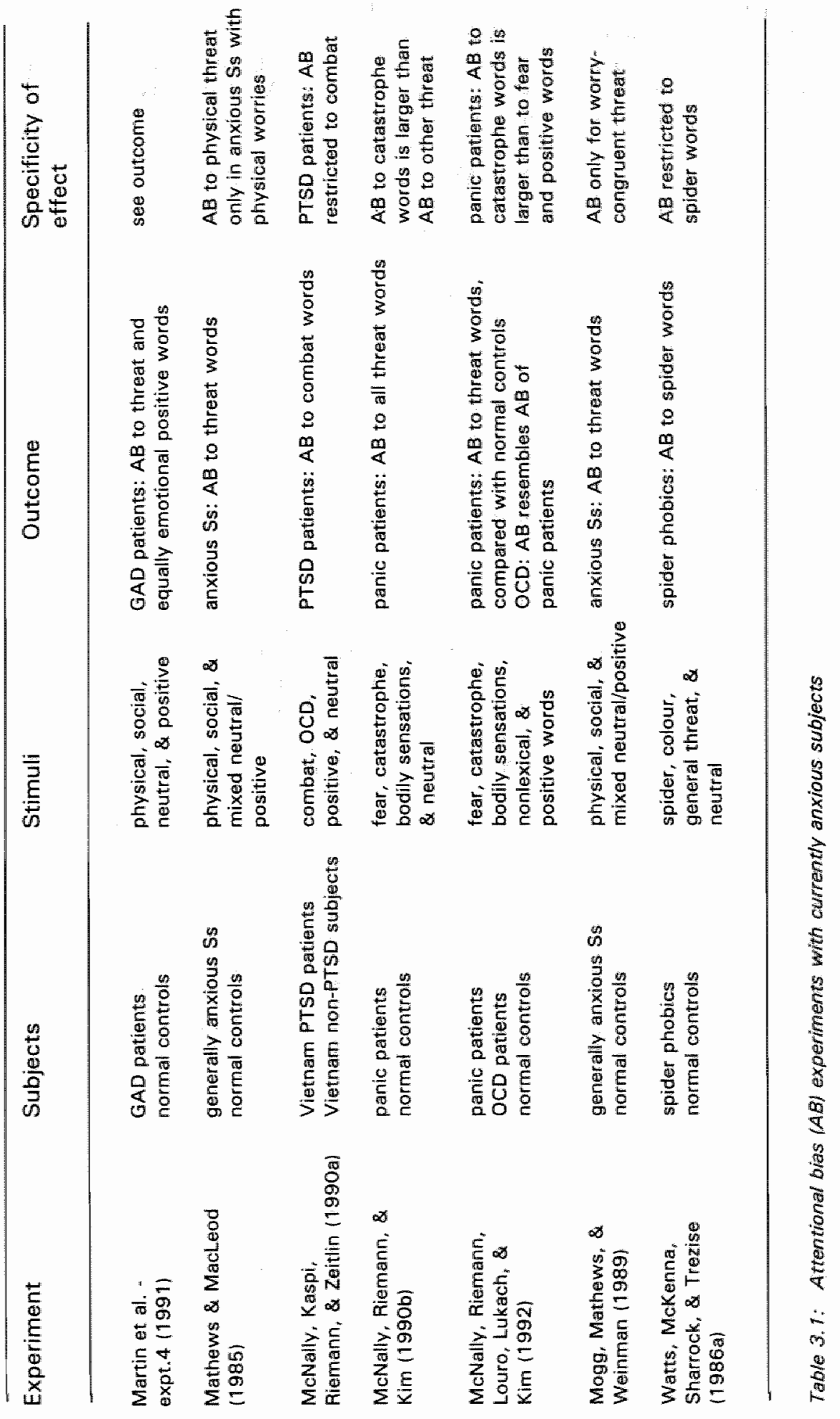


As can be seen in table 3.1, in the last decade all DSM-1II-R anxiety disorders have been subjected to Stroop examination. Word presentation and computation of time differed largely. In some experiments words were written on a card and time was measured using a stopwatch le.g. Ehlers, Margraf, Davies, \& Roth, 1988; Mathews MacLeod, 1985; McNally, Kaspi, Riemann, \& Zeitlin, 1990a; Watts, Mckenna, Sharrock, \& Trezise, 1986a). In other experiments each word was presented separately on a computer screen and the time was measured using an advanced computer clock le.g. Cassidy, McNally, \& Zeitlin, 1992; Foa, Feske, Murdock, Kozak, \& McCarthy, 1991; McNally, Riemann, \& Kim, 1990b). The results of these various experiments are however remarkably consistent: clinically anxious subjects are relatively slow in colour naming words that, to them, have a threatening meaning. These consistent results are the more astonishing, when one considers the magnitudes of the attentional bias effects: the deterioration in colour naming threatening words usually does not exceed some dozens of milliseconds per stimulus. The colour naming latencies of control stimuli having been taken into account, anxious subjects attend to threatening stimuli only some milliseconds longer than do nonanxious subjects.

It is not only anxious adults who appear to attend selectively to threatening stimuli. Martin, Horder and Jones (1992) examined spider phobic children and found attentional biases towards spider-related stimuli in children as young as six or seven years old. Yet more interesting was their finding that the magnitude of the observed attentional biases in these very young children did not differ significantly from those of children aged twelve or thirteen. Comparing their data to those of the Watts et al. (1986a) experiment, Martin et al. (1992) reported that the attentional biases of the children were even remarkably close to the attentional biases of adiult spider phobics. There is thus reason to believe that as long as the subjects are anxious, the attentional biases may be small, but stable phenomena.

However, attentional bias effects do not seem to be unalterable. When spider phobic subjects participated in a behavioural treatment programme in order to reduce their phobic anxiety, their attentional biases were also significantly reduced (Watts et al., 1986a). The Stroop paradigm, then, provides evidence that clinically anxious subjects show an attentional bias in favour of fear-relevant information. This attentional bias seems to be relatively stable, but not unalterable.

Selective attention is not unique to clinically anxious subjects. As is shown in table 3.2, several studies have demonstrated attentional bias effects in subjects in anxiety-related mood states. Eating disorder subjects in particular are frequently investigated. Both subjects with anorexia nervosa and subjects with bulimia nervosa attended selectively to words related to food and eating (Ben-Tovim \& Walker, 1990; Ben-Tovim, Walker, Fok, \& Yap, 1989; Channon, Hemsley, \& de Silva, 1988; Cooper, Anastasiades, \& Fairburn, 1992; Fairburn, Cooper, Cooper, McKenna, \& Anastasiades, 1991). Whether eating disorder subjects also show an attentional bias towards words related to body shape (Ben-Tavim \& Walker, 1990) or not (Ben-Tovim et al., 1989) remains unclear and may depend on the subjects under review.

In several of the experiments presented in table 3.2, modified Stroop tasks were given to normal subjects in an anxious mood state. Giles and Cairns (1989) for instance presented a modified Stroop test to native Northern Irish subjects, English subjects presently living in Northern Ireland and English subjects living in England. 


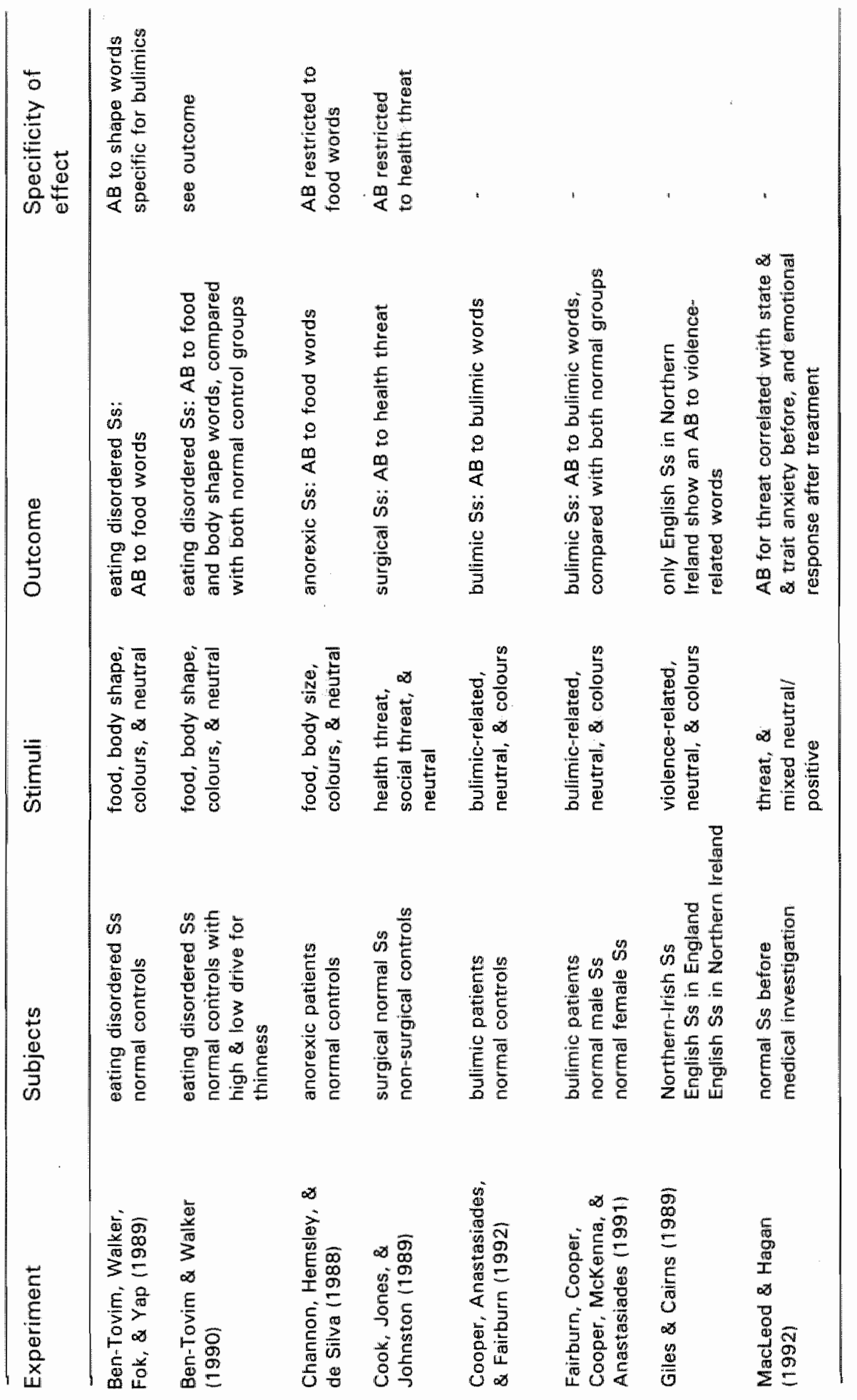




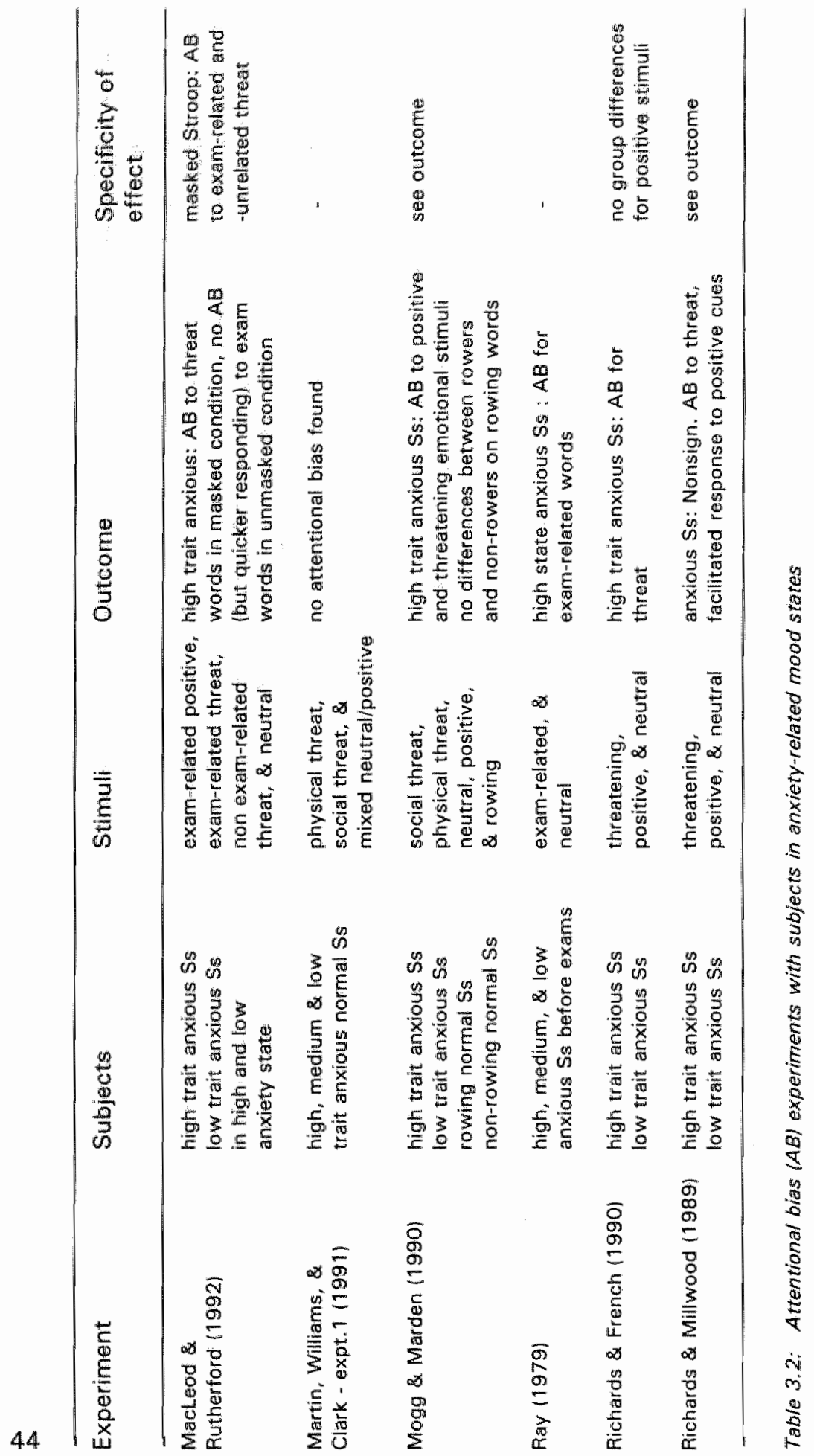


Only the English subjects living in Northern Ireland showed an attentional bias to violence-related words. Cook, Jones and Johnston (1989) presented Stroop cards to subjects facing a minor surgical operation and to non-surgicall control subjects. The surgical subjects were slower in colour naming the health-threath related words than in colour naming the neutral control words. MacLeod and Hagan (1992) tested subjects awaiting medical investigation. They presented coloured words under masked and unmasked exposure conditions and found that selective attention to threatening stimuli, demonstrated under masked exposure conditions, correlated positively with trait and state anxiety before medical treatment. Moreover, this selective attention also correlated to later emotional reaction to subsequent diagnosis of pathology. In view of the above-mentioned studies, it may be suggested that normal subjects that are actually anxious may show similar attentional biases to threatening information as do anxiety patients.

The Stroop experiments discussed thus far provide evidence that attentional bias is associated with current anxiety. Several experiments presented in table $3.3 \mathrm{claim}$ that attentional biases also occur in mood states unrelated to anxiety (e.g. Bentall \& Thompson, 1990; Gotlib \& McCann, 1984; Pearce \& Morley, 1989; Williams \& Broadbent, 1986). In some of these experiments it can actually be questioned whether anxiety is really of no influence. Considering the co-morbidity between anxiety and depression. Williams et al. (1988) for instance doubted whether the depressive subjects in the Gotlib and McCann lexperiment 1. 1984) study were not anxiouis. And Williams and Broadbent (1986) reported that the suicide attempters were not only more depressed, but also more anxious than the control subjects. in other studies, however, anxiety does not seem to be of considerable influence. In two studies normal, undisturbed subjects were differentiated on the basis of their scores on a personality scale. Bentall and Thompson (1990) compared subjects with high, medium and low scores on the Eckbllad \& Chapman's Hypomanic Personality Scale and found that hypomanic traits were associated with retarded colour naming of depressive, but not manic, words. Dawkins and Furnham (1989) differentiated subjects with high and low anxiety scores and subjects with a repressive coping style, i.e. subjects scoring low on anxiety scales, who are shown to be anxious but reluctant to admit it. They found that repressive subjects showed larger attentional biases for threatening words than highly anxious subjects. Attempts to repress a certain mood state, either anxiety or depression, thus may result in an attentional bias for stimulus words relevant to that mood state.

It would seem, then, anxiety disordered subjects may be characterized by selective attention to threatening information, but that this cognitive bias is certainly not restricted to anxiety disorders. The variety of populations in which an attentional bias is shown would, in fact, seem to indicate that people selectively attend to all stimuli associated with their current concerns and interests. Although the results of some experiments may be interpreted as confirming this statement /Channon \& Hayward, 1990; McKenna, 1986), there are some compelling contradictory findings. Mogg and Marden (1990) tested students who were, or were not, members of a rowing club and found that rowers did not selectively attend to rowing words. A more direct test was provided by Wegner and Erber (1992). They instructed some of their subjects to concentrate on a certain topic and these subjects were not slower in colour naming stimuli related to this topic than in colour naming other stimuli. The conditions under which attentional bias may occur require further investigation. Some topics of possible interest will be discussed in the next sections. 


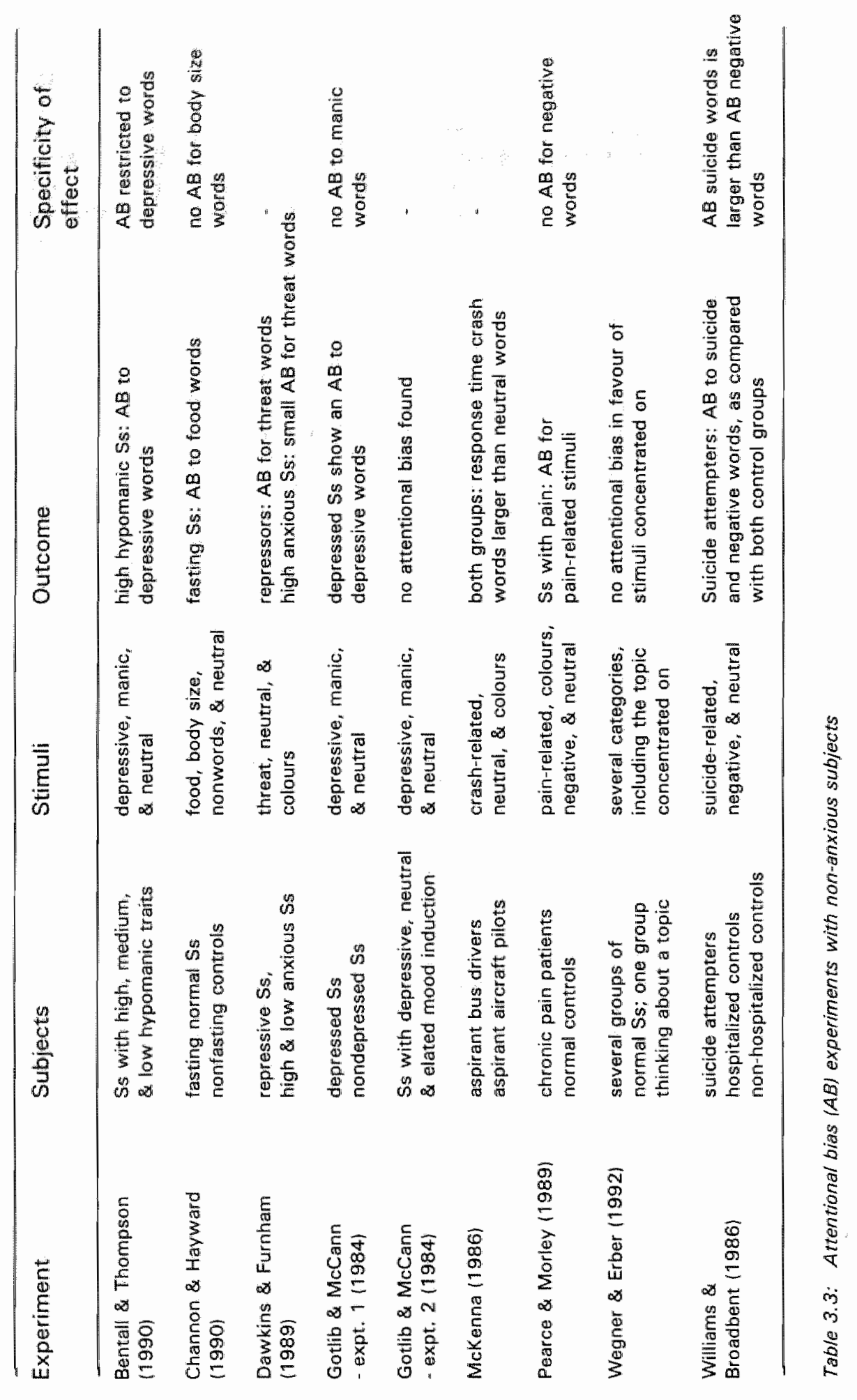




\subsection{The state-trait problem: Are current mood or enduring factors causal to attentional bias?}

One of the most widely discussed topics is whether attentional bias is merely associated with state anxiety or with trait anxiety. Reformulated, the question is whether anyone in an anxious mood would selectively attend to potential danger or whether the tendency to show such bias is restricted to a specific subgroup of subjects.

Originally Macteod et al. (1986) argued that the occurrence and nonoccurrence of attentional bias would be determined by a certain threshold level of trait anxiety, whereas state anxiety would determine the intensity of that bias. In more recent studies, the relationship between state anxiety, trait anxiety and attentional bias was been less precisely formulated. For instance, MacLeod (1990, p.18) suggested that attentional bias may be "....mediated by neither state nor trait anxiety alone, but rather by the interactive influence of both these variables" "

Correlational data does little to clarify the differential associations between trait and state anxiety and attentional bias. Mathews and MacLeod (1985) found that state anxiety better than trait anxiety predicted the delay in colour naming threatening words, but in a replication study by Mogg et al. (1989) exactly the opposite effects were found. MacLeod and Hagan (1992) tested normal subjects and found, under masked exposure conditions, that state and trait anxiety correlated equally and significantly to the delay in colour naming threatening words. It is not surprising that it is difficult to find differential evidence for state and trait anxiety in the allocation of attention in these correlational studies, since trait and state anxiety themselves tend to show extremely high correlations (MacLeod, 1990; Merckelbach, van Hout, van den Hout, \& Lavy, 1990ay.

As can be seen in table 3.2, high and low trait anxious subjects were compared in several modified Stroop experiments. Mogg and Marden (1990), Richards and Millwood (1988), and Richards and French (1990) reported that high trait anxious subjects selectively attended to threatening stimuli. However, both in the Richards and Millwood (1988) and the Richards and French (1990) study the high trait anxious group was also significantly more state anxious than the low trait anxious group. And Mogg and Marden (1990) failed to report the state anxiety scores of the trait anxiety groups. In contrast with the previous three studies, Martin, Williams and Clark (experiment 1, 1991) did not find evidence for attentional bias in high trait anxious normal subjects. MacLead and Rutherford (1992) attempted to dissociate trait and state anxiety by dividing normal students into low and high trait anxiety groups, and by testing them on two occasions: before and after an important examination. They found different effects for masked and unmasked exposure trials. Under unmasked exposure conditions there was a (marginally significant) interaction between the time of testing and meaning of the stimulus words presented. That is, prior to the examination both the high and the low trait anxious subjects tended to attend selectively towards threatening words. Under masked exposure conditions, there was a significant interaction between trait anxiety, time of testing and stimulus valence. MacLeod and Rutherford $(1992$, p.487) concluded that under masked exposure conditions "... the pattern of selective processing does indeed appear to have been mediated by the precise interactive function of trait and state variables...". However, on both test occasions the high and low trait anxiety group differed not only in trait anxiety, but also in state anxiety level. Since the 
level of state anxiety was not taken into account in the statistical testing, the MacLeod and Rutherford's (1992) conclusion may be somewhat premature.

It is questionable, not only on clinical, but also on conceptual grounds, whether high trait anxiety, independent of state anxiety, may affect attentional processing. The general distinction between states and traits has been the subject of conceptual discussions (Allen \& Potkay, 1981; Fridhandler, 1986). It is persuasively argued that personality traits like trait anxiety will not be continuously manifest, but rather will reflect a stable proneness to respond to stressful situations with a state of anxiety (Fridhandler, 1986). According to this view, trait anxiety is an abstract entity in itself, not to be directly observed or measured, finding expression in concrete periods of state anxiety. The assessment of trait anxiety, independent af state anxiety, will therefore be a complex, if not impossible, task. There are indeed indications that measurements of trait anxiety are affected by current state anxiety and other transient influences (e.g. Smorenburg, van der Ent, \& Bonke, 1986; Steyer, Majcen, Schwenckmezger, \& Buchner, 1989). If the assessment of trait anxiety itself is affected by various situational influences, it may be obvious that the stateindependent influence of trait anxiety on the allocation of attention can barely be established.

The fact that transient factors, like state anxiety, are hard to exclude is not the only argument against the hypothesized role of enduring personality traits. Several empirical findings suggest that attentional bias is quite a flexible phenomenon. Firstly, there is some evidence that attentional bias can be induced in normal subjects (Channon \& Hayward, 1990; Merckelbach, van Hout, de Jong, \& van den Hout, $1990 \mathrm{~b})$. And secondly, attentionall bias effects in anxious subjects are seriously affected by a decrease in current anxiety; both Watts et al. (1986a) and Foa and McNally (1986) found that the attentional bias of anxious subjects was elliminated after treatment.

The question remains, then, as to the relevance of the state-trait problem for the cognitive model of attentional bias. It should be noted that the concepts of trait anxiety and cognitive structures are not directly comparable, because they are not of the same level. An enduring personality state can be described in terms of personality traits ${ }_{r}$ and explained in terms of cognitive structures. It would be beyond the scope of the current section to discuss whether these concepts can be linked. Certainly there are some similarities; an important one is that both trait anxiety and cognitive structures, like danger schemata and fear networks, refer to an enduring and relatively stable phenomenon in a specific subgroup of subjects. If attentional bias indeed reflects the activity of enduring cognitive fear structures, its form should not be heavily dependent on transient factors. The reactivity of attentional bias, i.e. the swiftness to which attentional bias adapts to a subject's (learning) experiences, thus becomes interesting topic. Strong reactivity of attentional bias would not only be at odds with the trait anxiety hypothesis, but also with the cognitive model of attentional bias.

\subsection{The familiarity problem}

It is well known that words used frequently have more attention catching power than have rare words (e.g. Klein, 1964), and consequently produce greater interference in colour naming. It is therefore suggested (Foa \& McNally, 1986) that anxious 
subjects may take longer to colour name threatening words, not because of the meaning of the words, but because these words are more familiar to them. Several experiments matched threatening and control words in terms of frequency of usage (e.g. Mathews \& MacLeod, 1985; McNally et al, 1990a; Mogg et al., 1989). Objective frequency norms, however, do no take into account the notable individual differences in the frequency of usage. It is likely that, for anxious subjects, words specifically related to their fears are more common than is rendered in frequency tables. These tables can therefore not be used to rule out the possibility that familiarity is causal for attentional bias in anxious subjects.

Two treatment effect studies (Foa \& McNally, 1986; Watts et al., 1986a) provide convincing arguments against the familiarity hypothesis. As a result of behavioural treatment, including an exposure programme, the famillarity with threatening stimuli was certainly not reduced, even probably increased. However, the attentional bias effects were significantly reduced. McNally et al. (1990b) tested panic patients and used clinicians, experienced in treating these patients, as control subjects. More or less on face value they argued that these clinicians were as familiar with panic-related stimuli as were panic patients. MoNally et al. (1990b) found that despite the supposed comparable familiarity " panic disorder patients selectively attended to threatening stimuli. Taking together, these experiments offer no reason to believe that familiarity is of vital interest in explaining the attentional bias effects in anxious subjects.

\subsection{Specificity of Stroop effect: Stimuli}

The specificity of the Stroop interference was been investigated in several attentional bias studies. In studies with generalized anxiety patients (Mathews \& MacLeod, 1985; Mogg et al., 1989) and panic patients (Ehlers et al., 1988), subjects were divided on the basis of their primary concern: physical or social wellbeing. Social and physical worriers showed different patterns in colour naming latencies (Mathews \& MacLeod, 1985; Mogg et al., 1989), but in at least one study no differences between the two subtypes could be found (Ehlers et al., 1988). Hope, Rapee, Heimberg and Dombeck (1990) tested panic patients and social phobics in one experimental study and found that they had attentional biases specific to physical and social threat words respectively.

There are thus some indications that the attentional bias that anxious subjects show is more pronounced for worry-congruent than for worry-incongruent materiall. But it is also suggested that anxious or otherwise emotionally disturbed subjects may attend selectively to general emotional stimuli. In several experiments, subjects were therefore not only presented with specific threat and neutral control words but also with general, either positively or negatively valenced, emotional words. Mixed results were obtained from these experiments. It is evidenced that in anxious/emotionally disturbed subjects the presentation of general emotional words may slow down collour naming (e.g. Cassidy et al., 1992; Mogg and Marden, 1990; Williams and Broadbent, 1988). But in other experiments no significant differences in colour naming general emotional words were found (e.g. Foa et al., 1991; McNally et al., 1990a: Watts et al., 1986ai).

A possible solution to these conflicting results came from Martin, Williams and Clark (1991). Martin et al. (experiment 3,1991) pointed out that the control words 
used in attentional bias studies seem to be less emotional than the specific threat words. In order to test this idea, they analyzed the emotionality ratings of threatening and control words of six attentional bias studies, and indeed found that in all these studies the threatening words were more emotional than the control words. In a subsequent attentional bias experiment Martin et al, (experiment 4,1991 ) ensured that the specific threat words presented were as emotional as the positively valenced control words. Results of this study confirmed that anxious subjects not only show an attentional bias for threat words, but also for positively valenced emotional words. Consequently Martin et al. (1991) stated that anxious subjects do not selectively attend to threatening stimuli, but to emotional stimuli in general.

A general attentional bias to emotional words would not concur with the premisses of cognitive theories. Stimulus specificity, and especially Martin et al.'s (1991) emotionality hypothesis, must in future research therefore be submitted to critical examination.

\subsection{Memory bias}

As is described in section 2.6, anxious subjects often show an attentional bias, but no subsequent memory bias. The explanation most widely used is that anxious subjects, after detection and identification of the threatening stimuli, use avoidance strategies and do not elaborate the threatening information in further detail (see further section 2.6). Some psychophysiological findings in anxiety patients are in accord with this argumentation. It is demonstrated that anxiety patients, confronted with fear-relevant stimuli, may show biphasic heart rate response patterns: an initial deceleration is sometimes followed by an acceleration. Some authors have conceptualized these phases in the response patterns as indices of the orienting and defensive reaction respectively (for relevant overviews see Hughdahl, 1989; Sartory, 1983).

Results from several cognitive experiments provide more direct evidence for the assumption that anxious subjects do not elaborate threatening information very well. For instance Watts, Sharrock and Trezise (1986b) asked spider phobic and nonanxious controls to imagine spiders, and reported that these images were less detailed in spider phobics than in control subjects. And Borkovec and Sides (1979) found that the vividness of fear-relevant imagery in phobic subjects increased as a result of a desensitization procedure. Consistently, the results of an experiment by Landau (1980) suggested that dog phobics have less detailed knowledge about dogs than do nonphobic subjects.

Watts and Coyle (1992) based a memory bias experiment on the a priori assumption that the anxiety of animal phobics is merely directed to stimulus characteristics, and not to anxiety responses. If avoidance is the cause of the lack of elaboration and subsequent memory bias effects, they argued, in spider phobics memory bias may be demonstrated for threatening response words (such as "scared" and "shock") but not for threatening stimulus words (such as "cobweb" and "legs"). Data failed to support this hypothesis, but this failure may be due to a faulty a priori assumption. Arntz et al. (1993b) demonstrated that spider phobics are not only afraid of spider-related stimulus words, but also of their own anxiety responses. The possibility remains open, therefore, that anxious subjects who selectively attend to threatening stimuli, subsequently avoid elaboration and storage of these stimuli. 
As is stated in the previous section there is some evidence that anxious subjects not only show an attentional bias towards threatening wolds but also towards other, nonthreatening emotional words. Avoidance strategies would be of no use in the case of positively valenced emotional words. Consequently it can be suggested that an attentional bias towards positively evaluated stimuli would be associated with selectively enhanced memory of these stimuli. If such an association could be demonstrated, this would lend additional support to the suggestion that the failures in finding selective memory effects in anxious subjects who do selectively attend to threatening stimuli, must be attributed to avoidance strategies.

\subsection{The significance of attentional bias}

Ever since attentional bias is one of the focal points of cognitive experimental research, people have been wondered what its significance could be. The most nihilistic suggestion made, is that attentional bias has no etiological significance, but simply is an epiphenomenon of anxiety. For instance, MacLeod et al. (1986) gave an alternative explanation for anxious subjects showing selective delay in colour naming threatening words. They suggested that this delay may not be the result of the allocation of attentional resources, but of emotional arousal resulting from the presentation of threatening stimuli. In other words, they suggested that a modified Stroop task measured impaired response, not selective attention (for a general critical discussion of Stroop interference, see MacLeod, 1991). For a number of reasons this suggestion does not provide a satisfactory explanation for attentional bias effects. Firstly, if attentional bias is caused by arousal, it is unclear as to why arousal results in impaired performance on the Stroop task, and in facilitated performance on the dichotic listening task and the dot probe detection task. Secondly, in a computerized Stroop task, differently valenced words are intermingled and it is difficult to imagine how arousal can be switched on and off with each single word presented. Thirdly, it is known from several experiments (see tables 3.2 and 3.3 ) that attentional bias does not only accur in subjects with strong anxiety responses, but also in subjects with rather mill anxiety or no appreciable anxiety at all.

Several authors le.g. Eysenck \& Mathews, 1987b; Hope et al., 1990) more or less explicitly suggested that selective allocation of attentional resources may predispose an individual to anxiety disorders. Merckelbach et al. (1990a) pointed out that these authors interpret the mutual relationships between anxiety and attention as follows: high trait anxiety, which is mostly genetically determined "leads to a tendency to orient towards the location of potential threat (attentional bias). High state anxiety, or even an anxiety disorder, may then, in long term, result from this attentional bias. Although a very popular point of view, there is little evidence for this notion. Firstly, the role of trait anxiety can be questioned. As argued in section 3.4 , it is difficult to demonstrate an attentional bias for threatening information in subjects, who are merely high trait anxious. Secondly, although it sounds very plausible, up to now no experimental data has provided evidence that attentional bias results in heightened state anxiety.

Foa and Kozak (1992) offered an alternative explanation, closely linked to both the concept of familiarity and Lang's (1977, 1979, 1985) theory of fear structures in anxiety disorders. They pointed out that more meaningful words cause more interference in collour naming. According to Foa and Kozalk (1992), meaning may be 
reflected in the number and pattern of associations representing that word. A more meaningful word would trigger a more elaborate memory structure, consequently using more processing capacity and leaving less capacity for the primary task of colour naming than a less meaningful concept would do. They referred to a study of Hasher, in which it was found that more colour naming interference is shown for words, known in a certain context, than for isolated words. They adopted Lang's theory and suggested that anxious subjects may have relatively complex fear structures, in which propositions about stimulus, response and meaning are strongly associated and therewith readily accessed. In other words, they hypothesized that in anxious subjects the fear structures are extended and elaborated. If one of the many associated propositions is triggered, activation is spread throughout the complete structure and little processing resources are left for the colour naming task.

Several authors have claimed that attentional bias may have a behavioural function. They suggested that selective attention towards potential threat prepares subjects optimally to avoid or escape potentially threatening situations (for more or less explicit references to this hypothesis, see e.g. Litz \& Keane, 1989; MacLeod et al. , 1986; MacLeod \& Mathews, 1991; Mathews, 1989; Mogg et al., 1987). From this point of view selective attention to threatening and threat-related information may be adaptive for an organism. Because increased attention can enhance early detection and identification of potential danger, avoidance and escape responses may be facilitated.

It should be noted, however, that, although the potential adaptiveness of attentional bias is regularly brought to the fore, this hypothesis has never been tested. It is therefore unclear whether the attentional bias of anxious subjects can be interpreted more generally as an attentional bias towards stimuli that have to be responded to quickly. Before such interpretation can be admitted, several issues have to be investigated. Firstly, the adaptiveness hypothesis implies that selective attention is not only found for threatening stimuli, but for all stimuli that require a quick response. Secondly, another implication of this hypothesis is that nonanxious subjects will also selectively attend to stimuli that require immediate action. Thirdly, whether attentional bias does indeed promote quick response requires investigation.

The adaptiveness hypothesis not only lacks empirical evidence, its theoretical foundation has also not been elaborated. The adaptiveness hypothesis may be incorporated into cognitive theories; Lang's theory about fear structures (Foa \& Kozak, 1986; Lang, 1977, 1979) in particular allows for such incorporation. According to this theory, programmes of escape and avoidance may become activated easily by selective attention to threatening stimuli, while, of course, other meaningful stimuli may activate other response programmes. Whatever response programme becomes activated, it may enable a subject to respond quickly and adequately. Since the adaptiveness hypothesis itself has not, as yet, been tested, such theoretical elaboration may be rather previous at the moment. These theoretical considerations are therefore discussed further in chapter 6.

\subsection{Ecological validity of attentional tests}

A somewhat different question is whether the impaired or enhanced performances on laboratory attention tasks reproduce faithfully selective attention, as it occurs in natural circumstances. This is merely the problem of ecological validity. 
Modified Stroop tests, dichotic listening and dot probe detection tests, all make use of linguistic stimuli. Many stimuli feared by anxiety patients are, however, perceptual objects: simple phobics may be frightened by dogs or spiders, patients with obsessive compulsive disorder may be frightened by dirty door-handles, and social phobics by angry faces. Other anxiety patients fear specific situations, like being trapped in an elevator, or travelling by train.

of course the linguistic stimuli used in the attentional bias experiments can refer to these feared objects or situations. But it is questionable whether this linguistic approach is always preferable or whether, alternatively, pictorial stimuli may sometimes offer more valid representations of the feared stimuli. It is puzzling why pictorial stimuli are so rarely used in attentional bias research. As far as the author knows pictorial stimuli have, as yet, only been used in a few studies. Von Streblow, Hoffmann and Kasielke (1985), testing patients with various phobic complaints, used a creative pictorial paradigm. Subjects were exposed to a series of slides, made up of three diagonally presented pictures; their instruction was to categorize the central picture and to ignore the flanking ones. The flanking pictures were meant to distract subjects from categarizing the central pictures, just as in a modified Stroop task the meaning of the words is meant to distract the subject from colour naming. Despite the promising paradigm, the study suffered from so many methodological flaws, that interpretation of the results was problematic.

Achille, Stewart, Dubois-Nguyen, Peterson and Pihl (1991) tested socially anxious and nonanxious female subjects with a pictorial attentional bias task. The subjects were presented with slides of ambiguous facial expression and were instructed to react as quickly as possible to the shape of a target object placed in the centre of each face. Achille et al. (1991) found that socially anxious subjects responded more quickly: to the target object when the expression of the face surrounding the object contained threatening components. Achille et al. 11991$)$ thought this finding supported the attentional bias hypothesis, but in fact their results can only led to the conclusion that anxious subjects are better able than normals to ignore threatening information! Thus the findings of Achille et al. (1991) curiously represent one of the few failures to demonstrate attentional bias effects in anxious subjects.

A third pictorial test by Merckelbach et al. (1990b) demonstrated that attentional bias can be the result of classical conditioning. They first exposed normal, nonanxious subjects to two slides with angry faces, one of which was followed by an electric shock. In the subsequent attentional bias test, these angry faces were combined with sets of three numbers and the subjects were instructed to add up these numbers as quickly as possible and to verbalize the results. The subjects were slower in adding up the numbers combined with the reinforced slide than in adding up the numbers combined with the unireinforced slide. Merckelbach et al. (1990b) concluded that the nonanxious subjects selectively attended to the angry face that was formerly followed by an electric shock.

The studies, then, that have used pictorial stimuli are few in number and largely unsound. This is regrettable since the ecological validity may be more evident in pictorial stimuli. 


\subsection{Summary}

One of the firm conclusions to emerge from this chapter is that anxiety and anxiety-related states are associated with an attentional bias to threat-relevant information. Further, various experiments have demonstrated that nonanxious subjects may show an attentional bias for specific, meaningful stimuli. In none of these studies have subjects shown an associated memory bias. It is sometimes suggested that these failures to find selective memory effects may be due to avoidance strategies, but up to now there is no evidence to support this hypothesis.

The role of trait anxiety is rather obscure. High and low trait anxious subjects have been investigated in several experiments and it has been demonstrated that high trait anxious subjects selectively attend to threatening stimuli. However, the high trait anxious subjects were also more highly state anxious. It is unclear, therefore, whether purely high trait anxiety, independent of high state anxiety, can affect attentional processing. Cognitive theories assume that attentional bias reflects enduring and complex cognitive structures, but there are some tentative indications that attentional bias effects are heavily dependent on current mood states. The reactivity of attentional bias to new experiences needs clarification. The role of trait anxiety and other enduring (cognitive) factors may subsequently be determined.

It is suggested that anxious subjects show an attentional bias towards threatening words because they are more familiar with these words. Several experiments have dealt with this issue and it seems clear now that familiarity is not of particular importance in explaining attentionall bias.

Regarding the specificity of the attentional bias effects in anxious subjects, it has been hypothesized that anxious subjects selectively attend to emotional stimuli in general; whether related or unrelated to the anxiety problems. There is one experiment that supports this hypothesis, but more research is needed.

With regard to the potential significance of selective attention, it has been hypothesized that attentional bias may be adaptive for survival. Selective attention to specific and meaningful stimuli may facilitate a quick and adequate response to these stimuli. Although mentioned frequently, this hypothesis has never been tested and is thus still very speculative.

The ecological validity of linguistic attentional bias tests has been discussed in the previous section. Although there are good arguments against the use of linguistic tests, pictorial stimuli have been used in only a few experiments. Because of the lack of solid experiments in which anxious subjects are presented with pictorial stimuli, it is unclear whether pictorial and linguistic tests provide similar results. 


\section{Chapter 4}

\section{Introduction to the experimental studies}

The previous chapter deals with several topics of interest, each of which could serve to broaden the extent of our knowledge of the nature and potential significance of selective attention. Each of these issues has also, more or less extensively. been a subject of our investigations. In this chapter, an overview is given of the experimental studies. This overview should be considered as being a matrix of research issues and experimental studies; some studies deal with several research issues, and some research issues are addressed by several studies. For ease of survey, the present chapter centres the experiments around the various topics of interest. 


\section{Replication of earlier findings}

Our first aim was to replicate the attentional bias effects in anxious subjects. Using the well-known Stroop paradigm we demonstrated that spider phobics and absessive compulsive patients selectively attend to spider-related words (see section 5.11 and threatening obsessive compulsive-related words respectively (see section 5.2). These replicative findings formed the basis of further and more refined investigations.

\section{Reactivity of attentional bias}

In chapter 3 two treatment studies were discussed, in which the elimination of attentional bias was demonstrated (Foa \& McNally, 1986; Watts et al., 1986a). Although it is now known that the allocation of attention can be altered, the question remains as to how quickly such adaptation to new experiences can take place (see also section 3.4). Cognitive theories suppose that selective attention is a reflection of highly organized cognitive fear structures. A permanent reduction in attentional bias may then only be expected if cognitive distortions are repeatedly falsified and fear structures are readjusted to new and more realistic insights. It is likely that such readjustment of cognitive structures will need a certain amount of time, and that consequently selective attention will not be very reactive to sudden changes in anxiety levels. In both the Foa and McNally (1986) and the Watts et al. (1986a) treatment studies, subjects were treated intensively for several weeks, and such lengthy treatment programmes preclude definite conclusions about the reactivity of attentional bias effects. In three experiments, we tested spider phobics before and after a one- (see section 5.1), or two-session (see section 5.6 and section 5.7) exposure treatment. The application of such a highly circumscriptive and time-limited exposure treatment programme allowed for more direct investigation of the reactivity of selective attention. As a result of this treatment programme, anxiety is strongly reduced in 2-4 hours time, whereas drastic changes in trait anxiety or other enduring (cognitive) factors cannot be expected in such a short period of time. The one- and two-session exposure treatments seem therefore to have outstanding potential for testing the pure effects of current anxiety on attentional bias.

\section{Familiarity of the stimuli}

There are several convincing arguments against the hypothesis that anxious subjects show an attentional bias towards threatening stimuli because these stimuli are more familiar to them (see section 3.5). This issue is therefore not explicitly investigated further in the chapter 5 experiments.

\section{Stimulus specificity}

Stimulus specificity is indirectly considered in two experiments (see section $\mathbf{5 . 1}$ and section 5.3\%. In both studies, three categories of words were presented to the subjects: relevant words, neutral control words and control words with the same emotional direction as the relevant words. The emotional direction of neutral and emotional control words were checked in pilot studies, but no care was taken beforehand to check for the intensity of emotional valence. That is, like the relevant 
words, the emotional control words were negatively (see section 5.11 or positively (see section 5.3) valenced, but it was not ensured pre-experimentally that these words were as negative or as positive as the relevant words. In the section 5.3 experiment, such a manipulation check was carried out only after the Stroop task performance. Results of these experiments can therefore give only a tentative indication of whether the attentional bias is specific to relevant words or as suggested by Martin et al. $(1991 \%$, it is shown for all emotional words. Stimulus specificity was however the central topic of a study with obsessive compulsive patients (see sec. tion 5.2). Obsessive compulsive patients were presented with a Stroop task, which included five word categories: one neutral category and four emotional categories. These four emotional categories consisted of positively and negatively valenced words, either related or unrelated to the obsessive compulsive compllaints. Words from the four emotional categories were comparable on strength of emotional valence. The design of this experiment allowed for careful examination of the emotionality hypothesis.

\section{Memory bias}

As stated in section 3.7, anxious subjects who selectively attend to threatening stimuli, do not selectively remember these stimuli afterwards. This inconsistency has been attributed to a lack of elaboration; once an anxious person has detected a threatening stimulus, avoidance strategies are thought to become activated and further elaboration of threatening information will therefore be suppressed as far as possible. In section 3.7 it was argued that such avoidance response does not account for selective attention towards positively valenced stimuli. If the suggestion is correct that anxious subjects do not show a memory bias for threatening information because of avoidance responses, it can be expected that an attentional bias to positively valenced information will be associated with a subsequent memory bias for that information. In section 5.3, this hypothesis is tested.

\section{Ecological validity}

A methodological issue was raised in section 3.9: it was questioned whether the use of linguistic tests is always preferable, since the majority of anxious subjects seem to fear perceptual objects or situations. Pictorial tests may thus have greater ecological validity than linguistic tests. In two studies, presented in sections 5.6 and 5.7, pictorial tests are used to investigate the attentional bias effect in spider phobics. In the section 5.6 experiment we used a pictorial flanker paradigm, similar to the Von Streblow et al. (1985) paradigm that was described in section 3.9. In the section 5.7 experiment, both pictorial and linguistic stimuli were used. Linguistic stimuli were presented according to the well-known Stroop paradigm. The pictorial stimuli were variations of these linguistic Stroop stimuli, i.e. pictures were presented in coloured circles and subjects were instructed to name the colours as quickly as possible and to ignore the meaning of the pictures.

\section{Adaptiveness of attentional bias}

In section 3.8, the potential behavioural function of attentional bias was dis* cussed. It was speculated that an attentional bias for threatening of otherwise meaningful stimuli could be adaptive. Increased attention towards these stimuli may enables a subject to respond quickly and adequately to important stimuli. This matter is the main topic of three experimental studies. Two issues are investigated: (1) 
whether attentional bias indeed facilitates quick responding, and (2) whether nonanxious subjects show an attentional bias for nonthreatening stimuli that require a quick response. The first issue is raised in the section 5.6 experiment, which is an investigation of the hypothesized association between attentional bias and facilitated escape in spider phobics. A pictorial flanker paradigm is used, similar to that of Von Streblow et al. (1985). Such a task can be used to test for a correlational, but not a causal, relationship between attentional bias and speed of response. Thus, it could be objected that a third, independent factor may be causal to both the attentional bias and the facilitated response. The second issue, whether nonanxious subjects show an attentional bias towards urgent stimuli, is investigated in two experiments (see section 5.3, and section 5.5). These experiments with normal, nonanxious subjects allowed for randomization, thereby reducing the possible influence of confounding factors. In both experiments one group of subjects was exposed to experimental procedures, intended to alter the meaning of the stimuli, and the other group was a nonmanipulated control group. In both experiments an attempt was made to create in the experimental group an urge to respond, either to approach (see section 5.3) or to avoid (see section 5.5). It was subsequently tested whether these manipulations resulted in an attentional bias for response-relevant stimuli. In the first study, presented in section 5.3 , a group of normal subjects was instructed to fast for 24 hours prior to the Stroop task and it was tested whether these subjects, relative to nonfasting controls, showed an attentional bias for food words. In the second study, discussed in section 5.5, the experimental subjects were instructed to suppress all thoughts about a particular topic, namely "numbers". In an earlier stuidy, presented in section 5.4, we demonstrated that such thought suppression instruction has the paradoxical effect that subjects become preoccupied with that specific thought content. In the section 5.4 experiment subjects reported that the thought they were supposed to suppress frequently intruded into consciousness, even though they made great effort to avoid thinking about it. Based on the adaptiveness hypothesis, it was expected that the urge not to think about numbers would result in an attentional bias in favour of number words.

The seven experimental studies, presented in chapter 5 , are followed by a general discussion, in which the findings of these experiments are considered and integrated. For the sake of clarity, a matrix of main hypotheses and experiments is offered alongside. The section 5.4 experiment, an investigation of the paradoxical effect of thought suppression, is not included in this matrix because it does not deal with attentional bias. The hypotheses underlying this experiment are only relevant for the section 5.5 experiment. 


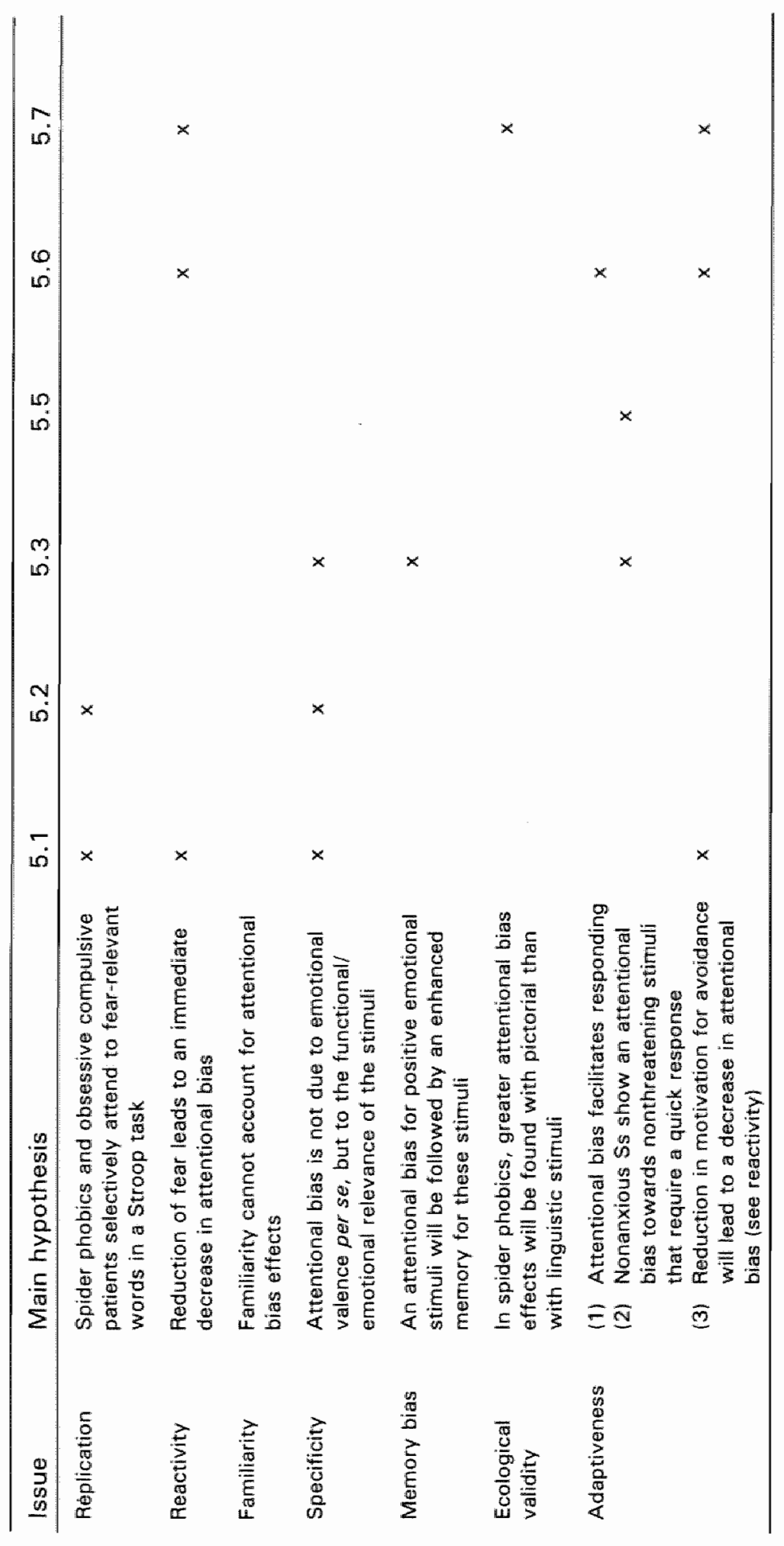


Chapter 5

Experimental data 


\subsection{Attentional bias and spider phobia: Conceptual and clinical issues ${ }^{1}$}

\section{Summary}

Experimental evidence indicates that anxious subjects show an attentional bias for threat-relevant information. Foa and McNally (1986) and Watts et al. (1986a) reported that behaviour therapy can eliminate this attentional bias. A replication study was carried out in order to increase the evidence for exposure being the crucial component in reducing attentional bias. Moreover, in this study some conceptual and clinical issues are explored. The theoretical and clinical implications of the results are discussed.

\section{Introduction}

In recent years there has been an increasing amount of research into cognitive processes in anxiety disorders. One line of experimental research has focused on attentional bias: the selective allocation of attentional resources to cues related to threat and danger. A number of laboratory paradigms have been used to investigate the attentional bias phenomenon (for an extensive review see Williams, Watts, MacLeod, \& Mathews, 1988). Various attentional bias tests have been administered to patients with different diagnoses, including social phobia (Hope, Rapee, Heimberg, \& Dombeck, 1990), panic disorder (McNally, Riemann, \& Kim, 1990b), generalized anxiety disorder (MacLeod, Mathews, \& Tata, 1986; Mathews \& MacLeod, 1985; Mathews \& MacLeod, 1986), obsessive compulsive disorder (Foa \& McNally, 1986), post-traumatic stress disorder (McNally, Kaspi, Riemann, \& Zeitlin, 1990a) and spider phobia (Watts, McKenna, Sharrock, \& Trezise, 1986a). The attentional bias demonstrated in almost every published study concerning this topic is specific for disorder-related cues, social phobics being hyperattentive to social threat cues, spider phobics to spider cues etc.

Attentional bias is not an unalterable phenomenon. Treatment studies by Foa and McNally (1986) and Watts et al. (1986a) showed that behaviour therapy can eliminate attentional bias in obsessive compulsive patients (Foa and McNally) and in spider phobics (Watts et a/.). However, both in the Foa and McNally study and in the Watts et al. study the behaviour therapy was far from a clear-cut experimental intervention. Foa and $\mathrm{McNally}$ reported that the obsessive compulsive patients received 15 daily sessions of both imaginal flooding and exposure in vivo with response prevention. Moreover, their patients performed 4 hours of unspecified daily home-

1. Lavy, E.H., Hout, M.A. van den, \& Arntz, A. 11993). Attentional bias and spider phobia: Conceptual and clinical issues. Behaviour Research and Therapy, 31,17 24. 
work between the sessions. Watts et al. offered their spider phobics four sessions of "desensitization in vivo" in small groups, spread over 6-8 weeks. The authors did not report what happened in these sessions, only that there was an emphasis on handling the spiders as much as possible and that the least phobic individuals served as models. However, the avoidance test failed to show significant improvement in minimum distance to the spider. Thus, the reduction in attentional bias does not seem to have been caused by a reduction of fear.

Although studies have been performed that focus on the effect of treatment on attentional bias, one was never told exactly what it was that happened during these therapy sessions or, what is perhaps even more important, what happened berween these sessions, out of sight of the researchers. While the authors suggest that exposure is the component in treatment that is crucial for the elimination of attentional bias, this suggestion has yet to be more directly tested. Providing such test was the first aim of the present study.

The presence of attentional bias in anxious patients and its possible reduction by exposure may best be conceptualized in terms of the "information structure" theory described by Foa and Kozak (1986). According to this theory, emotions are represented as a network in memory structures. These structures are not limited to actual information about stimuli and responses but also include interpretations about their meaning for the individual. In the case of fear these structures serve as a programme for escape and avoidance behaviour (Foa \& Kozak, 1986). Hyperattention to fear-associated stimuli may lead to a facilitated triggering of the fear network by these stimuli. When a fear-associated stimulus is detected, processing resources are automatically diverted from less salient cues to these feared stimuli in order to escape the danger as quickly as possible. Hyperattention to feared stimuli can, therefore, facilitate an early escape.

Fear structures are hell to be modifiable to the extent that a patient is confronted with information that is incompatible with these interpretative structures. Processing of incongruent information results in disintegration of the old structures and allows for the formation of new structures (Foa \& Kozak, 1986). When the fear network has disintegrated, the stimuli and fear structures are not linked anymore. In the process, the basis for hyperattention disappears and attentional bias is reduced. What is crucial is that during exposure, the fear network is completely activated and the incompatible information is optimally processed. In clinical practice anxious subjects sometimes appear to suboptimally process information about the feared stimuli, possibly because they use cognitive avoidance strategies like distraction, dissociation or concentration on nonfearful elements of the feared stimuli ${ }^{2}$. As a result of these strategies, exposure might be less effective in disintegrating the fear network and the reduction in attentional bias will be smaller. The implication is that an exposure treatment in which cognitive avoidance is prevented will result in a larger attentional bias reduction than an exposure without such explicit prevention.

Because of its theoretical significance, we decided to try to replicate the attentional bias reduction, due to exposure in vivo, with a very discrete intervention that is still clinically relevant: a one-session treatment of spider phobia as described by 
Ost (1989). In order to investigate whether prevention of cognitive avoidance results in a superior attentional bias reduction, two treatment conditions were used (more detaled information about the treatments is given in the Method section).

Two other objectives were added to this experiment. Firstly, the most extensively used paradigm in attentional bias studies is the modified Stroop task. The interference index, the measure of attentional bias, is always calculated by subtracting the response latencies for colour naming neutral words from those for colour naming phobia-relevant words. However, in order to control for negative emotional value, it appears more pertinent to determine the relative deterioration in colour naming by calculating the interference between phobia-relevant and general negative words. As a matter of fact, in some studies (e.g. Watts et al., 1986a; Williams \& Broadbent, 1986), general negative words are used in the test, but merely to demonstrate that anxious subjects do not show an attentional bias towards those words. In the present study, both types of interference indlices (phobia-relevant words vs. neutral and general negative words) were calculated and analyzed.

A last point of interest was the association between attentional bias and other anxiety measures, such as self-reported fear and behavioural approach. Insight into these associations could be especially valuable if measures of attentional bias are to be used to diagnose anxiety disorders or to evaluate treatment effects. Thus, the final goal of this experiment was to investigate whether attentional bias is correlated with overt avoidance behaviour and self-reported fear.

\section{Method}

\section{Subjects}

Thirty-six spider phobics (all female, mean age 31.4 years) participated in the experiment. They were recruited through articles in local newspapers in which the treatment of spider phobia was explained and an opportunity to take part in the "exposure researrch programme" was offered. The same information was given in a programme on satellite television. Subjects met the DSM-III-R criteria for simple phobia. For most subjects, the age of onset was early childhood. Subjects received free treatment in exchange for their participation in the research project.

A control group was added for two reasons: (1) to investigate whether attentional bias towards spider cues is specific to spider phobics, which seemed relevant because a new interference index was used, and (2) as a check for test-retest effects, which was important since the two Stroop tests were presented within a few hours of each other. Like the spider phobics, the control subjects performed the Stroop test twice, with a 2 hour interval between these tests. During this period the nonanxious control subjects left the laboratory. Thirty female controls (matched for age, mean age 34.1 years, and level of education with the spider phobics) were recruited via posters in supermarkets, in halls of blocks of flats and in a fitness centrum and through house-to-house distribution of a letter in which women were asked to take part in experimental research. Confirmation was made by telephone that the subjects were not afraid of any kind of animal (including spiders). After performing the second Stroop test, the control subjects completed the Spider Phobia Questionnaire as a check on the absence of spider phobia. 


\section{Assessment}

Spider Phobia Questionnaire (SPO). In order to measure subjective anxiety with regard to spiders the Spider Phobia Questionnaire (SPQ) Was used (Klorman Weerts, Hastings, Melamed, \& Lang, 1974). The SPQ consists of 31 statements of self-reported fear that have to be answered with "yes" or "no". Phobic subjects completed this questionnaire twa times, once before and once after treatment. Nonphobic controls filled in only one SPQ.

Behavioural Approach Test (BAT). The spider phobics participated in a behavioural approach test (BAT). During this test, subjects were seated in a chair behind a large table $(0.4 \mathrm{~m} \times 3.0 \mathrm{~m})$. At the far end of the table, a live spider (Tegenaria Atrica), about $3 \mathrm{~cm}$ long was kept in a closed glass jar. A guided string was fastened to the jar and subjects were instructed to pullil the spider as nearby as they would normally tolerate. The BAT was scored on a 13-point scale from 0 (the glass jar is not moved at all, the distance is still $3 \mathrm{~m}$ ) to 12 (the spider is on the subject's handh.

\section{The Stroop test}

Stroop words were presented to the subject via an Olivetti M250 microcomputer with colour monitor. A Sony Cardoid Dynamic microphone F560, which the subject held in her hands, was connected to a voice level detector that stopped the computer's clock at the initiation of the subject's vocal response. The computer reconded these response latencies per word in milliseconds. Errors were marked with the use of an interface button, operated by the experimenter.

All words appeared in the centre of the computer screen. A little fixation cross appeared at the centre $2000 \mathrm{msec}$ before word onset. Words appeared in $8 \mathrm{~mm}$ block letters in red, blue, yellow or green. The appearance of both words and colours was random; however, during the test each word was presented four times and the same colour could not occur more than twice in succession. The word was on the screen until the subject reacted but for no less than 1500 msec and for no more than $3000 \mathrm{msec}$. If the voice level detector did not record a response latency within $3000 \mathrm{msec}$, the trial was considered as missing and an error was registered.

There were three types of stimulus words: 14 spider words, 14 general negative words and 14 neutral words. Each word was presented four times resulting in a 168 item Stroop test. Some of the spider words were taken from the Watts et al. experiment; others were generated by the authors.

For the general negative and neutral words, a list was constructed with 100 words, 50 negative and 50 neutral. Ten subjects, who did not know about the experimental aims of the study, rated the words on a 10 -point scalle $11=$ very nega. tive, $5=$ neutral, 10 =very positive). Taking into account the length of the words and the number of syllables, the 14 best fitting negative (mean $=2.2$ ) and neutral (mean $=5.1$ ) words were chosen. There was no overlap in the ratings of the neutral and negative words. Table 5.1 .1 shows the stimulus words used, translated into English.

\section{Treatment}

The treatment, which lasted 2.5 hours, was individually given and the phobics were exposed to live spiders. The therapist could make a choice out of at least 30 different sizes and types of spiders. A typical treatrnent session started with a small spider in a closed glass jar. The subject would gradually attempt more "daring" 


\begin{tabular}{lll}
\hline Spider words & Negative words & Neutral words \\
\hline spider & hate & fork \\
web & bomb & chin \\
creep & abuse & square \\
legs & violence & percent \\
hairy & war & spindrier \\
poisonous & torture & coatpocket \\
suddenly & deceit & potato \\
quickly & murder & plate \\
fright & disaster & month \\
itching & mutilation & owner \\
insect & lonely & key \\
hidden & hunger & context \\
cobweb & fight & mineral \\
obscured & wound & pencil \\
\hline
\end{tabular}

Table 5.7.7: Stimulus words used in the Stroop test

tasks with the spider, the last of which would be letting it walk around on her hands. Intermediate tasks included, for instance, having the spider walk in an open basin or on a ruler and having the phobic touch it with a pencil or her finger. After succeeding at all tasks, the subject progressed to a more difficult (i.e. larger, scarier) spider and the exposure to the hierarchy of tasks was repeated.

There were two treatment conditions. In one condition, cognitive avoidance was prevented/discouraged by stimulating the patient to elaborate as much actual information about the spider as possible. Subjects in the elaboration condition were continuously encouraged to pay full attention to the objective features of the spider and its behaviour, to describe these to the therapist and to perform small behavioural experiments with the spider. By means of this forced elaboration, the attention of the subject was focused on realistic information. In the nonelaboration condition, emphasis was placed completely on performing the exposure exercises; all kinds of vaboration were discouraged (see Arntz and Lavy (1993a) for more detailed information about the treatment and its effects). Subjects were randomly assigned to either the elaboration or the nonelaboration exposure condition.

\section{Procedure}

The spider phobics completed the SPO upon arrival. All subjects were then introduced in a dimly lit room and were instructed about the Stroop test. They were given ten practice trials with the words ONE, TWO. THREE, FOUR and FIVE in order to get used to the procedure. Then subjects did the experimental Stroop test.

After the Stroop test, the spider phobics carried out the BAT and were given a one-session treatment, either an elaboration or a nonelaboration exposure. After treatment, the subjects again did the Stroop test and the BAT and filled in the SPQ for the second time.

The nonphobic control subjects were retested after a 2 -hour period. They completed the SPQ after the second Stroop test and were then informed about the aims of the experiment. 


\section{Results}

\section{Checks before analyzing the Stroop data}

Before treatment, the phobic group had higher SPO scores than the nonanxious control group $[t=25.78, P<0.001]$. Moreover, the mean SPQ score of the phoblc group $\left(m=23.19, s_{n} \mathrm{~d} .=3.21\right)$ before treatment was comparable with the mean score of spider phobics from the Fredrikson (1983) study $(\mathrm{m}=23.76, \mathrm{~s} . \mathrm{d}=3.80$ ). There was no overlap between the SPO scores of the phobic and those of the control group. Taken together, the composition of the two groups was adequate.

Two measurements were used in order to determine whether the exposure treatment of the phobic subjects had been effective: the SPQ and the BAT. The SPQ scores declined dramatically $[\mathrm{t}(35)=14.64, P<0.0011$ and the BAT scores improved in a similar fashion $[t(35)=-10.52, P<0.001]$; for detailed information about the treatment effects see Arntz and Lavy (1993a). Thus, the one-session exposure treatment was successful.

To verify whether the randomization procedure with the phobic subjects was effective, we studied whether subjects in the elaboration and nonelaboration condition differed in their fear of spiders before treatment. We found no difference in SPQ scores $[t(34)=0.46, P=0.647]$ or in BAT scores $[t(34)=0.54, P=0.591]$.

To check whether the elaboration exposure had indeed enhanced information processing, phobic subjects were given a free recall test. They were asked to write down everything they remembered about every spider that had been used in the exposure session. As expected, more details were remembered by subjects in the elaboration exposure condition (mean $=5.9$ ) than by subjects in the nonelaboration exposure condition (mean $=4.5)$; this difference was significant $[t(34)=1.75$, $P=0.045$, one-tailed].

\section{Stroop data}

Before analyzing the Stroop data, errors were excluded from analysis. Errors ( $<2 \%$ of the trials) were either marked by the experimenter or indicated by response latencies less than $300 \mathrm{msec}$ or more than $3000 \mathrm{msec}$. Phobic and nonanxious subjects did not differ in the number of errors they made $[t(64)=0.32, P=0.750]$.

\section{Before treatment}

In order to differentiate between the various types of interference indices, two spider interference indices were calculated: (1) spider versus neutral interference and (2) spider versus general negative interference. One-tailed t-tests revealed that subjects in the phobic group had significantly larger spider interference indices than subjects in the control group, irrespective of whether the response latencies for spider words were compared with neutral words $[t(64)=3.43, P<0.001]$ or generall negative words $[\mathrm{t}(64)=3.19, P=0.001]$. Moreover, in the phobic group the two types of spider interference indices did not differ significantly $\| \mathrm{t}(35)=1.46$. $P=0.1541$ and were highly correlated $(r=0.901)$. There was no evidence that the spider versus general negative interference index was superior to the more commonly used spider versus neutral index. Because the same results were found, irrespective of the interference index used, in subsequent analyses the average of the two spider interference indices was used.

The 168 trials were divided into four blocks of 42 trials each. For each block, a mean response latency and mean spider interference index was calculated for each 
subject and these variables were analyzed by means of MANOVA trend analyses. The mean response latency and mean spider interference index, as well as the linear trends of these variables, were analyzed. There appeared to be a multivariate between-group effect $[F(4,61)=5.14, P=0.001]$. Univariate $F$-tests showed that the phobic and control group differed significantly in mean response latency $[F(1,64)=13.17, P=0.001$, mean phobics $=768$ msec, mean controls $=667$ msec $]$ and mean spider interference index $[F(1,64)=11.74, P=0.001$, mean phobics $=39$ msec, mean controls $=-3$ msecl. The two groups did not differ in linear trends of response latency $[F(1,64)=0.00]$ or spider interference index $[F(1,64)=0.27$, $P=0.61]$. Moreover, for neither response latency $[F(1,64)=0.10, P=0.75]$ nor spider interference index $[F(1,64)=2.15, P=0.15]$ was there a main linear trend. Thus, there was no evidence for habituation or sensitization during the Stroop test (see also figure $5.1,1$ ).

A final t-test showed that the phobic and control groups did not differ in general negative versus neutral interference $[t(64)=1.11, P=0.27]$. Thus, the attentional bias of the phobics was selective for spider words.

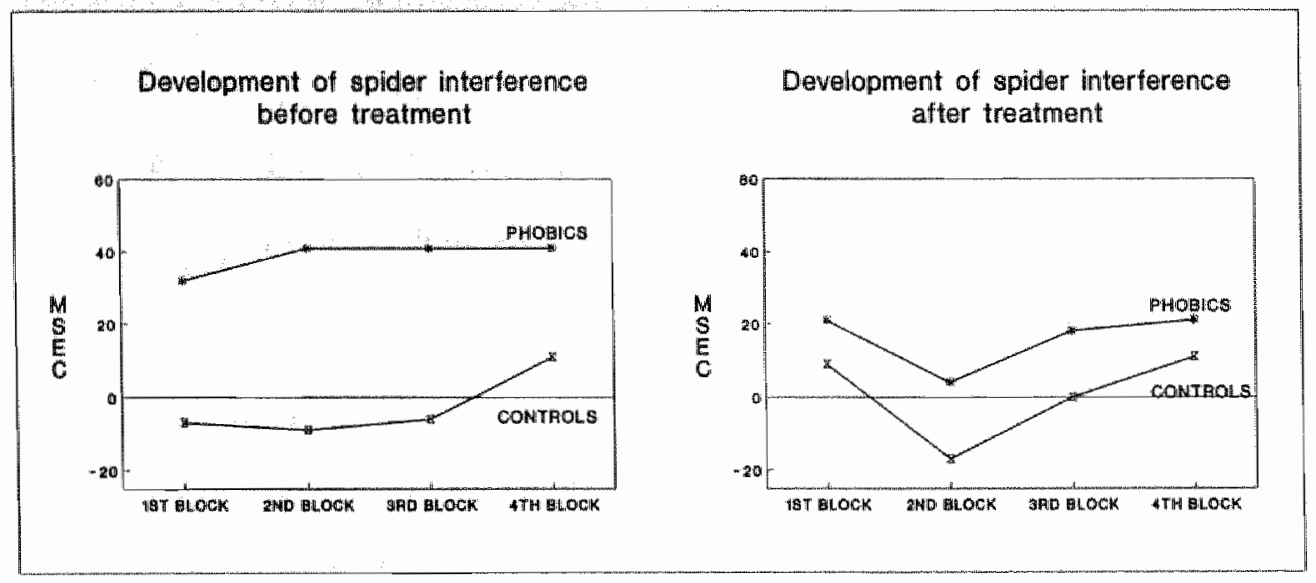

Figure 5. 1.1: Development of spider interfenence during the Stroop test

\section{After treatment}

After treatment (and for the control group, after the waiting period), the data were again divided into four blocks of 42 trials each. For each block the mean response latency and mean spider interference index were calculated and submitted to MANOVA trend analyses. The mean and linear trend were analyzed. The multivariate between-group effect was not significant, but a trend was shown $[F(4,61)=$ 2.39, $P=0.15 \mathrm{j}$. Univariate F-tests showed that the phobic and control group still differed significantly in mean response latency $[F(1,64)=6.39, P=0.014$, mean phobics $=762$ msec, mean controls $=678 \mathrm{msec}]$ and mean spider interferencie index $[F(1,64]=5.90, P=0.018$, mean phobics $=16$ msec, mean controls $=1 \mathrm{msec}]$. Again, there were no group differences in linear trends of response latency $[F(1,64]=2.15, P=0.15]$ or spider interference index $[F(1,64)=0.62, P=0.431$, as can be seen in figure 5.1.1. 


\section{Treatment effect on attentional bias}

Treatment effect was demonstrated by the decrease in spider interference index in the phobic group $[t(35)=2.44, P=0.02]$. The spider interference index in the control group did not change significantly $[t(29)=0.69, P=0.50]$. The decrease in the phobic group was significantly higher than in the control group $\mid F(1,64)=5.46$, $P=0.02$ ). There were no other group differences due to treatment effect (see figure 5.1.2). Except for spider words, in the phobic group, there were no test-retest differences in mean response latencies.

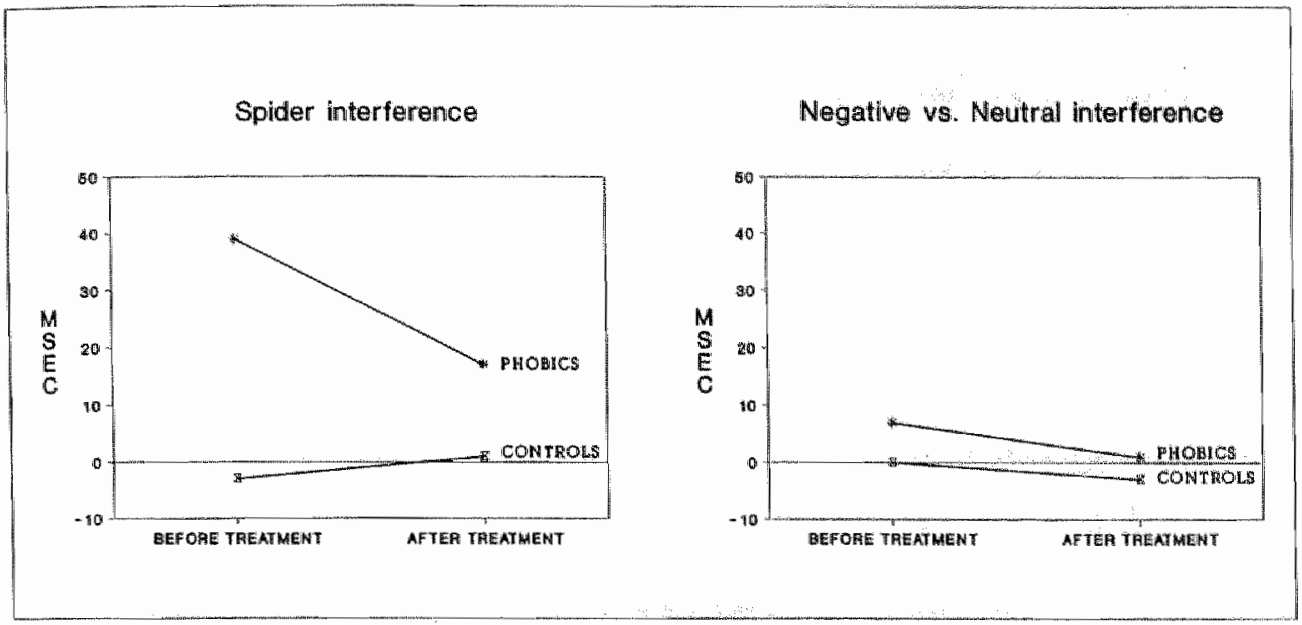

Figure 5. 1.2: Mean interferences before and after treatment

\section{Elaboration/nonelaboration}

For these analyses only data from the phobic group were available, because the control group did not receive any treatment. Data were transformed into mean spider interference index for each block. Again, the mean and linear trend were analyzed with MANOVA trend analyses. These analyses revealed that neither before $[(F[2,33)=0.89, P=0.42]$ nor after treatment $[F(2,33)=0.49, P=0.62]$ there was any difference in mean interference index or linear trend. The decrease in spider interference index did not differ between the two conditions $[F(1,34)=0.53$. $P=0.471$. Therefore, there is no evidence that the elaboration manipulation enhanced disintegration of the fear network.

\section{Correlations between attentional bias and other measurements}

Only the data obtained from phobic subjects were analyzed to avoid "false" correlations due to a bimodal distribution of the data. To investigate whether the spider interference index is associated with the SPQ and BAT scores, two Pearson's correlations were calculated for both the pre- and the post-treatment data. Before treatment, neither SPQ nor BAT correlated significantly with the spider interference index $\left(r_{\mathrm{BAT}}=-0.15, r_{\mathrm{SPO}}=0.19\right)$, although the correlations were in the expected direction. Hence, spider interference index correlates positively with the self-reported 
fear and negatively with approach behaviour but does not share much variance with these measurements. After treatment, there was no correlation between spider interference and SPQ $(r=0.02)$ and only a small correlation between spider interference and $B A T(t=0.23, P=0.087)$.

A decrease in spider interference index, due to treatment, was associated with an improvement in approach behaviour $\left(r_{B A T}=-0.32, n=36, P=0.028\right)$ but not with a decrease in self-reported fear as measured by the SPQ $\left(r_{\mathrm{sPO}}=0.06, n=36\right)$. In order 10 see whether the spider interference index before treatment predicts treatment effect as measured by BAT and SPQ, correlations between the spider interference index before treatment and improvements on SPO and BAT were calculated. There was a small correlation of pretreatment spider interference index with enhanced BAT change scores $\left(r_{\mathrm{BAT}}=-0.25, n=36, P=0.069\right)$ but not with a decrease in selfreported fear $\left(r_{\text {spa }}=0.06\right)$.

\section{Discussion}

There were two main differences between the phobic and control groups. Firstly, phobic subjects reacted more slowly than the controls on all stimull. This difference between anxious and nonanxious subjects is consistent with previous findings (e.g. Mathews \& MacLeod, 1985; McNally et al., 1990a). It is unclear whether this general slowness reflects a stable trait, characteristic of anxious patients or, alternativeIy, a state phenomenon. Note that our subjects, at the first test, knew they were about to engage in massive exposure treatment, while at the post-test, after treatment, they may have been rather tired.

Although it is interesting to speculate about the backgrounds of this general slowness, what is more important in the present context is that before treatment spider phobics showed an attentional bias towards spider words. This attentional bias was reduced, but not completely eliminated, by a one-session exposure treatment. These findings are an encouraging replication of the Foa and McNally (1986) and Watts et al. (1986a) studies. The change in attentional bias does not seem to have resulted from learning effects on the Stroop per se. Both before and after treatment, the attentional bias did not decline during the Stroop task. Secondly, the decline between the first and second Stroop tasks could be completely attributed to the treatment effect because only the spider interference index in the phobic group changed over time. By using a one-session treatment, several confounding factors were ruled out; hence, there is more evidence now that exposure therapy is the crucial component in reducing the attentional bias.

Is there any evidence that the spider versus general negative words interference is a more appropriate measure of attentional bias than the ordinarily used spider versus neutral words interference? There appeared to be no difference between these two interference indices; as a matter of fact, the correlation was extremely high.

The assumption that elaboration enhances the attentional bias reduction could not be confirmed. Before rejecting this hypothesis, it may be noted that it proved extremely difficult to prevent the "nonelaboration" group from elaborating. An indication that the therapists were not very successful in carrying out exposure therapy and preventing elaboration at the same time is the finding that, although the two exposure groups differed in the number of spider details remembered, the difference 
was very small. Hence, it is doubtful whether the manipulation was successful: enough to result in a differential disintegration of the fear network. It would be interesting to replicate this study with an adjusted manipulation, in which steps are taken to reduce, if not totally prevent, elaboration in the nonelaboration group more effectivelly than in the present study.

Might it be worthwhile using attentional bias measurements in the diagnostic process or in therapy evaluation? As it comes to clinical classificatory diagnosis. there appears to be no practical need for attentional bias measures. At least as far as simple phobias are concerned, classification is generally no problem for the clinician and attentional bias measures would then be a method too circumstantial. Moreover, our attentional bias measures show very weak correlations between subjective anxiety and avoidance behaviour on the one hand, and attentional bias on the other. Apparently, attentional bias measures tap different aspects of the fear system. To the degree that attentional bias assesses an aspect of how information is processed while subjective and behavioral measures assess the result of this processing, attentional bias measures may make an independent and relevant contribution to prognosis. To our disappointment, we found only a marginal correlation between pretreatment attentional bias and treatment success. Still, there is a distinct possibility that the attentional bias phenomenon is clinically relevant. For instance, relapse after successful exposure treatment is not uncommon. To the degree that attentional bias measured during or at the end of treatment reflects residual intactness of the fear network, one should be able to decide by means of attentional bias measures whether further treatment is indicated, c.q. if relapse is likely. In arder to explore this issue, we sent the spider phobics a SPQ as a 1-year follow-up. Unfortunately, the response rate was too small for us to draw conclusions. More research on the relapse-predicting potential of attentional bias measures is, therefore, warranted.

Apart from investigating whether attentional bias may have prognostic or evaluative utility, it is important to know what role attentional bias plays in anxiety disorders. It seems plausible to presume that attentional bias enhances anxiety, because by paying relatively much attention to threatening information, the anxious individual experiences his environment as too dangerous. The hypothesis of Eysenck and Mathews (1987b) corresponds with this opinion. They suggested that individuals high in trait anxiety tend to have a preattentive selective bias that leads them to allocate processing resources to threatening stimuli. This selective processing results in elevated state anxiety levels and may predispose them to anxiety disorders. While it may seem evident that attentional bias increases anxiety, there is no experimental evidence to support this suggestion. On the other hand, there is some experimental evidence that attentional bias is a consequence of anxiety. In a recent study (Merckelbach, van Hout, de Jong, \& van den Hout, 1990b) it was demonstrated that attentional bias can be provoked in nonanxious subjects by means of aversive classical conditioning. Discussing the results, Merckelbach et al suggested that clinical attentional biases may originate from classically conditioned fear as well. The present data do little to clarify the causal status of attentional bias. The fact remains that for both clinical and theoretical reasons, rigorous experimental analysis of the causality issue is badly needed. 


\title{
5.2 Selective processing of emotional information in obsessive compulsive disorder ${ }^{3}$
}

\begin{abstract}
Three possible explanations for attentional bias effects in anxious subjects have been formulated: the threat relatedness hypothesis, the emotionality hypothesis of Martin et al. (1991) and the concern-relatedness hypothesis of Mathews and Klug (1993). In order to investigate these three hypotheses, an experiment was carried out with 33 obsessive compulsive $10 \mathrm{Cl}$ patients and 29 normal controls. Both groups colour named a Stroop card with 5 word sets: noutral words and four emotional word sets (a $2 \times 2$ matrix of words, related/unrelated to obsessive compulsive disorder and positively/negatively valenced).

In line with previlous studies, OC patients selectively attended to negative OCrelated cues; this supports the threatirelatedness hypothesis. Although the set-up of the experiment was similar to the Mathews and Klug (1993) study, no evidence was found for the concern-relatedness hypothesis, i.e. the $O C$ patients did not show an attentional bias for positive OC-related words. Two possible reasons for these contradicting findings are discussed.
\end{abstract}

\section{Introduction}

Although idiosyncratic attentional biases in favour of threatening information are frequently shown in most anxiety disorders for a review see Williarns, Watts, MacLeod, \& Mathews, 1988), relatively few studies have addressed the issue of attentional bies in obsessive compulsive (OC) patients (Foa, llai, McCarthy, Shoyer, \& Murdock, 1993; Foa \& McNally, 1986). The results of the attentional bias studies are consistent with several important cognitive theories, like the emotional network theory of Bower (1981; 1987 ) and Beck's theory of anxious people suffering from over-active danger schemata (Beck \& Emery, 1985). Until recently, the threat-relatedness hypothesis was advocated without doubt by attentional bias researchers.

But Martin, Williams and Clark (1991) criticized the current attentional bias studies and argued that threat was confounded with emotionality. Using a Stroop paradigm, they demonstrated that anxious subjects not only showed an attentional bias to threatening stimuli, but also to pasitive stimuli which were as emotional as the threatening words. According to Martin et al. 11991), the threat-relatedness hypothesis had to be replaced by the emotionality hypothesis, since anxious sub-

3 Lavy, E, Oppen, P. van, \& Hout, M. van den (1993). Selective processing of emotional information in obsessive compulsive disorder. Behavour Research and Therepy (in pross). 
jects do not only show an attentional bias for threatening stimuli, but attend to emotional material in general.

However, as Mathews and Klug (1993) remarked properly, several positive words Martin et a/. (1991) used, were exact opposites of anxiety and it could be argued that antonyms of threatening words are indirectly, via theilr threatening opposites, also associated with anxiety. It therefore remained unclear whether anxious subjects show only an attentional bias for cues associated with their personal concerns or for emotional cues in general.

In order to clarify this issue, Mathews and Kllug (1993) carried out an experiment with anxious patients with diverse diagnoses. They constructed five sets of words to be collour named: anxiety-related and -unrelated positive words, anxiety-related and -unrelated negative words and emotionally neutral words; as judged by a panel of professionals. The results of their study suggested that anxious subjects, compared with normal controls, do not show an attentional bias for emotional words which are unrelated to anxiety, but attend selectively to both positive and negative anxiety-related stimuli. Consequently, they rejected the emotionality hypothesis of Martin et al. (1991) and concluded that anxious subjects show an attentional bias for stimuli which are semantically associated with their emotional concerns. A third explanation had thus been proposed: the concern-relatedness hypothesis.

In the same period, we started a similar experiment with $O C$ patients. Results from former experiments (Foa et al., 1993; McNally, Kaspi, Riemann, \& Zeitlin, 1990a) suggested that the attentional bias effect in OC patients is highly specific and only shown for idiosyncratic cues. Therefore, we constructed two versions of the experimental Stroop test: one test for patients with washing rituals and another for patients with checking rituals. The aim of the study was to find out for which of the three hypotheses evidence was found.

\section{Method}

\section{Subjects}

Thirty-three $O C$ subjects participated in the experiment before entering a treatment programme in the Amsterdam Psychiatric Centre. All subjects met the DSM-III$R$ (APA, 1987) criteria for obsessive compulsive disorder (OCD) lasting for at least one year. All subjects were screened by a Dutch translation of the standardized Anxiety Disorder Interview Schedule (ADIS: Bouman, Scholing, Emmelkamp, \& Dijkstra, 1987), conducted by a trained psychiatrist. The OCD thad to be the main problem. All subjects were outpatients. On behalf of the experiment, they were classified as washers $(n=13)$ or checkers $(n=20)$, according to their dominant avoidance behaviour. Twenty-nine normal controls were matched with the patient group for gender, age and level of education. None of the control subjects had a history of anxiety disorder.

\section{Stroop words}

The OC words were derived from a pilot test in which six OC patients, who did not participate in the experiment, scored 95 OC-related items. They were asked to decide (1) how much the word was related to their obsessive compulsive complaints $[1=$ not at all related, $10=$ fully related] and (2) what the emotional valence of the word was $[1=$ extremely negative, $10=$ extremely positive]. Three pilat 
subjects were diagnosed as washers, the other three as checkers. For both washers and checkers the eight most $O C$-related positive and negative words were chosen. As can be seen in table 5.2.1, there was some overlap in the choice of the two diaginostic groups. The mean OC-relatedness scores were 8.4 (positive OC-words) and 8.9 (negative $O C$-words); the corresponding mean emotional valences were resp. 8.8 and 2.0 . Thus, the OC words were strongly linked with obsessive computsive complaints and were extremely emotional. There were no significant differences between washers and checkers.

The neutral, positive and negative emotional words were derived from another pilot study in which 12 normal controls, who did not participate in the experiment, rated the emotional valence of 150 items, using the same emotionality rating as the six OC patients. The mean emotional valences were 1.8 (negative words), 5.1 (neutral words) and 8.4 (positive words).

There were no significant differences between the emotionality scores of the OCrelated words and unrelated positive and negative words. The seven word sets were matched for number of syllables and total length in the Dutch language. The word sets are presented in table 5.2.1.

\section{Stroop test and procedure}

Words were presented on a white card $(30 \times 21 \mathrm{~cm})$ and were plotted in $4 \mathrm{~mm}$ red, blue, yellow or green block letters. Words were plotted in rows of eight words from the same word set. The order of presentation was: Neutral row, Negative Emotions row, Positive Emotions row, Positive $O C$ row and Negative $O C$ row. This sequence was repeated twice, making a total of 10 rows. The order of presentation minimized potential carry-over effects, whille any practice effect would tend against the expected retardation effects.

Subjects were individually tested. They were instructed to name the colours as quickly and accurately as possible, and to ignore the meaning of the words. To prevent the OC patients from dwelling upon their faults, the subjects were emphasized that they did not have to correct any errors but simply had to continue the test. In order to get accustomed to the routine, subjects were firstly given a practice Stroop card. After the practice test, OC patients were presented with either the washers ( $n=13$ ) or the checkers $(n=20)$ Stroop card, in conformity with their dominant avoidance behaviour. Thirteen controls were presented with the washers Stroop card, sixteen with the checkers card. The colour naming times of the subjects were audio recorded on U-matic tape and assessed afterwards.

\section{Apparatus and measurements}

In order to determine the response times, a computer clock (Avitel generator TGE 2040 ) was appended to the audio responses. A JVC CR 8250 editing recorder was used for scoring the U-matic tapes. Colour naming times were determined per row; the computer clock, which had an accuracy of $2 / 100 \mathrm{sec}$, was used to decide when the focal response to the last stimulus of a row was faded out completely. Errors, that is naming of a wrong colour or occasionally reading the word, were registered. 


\begin{tabular}{|c|c|c|c|c|c|c|}
\hline Neutral & Negative & Positive & $\begin{array}{l}\mathrm{OC} \\
\text { Positive } \\
\text { washers }\end{array}$ & $\begin{array}{l}\text { OC } \\
\text { Negative } \\
\text { washers }\end{array}$ & $\begin{array}{l}\text { OC } \\
\text { Positive } \\
\text { checkers }\end{array}$ & $\begin{array}{l}\text { OC } \\
\text { Negative } \\
\text { cheokers }\end{array}$ \\
\hline $\begin{array}{l}\text { square } \\
\text { fork } \\
\text { coatpocket } \\
\text { potato } \\
\text { percent } \\
\text { month } \\
\text { blanket } \\
\text { key }\end{array}$ & $\begin{array}{l}\text { war } \\
\text { hate } \\
\text { treachery } \\
\text { torture } \\
\text { violence } \\
\text { deceit } \\
\text { abuse } \\
\text { murder }\end{array}$ & $\begin{array}{l}\text { love } \\
\text { friend } \\
\text { happy } \\
\text { trust } \\
\text { felicity } \\
\text { humor } \\
\text { fun } \\
\text { party }\end{array}$ & $\begin{array}{l}\text { tidy } \\
\text { clean } \\
\text { healthy } \\
\text { newt } \\
\text { cleanly } \\
\text { pure } \\
\text { protected } \\
\text { safe }\end{array}$ & $\begin{array}{l}\text { filthy } \\
\text { dirty } \\
\text { fail } \\
\text { mess } \\
\text { doubt } \\
\text { uncertain } \\
\text { disease } \\
\text { guilt }\end{array}$ & $\begin{array}{l}\text { precise } \\
\text { assured } \\
\text { relaxed } \\
\text { precaution } \\
\text { perfect } \\
\text { scheme } \\
\text { protected } \\
\text { safe }\end{array}$ & $\begin{array}{l}\text { latal } \\
\text { disaster } \\
\text { fail } \\
\text { wrong } \\
\text { doubt } \\
\text { uncertain } \\
\text { guilty } \\
\text { grief }\end{array}$ \\
\hline
\end{tabular}

Table 5.2.1: Stimulus words

\section{Results}

OC patients and normal controls did not differ in the number of errors they made $[\mathrm{t}(60)=0.63$, n.s.], in both groups errors were extremely rare (less than $1 \%$ of the responses).

Colour naming times per row were submitted to a threeway Group Ipatient, control) $x$ Sequence (row 1-5, row 6-10) $\times$ Word type (Neutral, Negative, Positive, OC Positive, OC Negative) ANOVA with repeated measures. Due to recording fallure the data of one control subject were incomplete, leaving 61 subjects for analysis. The Sequence $[F(1,59)=53.75, P<0.001]$ and Word type main effect $[F(4,236)=6.22$, $P<0.001]$ were significant, referring to a general slowing down as the test progressed and overall differences between the five word types. As can be seen in table 5.2 .2 , responses were most rapid for neutral rows and most delayed for OC-related negative rows. Since neutral and OC-related negative rows were respectively the first and last row in order, the Sequence $x$ Word type interaction was also significant $[\mathbb{F}(4,236)=26.16, P<0.001]$. The Group main effect was not significant $\left[F(1,59)=0.06, n_{.}.\right]$, but the crucial Group by Word type was clearly significant $[\mathrm{F}(4,236)=3.37, P=0.011]$. The remaining two- and threeway interactions were not significant.

\begin{tabular}{lll}
\hline & OC patients & Normal controls \\
\hline neutral & $5.69(1.06)$ & $6.00(1.32)$ \\
positive emotions & $6.34(1.37)$ & $6.30(1.06)$ \\
negative emotions & $6.14(1.29)$ & $6.10(1.22)$ \\
positive OC & $5.81(1.22)$ & $6.24(1.32)$ \\
negative OC & $6.55(1.22)$ & $6.19(1.00)$ \\
\hline
\end{tabular}

Table 5.2.2: Mean response times (sec), standard deviations in parentheses 
In order to interpret the significant Group by Word type interaction effect, interference scores were calculated for the four emotionally valenced word types. The iriterference scores were computed by subtracting the latencies for neutral words from the latencies for each of the emotional words. As can be seen in figure 5.2.1, four separate t-tests (with alpha adjusted to the Bonferroni inequality, $\propto=0.013$ ) revealed that the patient and control group did nat differ significantly on either of these interferences, except the OC Negative interference [t $(59)=2.57, P=0.013]$. The lack of significance was especially prominent for the interference of $\mathrm{OC}$-related positive cues. Thus, in contrast with the Mathews and Klug (1993) study, we did not find an attentional bias for positive $O C$ words. Since there was a nonsignificant trend for $O C$ patients to delav on both unrelated emotional word sets, we averaged the interferences for unrelated positive and negative words and tested whether $O C$ patients generally attended more to unrelated emotional words than normal controls. The t-test provided some evidence for the emotionality hypothesis $[\mathrm{t}(59)=1.93, P=0.059]$, but the results did not reach significance. Hence, although their was a trend for OC subjects to show a greater attentional bias in favour of OCunrelated emotional stimuli than normal controls, this difference was not significant. Insufficient evidence was thus found for either the concern-relatedness hypothesis of Mathews and Klug (1993) or the emotionality hypothesis of Martin et al. (1991).

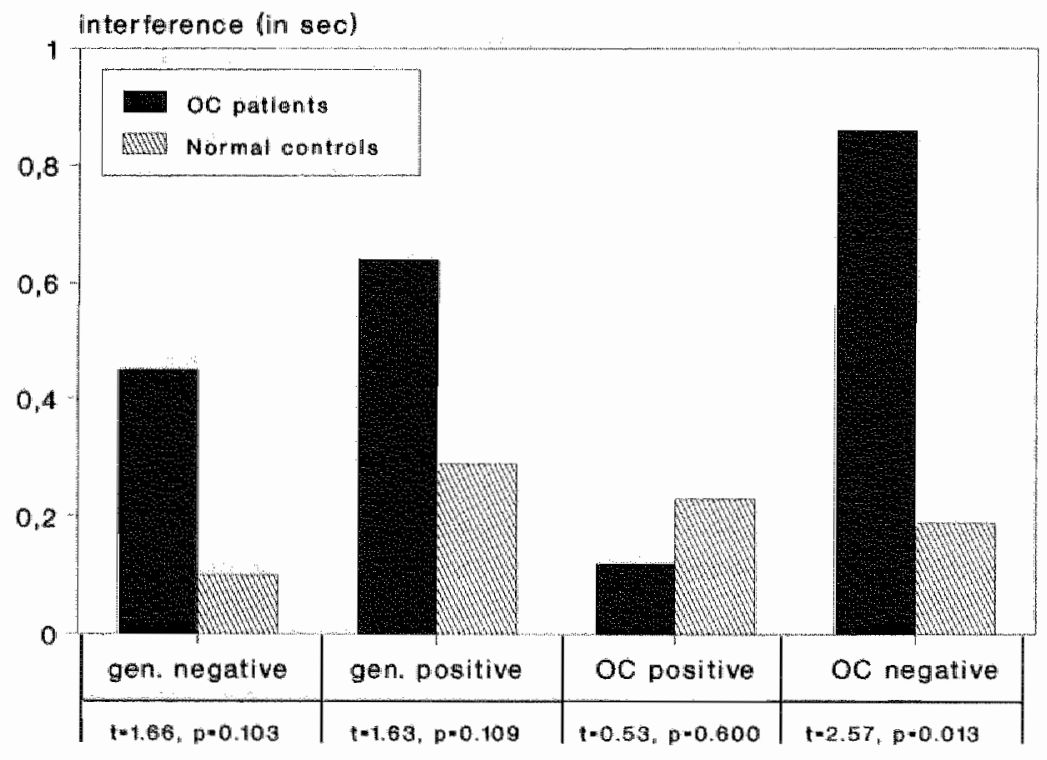

Figure 5.2.1: Mean interference scores (sec), tested for significant differences between the two groups 


\section{Discussion}

In recent years, three possible explanations for attentional bias effects in anxious subjects have been formulated: the threat-relatedness hypothesis, the emotionality hypothesis and the concern-relatedness hypothesis. Results of the present study only provide clear evidence for the first and most restricted explanation, i.e. anxious subjects selectively attend to threatening stimuli associated with their fears. Since the $O C$ patients did not show an attentional bias for positive words which are related to OCD, there was no evidence for the concern-relatedness hypothesis. Despite the similar set-up of the experiment, these results are in striking contrast with the Mathews and Klug (1993) data. These contradicting findings might be caused by differences between $O C$ patients and patients with other anxiety disorders. It is often remarked that OCD is strongly rellated with depression. Van Oppen and Arntz (1993) suggested that OC patients have characteristics of both anxiety and depressive disorders: with anxiety patients they share their concern about future catastrophic events and with depressive patients their perception of personal responsibility for this catastrophic event. But this does not appear to be a logical reason for $O C$ patients to be less emotionally involved in positive concern-related information than other anxiety patients. On the contrary, one would expect that OC patients are more involved as a result of this personal responsibility. There could be another reason for the contradicting findings; the statistical analyses of Mathews and Klug (1993) are not entirely adequate for testing the concern-relatedness hypow thesis, resulting in interpretation difficulties. Mathews and Klug carried out an ANCOVA with repeated measures, with colour naming times for neutral words as covariate. Consistent with our findings, they found a significant Group by Wordtype interaction, but they failed to clarify this interaction effect with pairwise comparilsons. Inspection of their data (table 3) revealed that, when the colour naming times of emotional words are adjusted for colour naming times of neutral words, the anxious subjects were relatively slowed down in colour naming anxiety-related words but accelerated in colour naming anxiety-unrelated words. Since no standard deviations are provided, it remains unclear whether the significant interaction effect is due to anxious subjects performing worse than normal controls on anxiety-related words or performing better than normal controls on anxiety-unrelated words (or a combination of two nonsignificant trends). If this interaction effect is not due to a deteriorated performance of anxious subjects on threat related stimuli, there would be no evidence for the claim, that anxious subjects selectively process information which is semantically related with their disorder.

Concerning the emotionality hypothesis our results are indefinite. There was no significant difference between the anxious and control group, but there was remarkable trend for anxious subjects to show relatively slower responses to disorderunrelated emotional stimuli. Except from the Martin et al. (1991) study, there are several other studies claiming emotionality effects. Although none of these studies can differentiate precisely between the emotionality or concern-relatedness hypothesis, since they did mot distinct between concern-related and concern-unrelated positive cues, they may be worthwhile to review. Richards and Millwood (1989) reported that high anxious normals did not show an attentional bias for positive words, whereas Mogg and Marden (1990) did find evidence for such attentional bias in a comparable study. McNally, Riemann, Louro, Lukach and Kimn (1992) found that panic patients responded more slowly to positive than to neutral cues and 
interpreted this finding as being consistent with the emotionality hypothesis. The data are difficult to expound however, since in contrast to the emotional stimuli, the neutral stimuli were nonlexical cues. From early Stroop experiments (Klein, 1964; La Heif, 1988) it is well-known that meaningless stimull cause less interference with colour naming. In the McNally et al. (1992) study, normal controls indeed also responded more slowly to positive words than to the neutral nonlexical cues. For the emotionality hypothesis, the critical test would compare anxious and normal subjects on their selective interference for positive words. However, this was not reported in the McNally et al. (1992) study. The differences between the two groups were nonsignificant for both the nonlexical neutral cues and the positive words, suggesting that the anxious subjects did not show an attentional bias for positive cues. Moreover, emotionality ratings did not correspond with the colour naming latencies. In the Mathews and Klug (1993) and in the present study it is found allso that word sets with similar emotionality ratings do not necessarily cause similar attentional biases. Thus evidence for the emotionality hypothesis is weak and inconsistent.

Taken together, reliable evidence is only found for the threat-relatedness hypothesils. Our data are inconclusive about the emotionality hypothesis of Martin et al. (1991), and failed to support the concern-relatedness hypothesis of Mathews and Klug (1993). With respect to the latter hypothesis, two possibilities are open. First, in contrast to other anxiety disorders, $O C$ patients may not be hyperattentive to positive concern-related cues. It is tempting to speculate what specific features of OCD may then be involved. However, this may be premature as the robustness of the concern-related attentional bias in other anxiety disorders still await consolidation. A third and conclusive experiment with a similar set-up is warranted. 


\title{
5.3 Attentional bias for appetitive cues: Effects of fasting in normal subjects ${ }^{4}$
}

\begin{abstract}
Summary
A long series of earlier studies demonstrated that fearful subjects selectively allocate attention to sources of perceived threat. Such attentional bias may not be limited to negative cues. It is hypothesized that attentional bias serves to enhance early identification of action-relevant cues and that such bias also occurs when subjects are confronted with positive valenced cues that evoke an urge to act immediately. In order to determine whether or not the attentional bias effect is limited to unpleasant stimuli, we studied the effects of 24 hours of fasting, focusing our attention on a possible attentional bias for (positively evaluated) food stimuli. The experiment controlled for emotionality effects.

It is shown that fasting results in a positive evaluation of food stimuli and an increased desire for these stimuli. The attentional bias effects found were slight and more likely attributable to the increased urge to act than to general emotionality effects. The power of the experimental manipulation and the computerized Stroop task are discussed.
\end{abstract}

\section{Introduction}

Attentional bias is one of the major topics in current cognitive approaches to emotional disorders. Several attentional bias tests have been carried out with various types of anxiety patients (for a review see Williams, Watts, MacLeod, \& Mathews, 1988). In almost every study published a disorder-related attentional bias is demonstrated in anxiety patients, i.e., spider phobics having an attentional bias for spider cues, obssesive patients for cues related to the obsession, etc. Evidence of attentional biases in depression is inconsistent. Gotlib and McCann (1984) found that depressive patients have an attentional bias for gloomy words, but MacLeod, Mathews and Tata (1986) argued that the researchers failed to assess anxiety in the depressed subjects. In a recent experiment, Mogg, Mathews, May, Grove, Eysenck and Weinman (1991b) showed that an attentional bias in depressed patients is more associated with anxiety than with depression.

The outcomes of these studies have led many to believe that attentional bias is specific for anxiety. A hypothesis frequently cited is that anxiety leads to increased attention to threatening events, which in turn maintains the anxiety (e.g. Mathews 
2. Eysenck, 1987\%. Hyperattention to anxiety-relevant cues could be functionally significant in that it optimally prepares anxious subjects to avoid or escape the feared object (Mackeod \& Mathews, 1991; MacLeod et al, 1986; Mathews, 1989). Attentional bias may enhance the early identification of anxiety-relevant cues and facilitate scape from potential danger (Lavy ${ }_{n}$ van den Hout, \& Arntz, 1993e).

The hypothesis that attentional bias is specific for anxiety disorders is refuted by the results of other experiments in which an attentional bias is demonstrated in patients with anorexia nervosa (Channon. Hemsley, \& De Sillva, 1988) and bulimia nervosa (Schotte; McNally, \& Turner, 1990), as well as in patients who attempted to commit suicide (Williams \& Broadbent, 1986). Normal subjects born in Northern Ireland do not show an attentional bias for violence-related words, while English subjects living in Northern lireland do (Giles \& Cairns, 1989). It could be argued that for all these populations the stimuli used contain an anxiety component. However, even if this is true, this argument does not account for data from a couple of other experiments. It remains an enigma why normals are hyperattentive to meaningful cues such as their own names (Maray, 1959). In low-anxiety normals with a repressive coping style an attentional bias for general emotional words has been demonstrated (Dawkins \& Furnham, 1989), as has such a bias for gloomy words in normal nonanxious subjects with hypomanic traits (Bentall \& Thompson, 1990). Martin, Williams and Clark (1991) argued that attentional bias in anxiety patients is not specific for threatening stimuli, because patients with generalized anxiety disorder also show an attentional bias for positive emotional words. These findings cannot be explained by the "anxiety-avoidance" hypothesis.

General emotion theorists, like Frijda (1986), suggest that emotions -negative and positive emotions alike reflect an action tendency. In the line with this postulation we hypothesized that subjects show an attentional bias for all stimull that evoke an urge to act immediately, i.e., not only an urge to avoid but also an urge to approach. When threatened, immediate avoidancelescape is obviously of vital interest. In the case of appetitive cues, approach can usually be postponed without threatening the integrity of the subject. Attentional bias for appetitive cues is only expected when immediate approach is of vital interest.

In normal subjects mainly a lack of the basic necessities of life le.g. drink and food) demands immediate approach behaviour. Channon and Hayward (1990), testing a different hypothesis, conducted an experiment in which normal subjects were instructed to fast for 24 hours. Compared to nonfasting controls, the fasting subjects were slower in their responses on a Stroop task with food stimuli. However, the emotional valences of the stimuli were not investigated. Thus critics can still claim that, as a result of the fasting, food stimuli arouse anxiety lor at least a negative evaluation) since the subjects are frustrated in partaking of those stimuli. Moreover, the Channon and Hayward study contained some methodological shortcomings. While all experimental Stroop words were thematically related to food, the neutral words were semantically unrelated. Mogg, Mathews, Eysenck and May (1991a) showed that subjects were generally faster to respond to categorized words than to uncategorized words in a lexical decision task. It is unclear how thematic relatedness between Stroop words affects colour naming latencies. Kaspi and McNally (1991) showed that blocked word presentation results in more interference than random word presentation. This suggests that the blocked format involves not only an attentional bias effect as a result of the presentation of a single item, but also a postattentional summation or rumination effect (McNally, personal 
communication). Card Stroop tests, as used by Channon and Hayward, seem to be even more susceptible to interstimulus rumination than blocked presentation of single words. Closer association between the food stimuli could have increased the rumination and with it the delay in collour naming. What is more, subjects were tested just once, leaving it unclear as to whether there were initial between-giroup differences. Finally, Channon and Hayward did not report how the time taken to colour name the words was recorded.

Even if the food stimuli did not arouse negative emotions and the above-mentio. ned flaws were of no importance, the set-up of the Channon and Hayward experiment precludes conclusions about our hypothesis that attentional bias is only shown for stimuli that evoke an urge to act. In order to show that it is this urge to act, rather than the emotionality of the words, that results in an attentional bias; it is necessary to add control words with comparable emotional valence for fasting subjects but which do not prompt to act. In order to confirm the hypothesis, a selective interference for food words rather than for positive control words has to be shown in fasting subjects.

In the present experiment, we repeated the fasting experiment using a computerized Stroop task. In order to secure pre-experimental similarity of the two groups, subjects carried out a conflict colour Stroop test before group assignment. Pilot studies have shown that correlations of response latencies of various Stroop tasks are 0.90 or higher, suggesting that performance on a conflict colour Stroop is an adequate variable for group assignment. In the experimental Stroop test, subjects were presented with food words, positive control words and neutral words. Care was taken to ensure that within each category all words were associated.

Attentional bias is usually not associated with enhanced memory for those stimuli. In most attentional bias studies, no memory bias has been found le.g. Ehlers, Margraf, Davies, \& Roth, 1988; Mathews \& MacLeod, 1986; Moggr Mathews, \& Weinman, 1989) and there is some experimental evidence that phobics show a negative memory bias for fear-relevant information (Landau, 1980; Watts, Trezise, \& Sharrock, 1986c; Mogg. Mathews, \& Weinman, 1987). The lack of memory bias could be the result of avoidance of fear-provoking stimuli (Mogg et al., 1987) or avoidance of full processing of phobia-related material [Watts \& Dalgleish, 1991). Since food stimuli are not fear provoking but appetitive cues, one would expect them to be processed without restrictions. Assuming that fasting subjects allocate relatively much attention to food stimuli, one would expect enhanced processing to result in a memory bias for food stimuli in those subjects. In the Channon and Hayward study, only a recognition test was performed and no bias was found. In the present experiment, memory was investigated with both a free recall and a recognition test.

\section{Method}

\section{Subjects}

Subjects were 40 healthy students - 20 males and 20 females - with no history of eating disorders. In order to check whether they were concerned about eating or dieting, they filled in the Restraint Scale (Polivy, Herman, \& Warsh, 1978) and the Dutch Eating Behaviour Questionnaire (Van Strien, Frijters, Bergers, \& Defares, 1986) before participating in the experiment. Subjects had to score within normal 
ranges (RS-score $<14$. DEBO sub-scales none higher than "above average"). Nine of the subjects (7) females and 2 males) failed to meet these criteria and were replaced. The mean age of the participating subjects was 21.6 years (range $19-28$ years). Subjects were paid for their participation and informed about the experimental procedure but not about the hypotheses being tested.

\section{Measurements}

Stroop test. Stroop words were presented to the subject via an Olivetti M250 microcomputer. The subject held a microphorie, which was connected to a voice level detector, in his/her hands. The computer clock stopped at the initiation of the subject's vocal response. The computer recorded the response latencies in milliseconds. Errors were marked via an interface button, operated by the experimenter.

Words appeared in the centre of the computer screen, preceded by a little fixation cross 2000 msec before word onset. Word sequence was determined randomly, but the sequence was identical for all subjects. Words were displayed in $8-\mathrm{mm}$ red, blue, yellow or green block letters. Subjects were asked to name the collours of the words as quickly as possible and to ignore the word content. The word remained on the screen until the subject reacted, with a minimum of $1000 \mathrm{msec}$.

Two Stroop tests were constructed: a conflict colour test and an experimental Stroop test. The conflict colour test consisted of 60 trials. The words presented were: red, green, yellow and blue. In the experimental Stroop test, three types of stimulus words were presented: 10 food words, 10 non-urgent positive words (related to holiday), and 10 neutral words (related to tools). The emotional valences of the words were tested in a few pilot studies. Care was taken to ensure that words were matched in terms of length and number of syllables in the Dutch language. Since familiarity to the stimulus words has proven to be of little interest in attentional bias studies (e.g., Foa \& McNally, 1986; McNally, Riemann, \& Kim, 1990b; Mogg \& Marden, 1990), the stimulus words were not matched in terms of frequency of usage. Table 5.3.1 shows the stimulus words used, translated into English. Each word was presented five times, making a 150-trials Stroop task. In modified Stroop tests, the response latencies within each subject show considerable interstimulus variability that may obscure significant group differences. In order to minimize the influence of irrelevant interstimulus variance, a large number of trials is needed. But even the presentation of 150 trials lasted only about ten minutes.

\begin{tabular}{lll}
\hline Food words & Holiday words & Tool words \\
\hline sandwich & fun & hemmer \\
pizza & sea & nail \\
cheese & beach & drill \\
soup & free & saw \\
omelet & terrace & axe \\
chinese noodles & voyage & screw \\
lasagnal & holiday & measuring-staff \\
chips & hotel & chisel \\
croquette & suitcase & shovel \\
cake & sun & tongs \\
\hline
\end{tabular}

Table 5.3.1: Stimulus words 
Recall and recognition tasks. For the free recall test, subjects were asked to write down ewery word they could remember from the experimental test. For the recognition test, the 30 words used in the experimental Stroop were typed on a sheet of paper, randomly alternated with 30 distractor words: 10 related to food, 10 related to holiday and 10 related to tools. Subjects were asked to indicate whether any of the words were presented in the experimental Stroop (forced choice task).

Emotional valences ( $E V$ ) and urge to act (UtA) scales. Both the emotional valen. ces and the urge to act were rated on $100-\mathrm{mm}$ Visual Analogue Scales (VASs). Emotional valence was measured because from the Channon and Hayward (1990) study it remained unclear how food stimuli are valenced by fasting subjects. On the EV scales, subjects rated the emotionality of the stimulus words in a nonverbal way: two Self-Assessment Manikins (SAMs) at the extremes of the VAS anchored the very negative (a miserable facial expression: EV score =0) and positive (a happy facial expression: $E V$ score $=100$ ), emotions between which the rating could range. On the UtA scale, the subjects rated how urgent it was to obtain a particular stimulus within an hour (ranging from 0 "acquisition can be delayed if necessary" to 100 "acquisition can impossible be delayed").

After both the conflict colour Stroop test and the experimental Stroop test, the subjects filled in the EV and UtA scales. The first time the EV and UtA scales were filled in, the stimulus words were randomly interspersed with distractor stimuli, in order to mask the words that were to be used in the experimental Stroop test.

\section{Procedure}

Subjects were tested twice. During the first session, they were presented with the conflict colour Stroop and the EV and UtA scales. Without the subjects' know. ledge, their mean response latencies on the conflict colour task were calculated and the subjects were assigned to either the fasting or nonfasting condition. A running average of response latencies was kept during group assignment to ensure that the groups did not differ in mean response latencies prior to the experiment. Moreover, care was taken to ensure that in both groups 10 male and 10 female subjects participated.

At the end of the first session, subjects were instructed either to eat normally (nonfasting condition) or to fast for 24 hours (fasting condition) before the second test, which took place exactly one week liater. Although subjects in the fasting condition were not allowed to eat anything for 24 hours, they were permitted to drink coffee, water, tea and "diet" soft drinks. They signed a consent that they would adhere to the fasting instructions and were made to believe that their urine would be examined in order to check whether they indeed had followed the fasting instructions.

The second session started with the experimental Stroop test. Immediately afterwards, memory was tested with first, the recall task, and second, the recognition task. Finally, subjects filled in the EV and UtA scales.

\section{Results}

The hypotheses tested in the presented study were the following:

1. Fasting subjects rate food words more positively than nonfasting subjects.

2. Fasting subjects show a greater urge to obtain food than nonfasting subjects. 
3. Fasting subjects show an attentional bias for food words, in contrast with both holiday and tool words.

4. When fasting subjects show an attentional bias for food words, they will also show an associated memory bias for those words.

The hypotheses were tested with ANOVAs with repeated measures. Subsequent unvariate tests were carried out, allowing for one-tailed testing of the hypothesized direction of the effects.

\section{Check for pre-experimental differences}

During the first session there were no differences between the prospective groups in EV ratings or in UtA ratings of food, holiday, and tool stimuli, nor were there differences in mean age or average response time $(P>0.500$ in all cases). Therefore, the groups could be considered as comparable before the experimental manipulation.

\section{The rating scales}

As can be seen in table 5.3.2, the three word types differed in emotional valence. Fasting subjects rated food words as positively as holiday words, while the nonfasting subjects rated food words less positively. In both groups holiday words were rated more positively than tooll words.

\begin{tabular}{lcccccc}
\hline & \multicolumn{3}{c}{ EV } & & \multicolumn{3}{c}{ UtA } \\
& Food & Holliday & Tool & Food & Holiday & Tool \\
\hline Fasting & 70.25 & 72.73 & 30.65 & 37.11 & 15.31 & 4.81 \\
& $(20.48)$ & $(13.81)$ & $(22.67)$ & $(27.14)$ & $(11.74)$ & $(6.79)$ \\
& & & & & & \\
Non- & 46.52 & 72.59 & 26.79 & 9.33 & 11.64 & 3.07 \\
fasting & $(21.20)$ & $(19.94)$ & $(18.85)$ & $(12.05)$ & $(11.01)$ & $(3.90)$ \\
\hline
\end{tabular}

Table 5.3.2: Means and standard deviations of raring on the Emotional Valence (EV) and Urge to Act (Ut,A) scales

When the ratings on the EV scales were submitted to a 2 (Condition) $x 3$ (Word type) ANOVA, the Condition $[F(1,38)=5.01, P=0.031]$ and Word type $[F(2,76)=$ $61.67, P<0.001 \rrbracket$ main effects proved to be significant, as was the crucial Condition $x$ Word type interaction $[F(2,76)=4.94, P=0.010]$. The fasting group rated food words more positively than the nonfasting group $\llbracket F(1,38)=12.97, P<0.001]$, but the groups did not differ in ratings of the holiday words $[\mathbb{F}(1,38)=0.001$, n.s. $]$ or tool words $[F(1,38)=0.342, n . s$.$] . In addition, the fasting group rated food words$ as positively as the holidlay words $[F(1,19)=0.20, n . s$.$] , while the nonfasting group$ rated the holiday words more positively than the food words $[F(1,19)=24.00$, $P<0.0011$.

In table 5.3 .2 it can be seen that for fasting subjects the need to obtain food stimuli was more urgent than that to obtain the other stimuli. Nonfasting subjects did not think it was really urgent to obtain any of the stimuli. Because the urge to 
act data were skewed, data were submitted to a logarithmic transformation prior to analysis. $A$ 2(Condition) $\times 3$ (Word type) ANOVA was then carried out on the UAA scales, showing a significant main effect of Condition $[F(1,38)=10.52, P=0.002]$ and Word type $[F(2,76)=38.91, P<0.001]$ and the predicted interaction effect of Condition $\times$ Word type $[F(2,76)=9.73, P<0.0011$. As expected, the fasting group thought it more urgent than the nonfasting group to get the food stimuli within an hour $[F(1,38]=21.80, P<0.001]$, again, the groups did not differ in their urge to acquire holiday stimuli $[F(1,38)=2.27, n$.s.] or tool stimuli $[F(1,38)=1.78, \pi$. s. $]$. Moreover, the fasting group found it significantly more urgent to obtain food stimuli than holiday stimuli $[F(1,19)=35.58, P<0.001]$, while the nonfasting group did not. In fact, there was a marginal trend for nonfasting controls to rate holiday words more urgent than food words $[F(1,19)=3.07, P=0.095]$.

\section{The attentional bias}

Before analyzing the response latencies, trials with errors were excluded. Errors were extremely rare; they were either marked by the experimenter or indicated by response latencies less than $300 \mathrm{msec}$ or more than $3000 \mathrm{msec}$. The response latencies for the three word types were averaged for each subject and are displayed in figure 5.3.1.

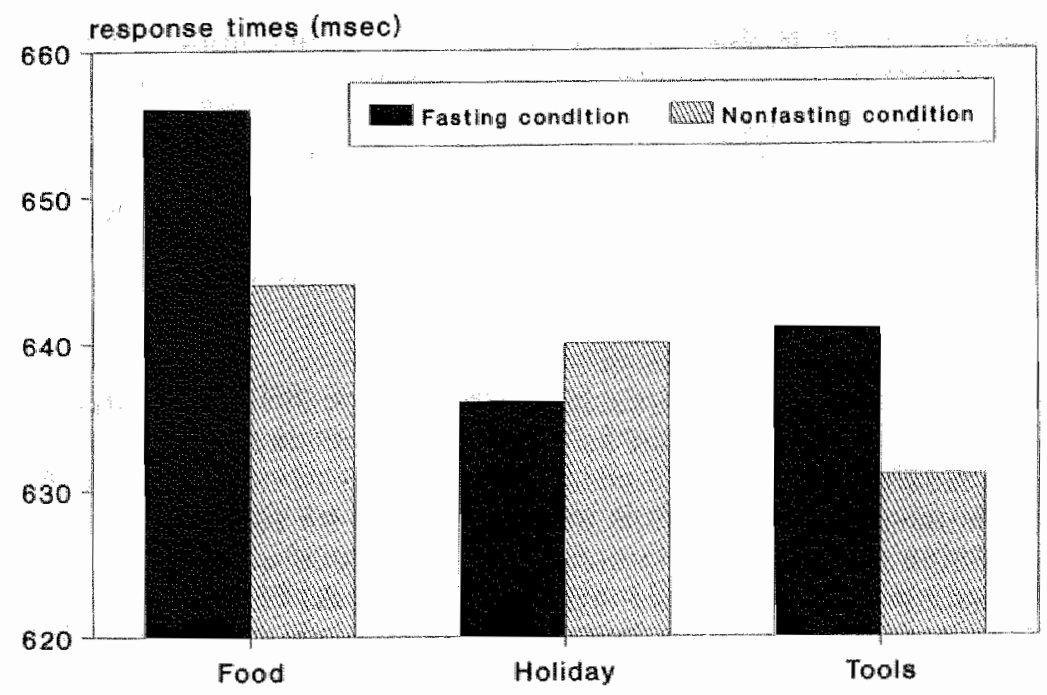

Figure 5.3.1: Response latencies imsecl for the three word types

Overall, response latencies tended to be longer in the case of food words. This delay was more pronounced in fasting subjects than in nonfasting subjects. A 2(Condition) $\times 3$ (Word type) ANOVA showed both a significant main effect of Word type $[F(2,76)=7.65, P=0.001]$ and a marginal Condition $x$ Word type interaction 
$[F(2,76)=2.43, P=0.095]$. There was no main effect of Condition $[F(1,38)=0.6$, n.s.J, i.e. in general the fasting subjects did not respond slower than the nonfasting controls.

Usually, selective interference is determined by calculating a difference score between the latencies for experimental and control words. Because we used two types of control words (neutral and positive), two interference scores were calculated: food versus tools interference and food versus holiday interference. Univariate analyses of the two interference scores failed to demonstrate a group difference in food versus tools interference $[F[1,38]=0.03$, n.s.] but did show a food versus holiday interference $[F(1,38)=3.32, P=0.038$, indicating that the difference between food and holiday stimuli was larger in the fasting condition than in the nonfasting condition.

\section{The memory bias}

Because errors were rare in the free recall test, only the correct answers were analyzed. A $2 \times 3$ ANOVA exposed no significant main or interaction effects $(P>0.100$ in all cases).

Recognition memory was exämined using signal detection analysis (McNicol, $1972)$. Sensitivity $\left(\delta^{\prime}\right)$ scores of the three word types were submitted to a $2 \times 3$ ANOVA with repeated measures. There were no group differences in main or interaction effects $(P>0.100$ in all cases); thus, there was no evidence of a memory bias for food words in the fasting subjects. Response biases revealed a strong tendency in both groups to answer "no" to all wordls presented (mean $\beta=44.9$ ). Analysis of the response biases $(\log \beta)$ showed only a main effect for Condition $[F(1,38)=7.45, P=0.010$ l, reflecting a tendency in fasting subjects to answer less in the negative than the nonfasting subjects. However, there was no Condition $x$ Word type interaction effect and, therefore, no evidence of a memory bias for food words in fasting subjects.

\section{Discussion}

The present study revealed that fasting resulted in a more positive evaluation of food stimuli. Fasting subjects evaluated food as positively as holiday. Thus there is no indication that the fasting instructions caused food stimuli to be estimated as unpleasant or tension-inducing and a potential attentional bias for food cues could, therefore, not be attributed to the anxiety-arousing effect of food stimuli. Twentyfour hours of fasting resulted in an increased urge to obtain food. The experimental manipulation therefore appeared adequate to test the hypothesis that attentional bias is not only shown for anxiety-related cues but for all stimuli that evoke an urge to act immediately.

Evidence of an attentional bias for food stimuli in fasting subjects is inconsistent, depending on the control stimuli used. Conform the third hypothesis, fasting subjects, compared with nonfasting controls, showed more colour naming interference for food words than for holiday words. However, when tools words are used as control stimuli, no attentional bias was found. This unexpected finding is difficult to interpret, since there is no logical reason for fasting subjects to respond slower to tools stimuli than nonfasting subjects. One might suggest that fasting subjects may be less concentrated in general, but the nonsignificant main effect of condition 
counters this argument. Fasting subjects rated food stimuli as positive as holiday stimuli, but only deteriorated in colour naming the food stimuli. Although the results obtained with the Stroop test are inconsistent, it is thus obvious that the attentional effects are not due to emotionality effects.

Undoubtedly, the attentional bias effects found in the present experiment are smaller than those in the Channon and Hayward (1990) study. This may be attributed to the format in which the Stroop stimuli are presented. With a card Stroop, as used by Channon and Hayward, the attentional bias effect can be inflated by the effects of rumination, while with a computerized random presentation of single stimuli, possibilities for further processing are minimized. Moreover, it could be that although the manipulation produces an increased desire for food, it is not strong enough to result in an unambiguous attentional bias in normal subjects. The mean rating of fasting subjects on the UtA scale indicates that, although they found it more urgent than nonfasting subjects to obtain food, there was no uncontrollable urge to act immediately, as there sometimes is in phobic patients confronted with a feared stimulus. Most likely, the combination of Stroop format and strength of manipulation resulted in fragile effects.

In concurrence with previous research, no memory bias for food stimuli was found in fasting subjects. Without an unambiguous attentional bias, this finding cannot be interpreted as refuting the hypothesis. Even when relevant information is fully processed, in normal subjects no memory bias can be expected without a selective attention to food stimuli.

In sum, the attentional bias effect does not seem limited to either anxiety patients or threatening stimuli. In normals, an attentional bias can be induced for appetitive cues; although the effects are not very strong. As far as an attentional bias for positive cues is shown, it is more likely due to an urge to act immediately than to an effect of emotional valence.

In general, attentional bias data obtained from normal, nonanxious subjects lack consistency. When nonanxious subjects are presented with neutral and emotional/ threatening stimuli, an attentional bias is sometimes found for the latter (Cook \& Johnston, 1989; McKenna, 1986; Williams \& Broadbent, 1986) and sometimes no attentional bias is found at all (Martin et al. . 1991; Mathews \& MacLeod, 1986; Mogg et al. , 1989). In other studies, nonanxious subjects show a facilitated response for threat cues (e.g. Macleod \& Mathews, 1988; MacLeod et al, 1986). One possible reason was mentioned before, namely that in normal subjects it is difficult to evoke an urge to act immediately that is quantitatively comparable to the avoidance urge of anxious subjects. In a recent study. MacLeod and Hagan (1992) offered an intriguing alternative explanation: both clinical anxious subjects and normals with high trait anxiety selectively process threat-related information at an automatic level of cognitive functioning. However, normal subjects, unlike anxious patients, are capable of negating this automatic bias by means of consciously mediated strategies, thereby limiting the disturbing effects of the threatening information. MacLeod and Hagan (1992) presented normal subjects with neutral and threatening stimuli under masked and unmasked exposure conditions. In the masked exposure condition, each word was presented for $20 \mathrm{msec}$, after which it was replaced by a mask. Under these conditions conscious awareness of the stimulus word is prevented, but semantic processing is not. Consistent with their hypothesis, they found that in normal subjects, trait anxiety was associated with attentional 
bias for threatening words only when the stimuli were presented under masked exposure conditions.

If normal subjects indeed tend to actively counteract automatic tendencies to attend to threat cues, they may do the same in the case of appetitive cues that they know cannot be obtained immediately. It would be interesting to repeat the present experiment with masked word presentation in order to see whether fasting normal subjects show a more prominent attentional bias when only the automatic level is measured and when possibilities for coping strategies are minimized. 


\subsection{Thought suppression induces intrusions ${ }^{5}$}

\section{Summary}

An experiment was conducted to investigate whether deliberate attempts to avoid a thought content have paradoxical effects. Twenty-four subjects received thought suppression instructions, 16 neutral instructions. Thought suppression resulted in an increased number of occurrences of the thought to be suppressed. The contribution of the results to the theory of obsessions is discussed.

\section{Introduction}

How abnormal is it to have obsessions? Interested in finding the answer to this question, Rachman and De Silva (1978) investigated whether there is a "normal" variant of the clinical obsession. Surprisingly, approximately $80 \%$ of their normal subjects occasionally experienced intrusions that resembled the form and content of abnormal obsessions. Salkovskis and Harrison (1984) replicated this study and found a comparable percentage of intrusive thoughts in normal subjects. Thus, intrusions seem to be frequent experiences and, because of the many similarities between them, it seems likely that "pathological" obsessions and "normal" intrusions form part of one and the same continuum (Salkovskis, 1985).

The next question to be asked is: in what respects can intrusions and obsessions be distinguished from one another? The Rachman and De Silva study found differences in the following parameters: intensity and frequency of occurrence, and effects of the unwanted thought, such as discomfort, resistance and dismissability. Another potentially important difference in the effects is that people with obsessions feel a greater need to avoid or neutralize the unwanted thought (Salkovskis, 1985).

Several authors have wondered whether there is a possible relationship between the frequency and intensity of unwanted thoughts, on the one hand, and the consequences of such thoughts, on the other. Evidence for such a relationship is mixed. For instance, Parkinson and Rachman (1981) found a relationship between the frequency of an intrusion and its dismissability, while a replication study by Salkovskis and Harrison (1984) failed to find one. In this context it is interesting that Rachman and De Silva (1978) found that the frequency, duration, and discomfort of obsessions decrease with practice. These results could be explained theoretically with the help of the Rachman hypothesis (Rachman 1971, 1978), namely, that obsessions are noxious stimuli to which a person is not habituated because of avoidance and neutralizing activities. The model suggests that clinical obsessions are 
maintained because (cognitive) avoidance/neutralizing activities prevent extinction of experienced noxiousness. In "normal" intrusions experienced discomfort is extinguished by exposure and non-avoidance. Recent data suggest that cognitive avoidance may riot only serve to maintain obsessions. Attempts to avoid cognitively may also produce or lead to an increased frequency of the avoided thought.

Relevant experimental data were provided by the "white bear" experiment of Wegner, Schneider, Carter and White 11987). Normal subjects were asked to verbalize their stream of consciousness for two 5 -minute periods. One group was instructed to try not to think of a white bear during the first 5 -minute period and to press a bell-button when this thought did occur to them. During the second period, they were instructed to think of a white bear as much as possible (initial suppression group). The ather group received the instructions in reverse order (initial expression group). The thought suppression instructions had two effects. First, there was a paradoxical effect: subjects were unable to suppress their thoughts about a white bear when requested to do so. Second, there was a rebound effect: the initial suppression group was much more preoccupied with these thoughts during the expression phase than the initial expression group was.

In the present context, the apparent inability of subjects to suppress white bear thoughts is of interest. It suggests that efforts to suppress a thought are counterproductive and result in an increased likellihood of the thought coming to mind. Still, while the Wegner data are surely suggestive, they preclude definite conclusions. First, it is not possible to deduce the pure and direct effect of thought suppression from the Wegner data, because the control condition received explicit thought expression instructions which prevent a direct comparison of the number of intrusions in the suppression and the control condition. The conclusion that thought suppression induces intrusions could only be made if the thought suppression group was to experience more intrusions than a control group that had received no explicit thought expression but rather neutral instructions. Thus, although the Wegner study made it plausible that thought suppression has a paradoxical effect, it is still not clear whether attempted thought suppression is sufficient to cause intrusions. Second, it is possible that the presence of the bell-button had, in itself, become a reminder to the subjects of the white bear, thus increasing the number of intrusions in an artificial way. Wegner investigated the role of reminders (Wegner, 1989) and found that environmental stimuli can serve as cues to the to be suppressed thought. Cue (bell-button) dependent recall (white bear) may thus be an alternative explanation of the data.

Considering the potential relevance of the Wegner paradigm to understanding obsessions and other unwanted thoughts, we tried to replicate the Wegner study with a design as clear and straightforward as possible. We concentrated on the paradoxical effect of thought suppression. Attention was paid to the above mentioned interpretational problems created by the original design. Besides, we wondered if the thought suppression effects were not due to stimulus qualities; a white bear is obviously a very striking stimulus, and one might argue that a less striking subject would be easier to suppress. In the present study, a non-extravagant thought content was used as to be suppressed notion. Furthermore, we built in a manipulation check to see to what degree subjects have actually tried to suppress the thought. 


\section{Method}

\section{Subjects}

Forty healthy students - 20 males and 20 females - volunteered to participate in the experiment. Their mean age was 20.7 years (range $18-29$ years). They were paid for their participation and informed about the experimental procedure. Subjects were randomly assigned to one of two conditions: a thought suppression group ( 24 subjects) or a control group (16 subjects).

\section{Procedure}

Subjects were told that the study focused on cognitive processes and that they would be asked to spend two 5 -minute periods alone in a soundproof room, continuously reporting about everything that came to their minds. They were informed about the tape recorder and assured that their identities would remain anonymous.

The experimenter made sure that each subject understood the instructions and then left the room. The subject closed his/her eyes and started to think aloud. The purpose of this first period was to practice verbalizing the stream of consciousness. After 5 minutes, the experimenter returned and asked if the subject had experienced any difficulties in executing the task; some subjects report that they had at the beginning, but at the end of the practice-period no one had difficulties anymore to verbalize the stream of consciousness. Then the thought suppression group was given the instructions to verbalize their thoughts as they had done before, but with one exception: they were to try not to think of "vehicles", just like the Wegner et al. subjects were asked not to think of a white bear. The results of pilot studies showed that it was necessary to add something else to the instructions, namely, that the subjects should not use deliberate distraction strategies. The control group was also told to verbalize their thoughts as before. They were also told that they were free to think whatever they wanted. "You can, for instance, think of vehicles, but you can also think of the animals living in the zoo, or anything else that happens to come to your mind". Both groups were encouraged not to concentrate on one specific thought, but merely to follow their stream of consciousness. Care was taken that the cues "vehicles", "animals living in the zoo" and "anything else that happens to come to your mind" wwere mentioned only two times in each of the two types of instructions. Then the experimenter left the room again and the subject verbalized the stream of consciousness for a second time. After 5 minutes the experimenter came back and gave the subject a questionnaire (manipulation check).

\section{Measurements}

The subjects' verbalizations of the stream of consciousness were tape recorded and analyzed for the number of intrusions, i.e. the number of occurrences of the thought that was to be suppressed. Counted were both occurrences of the word "vehicles" and examples of the concept of "vehicles" such as car, train or bicycle. After the experiment all subjects received a questionnaire on which they were asked to what degree (1) they had tried not to think the suppressed thought and (2) they felt they could verbalize their thoughts. In order to obtain some additional information, the thought suppression group was also asked to what degree (3) they felt they had succeeded in suppressing the thought and (4) they had tried to use distracters. The questions could be answered on four-point scales. 


\section{Results}

In order to control for differences in verbalization ability, a Mann-Whitney test was performed on the second part of the questionnaire. Surprisingly, although both groups had practiced before, there was a nonsignificant trend for the suppression group to verbalize their thoughts better than the control group $\mathbb{U}=85.0$. $W=279.0, P=0.070$. Then the hypothesis that thought suppression instructions induce a preoccupation with the suppressed thought was tested. When the tapes were analyzed, it appeared that the average number of vehicles mentioned was 3.63 (s.d. 3.70) for the thought suppression group and 0.69 (s.d. 0.79 ) for the control group, as shown in figure 5.4.1. The difference between the two groups was strongly significant $(t(38)=3.76, p<0.001)$

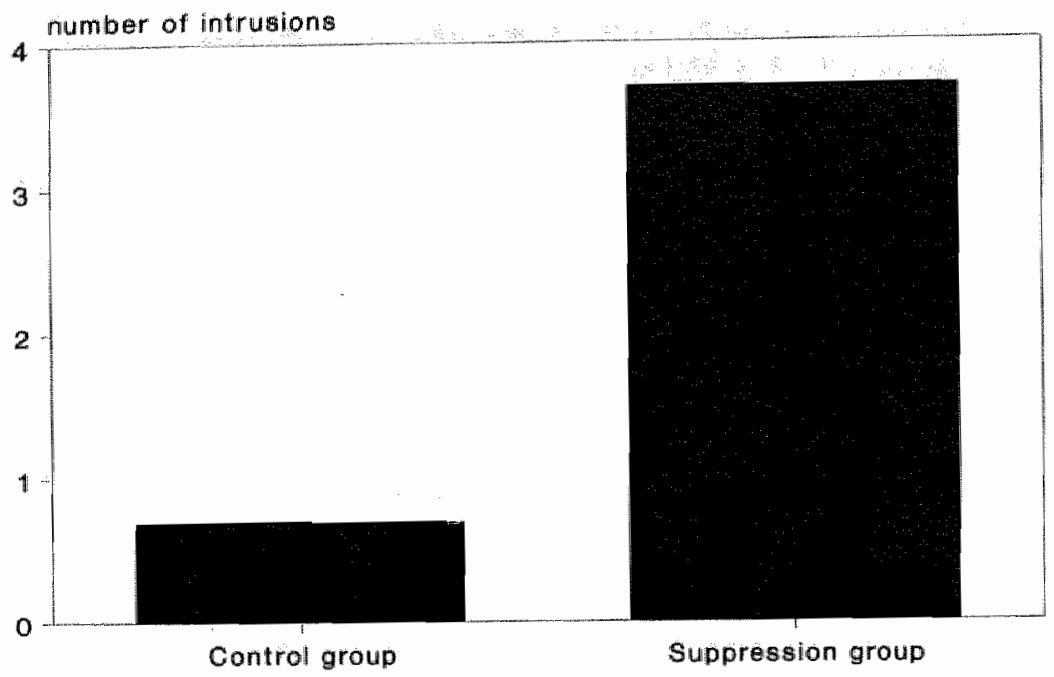

Figure 5.4.1: Number of intrusions during a 5iminute period of verbalizing the stream of consciousness fcantrol groupl or verbalizing with thought suppression (suppression group)

It could be argued that the number of intrusions mentioned, correlate with the verbalization ability and that the difference in the number of intrusions is an artefact from the difference in verbalization ability between the two groups. In order to control for this possibility, a Pearson's correlation between the verbalization ability and the number of intrusions was calculated. The correlation does not reach significance $(r=0.24, P<0.09)$, thus no evidence was obtained for the proposed artefact.

In order to check whether the thought suppression group had actually tried to suppress their thoughts about vehicles, a Mann-Whitney test was performed on the first part of the questionnaire. The thought suppression group had suppressed the thought significantly more than the control group $(U=76.5, W=388.5, P=0.003)$. 
Moreover, subjects in the suppression group reported some doubt as to their success in suppressing the thought; their mean score on the third question was exactly between "I succeeded" and "I did not succeed". They also indicated that they had not tried to distract themselves by concentrating on one specific thought.

\section{Discussion}

The results of the earlier Wegner et al. study indicated that attempts to suppress a specific thought may have a paradoxical effect, but some design features made it hard to assess the relevance of the results to the understanding of obsessive compulsive disorder.

In this study, attention was specifically paid to two design characteristics that could allow for alternative explanations. The saliency of the stimulus (1) and the association between the suppressed thought and the presence of environmental stimuli (2) could both have caused intrusions to be artificially triggered. The role of the first factor was limited by using a non-extravagant thought content ("vehicles") and the role of the second factor by not using a bell-button and by asking the subjects to close their eyes when they verbalized their thoughts so that they could not see stimuli that were present in the room. The conclusion that the Wegner (1987) data are not an artifact of the bell-button procedure is corroborated by the psycho. physiological findings reported after completion of this study. Merckelbach, Muris, van den Hout and de Jong (1991) found that suppression maximalizes the number af spontaneous fluctuations in Skin Conductance Response, related to the to be suppressed thought. Likewise, Wegner, Short, Blake and Page (1990) reported that the suppression of an exciting thought resulted in an elavation of Skin Conductance Levell.

The most important improvement of the present study was that the design of the study allowed for a direct comparison of the thought suppression and control groups. The results show that the thought suppression group mentioned more thoughts about vehicles than the control group, despite the fact that the experimenter used the word "vehicles" an equal number of times. The simplicity of the design makes this result straightforward: thought suppression has a paradoxical effect and induces intrusions. Thus, the results of the present study confirm and give stronger support to the results of Wegner et al. (1987).

How does thought suppression produce its paradoxical effect? It may be argued that this effect is merely due to a greater saliency of the thought due to suppression instructions and not to the very process off suppression. Although there is no obvi.ous reason why an external suppression instruction should have different effects than a selfinstruction, this possibility cannot be excluded by means of the present design. It can be useful to carry out an experiment with a control instruction which result in increased saliency, but not in thought suppression.

Perhaps increased saliency plays an important role in another way: the process of suppression may result in an increased saliency of formerly neutral thought content, and intrusions may be the consequence of this increased saliency. Salkovskis (1985) proposed a cognitive model of obsessions in which he stated that all intrusive thoughts are triggered by internal or external stimuli and that "the reaction of the individual experiencing this "ntrusion will be determined by the extent to which its occurrence is salient for the person concerned". If the intrusion has a strong negati- 
ve emotional valence, this may increase the saliency of the thought and may produce an urge to neutralize and avoid the thought. Attempts to accomplish the latter will have a paradoxical effect and will, in the end, result in a vicious circle of more suppressing and more subsequent intrusions.

As mentioned earlier, subjects in this experiment were urged not to employ cognitive distraction strategies, and it is unclear if comparable results would have been obtained if distraction was allowed for. Deliberate efforts to distract oneself from unwanted thoughts (e.g. counting rituals) or to engage in neutralizing thinking are characteristic of many clinical obsessions. It would be worthwhile experimentalIy exploring the conditions under which distraction/neutralizing can or cannot block the paradoxical effects of thought suppression. It would also be interesting to investigate whether and how negative mood interacts with the observed phenomenon. Furthermore, one wonders if paradoxical thought suppression effects display a different pattern when idiosyncratic/personally relevant intrusions are used instead of affectively presumable indifferent cues like white bears or vehicles.

The idea of thought suppression having paradoxical effects is not new; in fact the first white bear anecdotes were attributed to Dostoyevski (Wegner et al., 1987) and Tolstoy (Wegner, 1989). Meanwhile, the thought suppression set-up provides a potentially fruitful paradigm for further controlled study of unwanted thoughts that may become clinical problems. 


\title{
5.5 Cognitive avoidance and attentional bias: Causal relationships ${ }^{6}$
}

\begin{abstract}
Experimental evidence indicates that anxious subjects show an attentional bias towards threatening information, but it has also been suggested that cognitive avoidance plays a role in anxiety. It was hypothesized that cognitive avoidance is causally involved in the emergence of attentional bias. An experiment was conducted with normal subjects to investigate whether a strong motivation for cognitive avoidance results in an attentional bias towards the (formerly neutral) subject to be avoided.

Forty-five subjects were instructed to suppress all thoughts about numbers, forty-five subjects received control instructions. Both groups carried out a modified Stroop test, including both number words and non-number control words. Compared with the control group, the thought suppression group showed an attentional bias towards number words, due to selective allocation of attention to number stimuli. Alternative interpretations, like priming and other unintentional effects of the experimental manipulation, are discussed but do not seem to be plausible. A functional relationship between motivation for cognitive avoidance and attentional bias is proposed.
\end{abstract}

\section{Introduction}

In the last decade, an increasing interest has emerged in the role of cognitive processes in the progress and maintenance of anxiety disorders. There are at least two lines of research: one line is to start from thought contents and the other from information processing. Clinical research concentrated on the former and, by making use of self-report techniques, it was found that anxiety patients reported having thoughts about personal danger at times of increased anxiety (Beck, Laude, \& Boh. nert, 1974; Hibbert, 1984).

Experimental research has mainly focused on a presumed disturbance at the input-side of information processing: anxiety patients show an attentional bias in favour of threatening information. Disorder-related attentional biases have been demonstrated in panic patients (McNally, Riemann, \& Kim, 1990b), spider phobics (Lavy, van den Hout, \& Arntz, 1993d; Watts, McKenna, Sharrock, \& Trezise, 1986a), social phobics (Hope, Rapee, Heimberg, \& Dombeck, 1990), patients with generalized anxiety disorder (Mathews \& MacLeod, 1985; Mogg, Mathews, \& Weinman, 1989), patients with obsessive compulsive disorder (Foa \& McNally, 1986) or

Lavy, E.H., \& Hout, M.A. van den (1993). Cognitive avoidance and attentional bias: Causal relationships. Cognitive Therapy and Research (in press). 
post-traumatic stress disorder (Cassidy, McNally, \& Zeitlin, 1992; McNally, Kaspi. Riemann, \& Zeitlin, 1990a). The conclusion that anxious subjects selectively allocate more attention to threatening environmental information seems to be justified.

How do we account for attentional bias? Anxious subjects orientate towards threat cues in the (pre)attentive phase, but this does not imply that thev also elaborate threatening information better than normals. On the contrary: there are some indications that they have a relatively poor memory for threat-provoking stimuli $(e, g$. Foa, McNally, \& Murdock, 1989a; Landau, 1980; Mogg, Mathews, \& Weinman, 1987; Watts, Sharrock, \& Trezise, 1986b; Watts, Trezise, \& Sharrock, 1986c). It has been suggested that the poor memory effects reported result from cognitive avoidance of threat-relevant material (Foa \& Kozak, 1986; Mathews \& MacLeod, 1987). The aim of cognitive avoidance would thus be to abort further processing of threatening material (Williams, Watts, MacLeod, \& Mathews, 1988).

There is some evidence that cognitive avoidance and attentional bias are related. Bentall and Thompson (1990) found that normal subjects with a hypomanic personality trait showed an attentional bias towards depressive, but not towards manic, words. Interpreting the data in a psychodynamic way, they found the results consistent with the hypothesis that hypomanic traits reflect a sustained defence against depressive feelings. Dawkins and Furnham (1989) tested normal subjects with a repressive coping style. Compared with both low-anxious and anxious normals the repressors showed an attentional bias towards emotional words. Dawkins and Furnham (1989, p.385) suggested that "these subjects suppress negative emotions because they find the notion of experiencing them more unpleasant and unacceptable (and thus more threatening than low and high anxious persons". However, it is difficult to understand why low-anxious repressors find emotional words even more threatening than anxious subjects. The results of these studies could be explained from a different viewpoint. Is it possible that cognitive avoidance is causally involved in the emergence of attentional bias? The hypothesis to be tested here is that a strong motivation for cognitive avoidance, independent of anxiety or other negative emotions, is sufficient to produce an attentional bias. To test this assumption in an experimental design, we decided to link two paradigms in one study. The attentional bias paradigm was a computerized Stroop task; the cognitive avoidance paradigm was a "thought suppression" task (Lavy \& van den Hout, 1990; Wegner, Schneider, Carter, \& White, 1987). In thought suppression task, subjects are instructed to suppress a certain thought content deliberately for a couple of minutes. This thought suppression instruction has the paradoxical effect that subjects become preaccupied with the thought to be suppressed (Lavy \& van den Hout, 1990; Wegner et al., 1987). The results of a manipulation check are important for the present study. These showed that the subjects, as a consequence of a thought suppression instruction, did indeed try to suppress the thought (Lavy \& van den Hout, 1990).

Assuming that the thought suppression paradigm provides a relevant model for cognitive avoidance, it was hypothesized that experimental thought suppression is sufficient for attentional bias to occur. In a former experiment (Lavy \& van den Hout, 1991) we found that the effects of a thought suppression instruction were fragile; the subsequent attentional bias was small and of short duration. Our main concern was that the Stroop task required so many cognitive resources that, as the experiment progressed, the thought suppression instructions were over-ridden. We therefore decided to carry out a new experiment with more stringent thought suppression instructions. In order to prolong the effect of the thought suppression 
instruction, we reminded the subjects of these instructions halfway through the Stroop test.

\section{Method}

\section{Subjects}

Ninety healthy students - 45 men and 45 women - volunteered to take part in the experiment. Their mean age was 21.0 years (range 18-29 years). They were paid for participation and were given information about the experimental procedure.

\section{Design}

Subjects formed part of either the Thought Suppression group (45 subjects) or the Control group (45 subjects). In order to achieve pre-experimental similarity between the groups, subjects carried out a short conflict colour Stroop test before being assigned to a group. A running average was kept during group assignment to ensure that the groups did not differ pre-experimentally in mean response latencies. Pilot studies have shown that correlations of response latencies off various Stroop tasks are 0.90 or higher, thus performance on a conflict colour Stroop seems to be an adequate variable for group assignment. Care was taken that men and women were allocated to the two groups equally. The two groups were thus similar with regard to mean pre-experimental latency scores, standard deviation of pre-experimental latency scores and number of men and women participating.

\section{Apparatus}

An Olivetti M250 microcomputer with a colour monitor presented the Stroop words to the subject and recorded the response latencies per word in milliseconds. A Sony Cardoid Dynamic microphone F560, which the subject held in his/her hands, was connected to a voice level detector which stopped the computer clock at the initiation of the subject's vocal response. Errors were marked by means of an interface button, operated by the experimenter.

\section{Characteristics of the Stroop test}

The words were presented in the centre of the computer screen. Words appeared in $8 \mathrm{~mm}$ block letters in one of the four possible colours: red, blue, yellow or green. The appearance of both words and colours was random, the only restriction being that, during the test, each word was presented five times and the same colour could not occur more than twice in succession. The word remained on the screen until the subject had reacted, but for no less than $1000 \mathrm{msec}$. and no more than $3000 \mathrm{msec}$. If the voice level detector had not recorded a response within 3000 msec, the trial was considered as missing and an error was registered.

\section{Procedure}

Subjects were tested individually in a dimly lit and sound-attenuated room. They were told that the focus of the study was the occurrence of types of brain waves during concentration tests and that the computerized Stroop task was a test for concentration. They were presented with the conflict colour task (24 trials). After this short test, without the subjects' knowing it, their mean reaction times were 
calculated and the subjects were assigned to one of the two groups: the Thought Suppression (TS) or the Contral (C) group.

All the subjects were then given basic (though erroneous!) information about the functions of the left and right hemisphere of the brain. They were told that the left hemisphere is involved in processing "verbal information" and the right hemisphere in processing "numbers". They were made to believe that we could record and locate relevant brain waves with two (dummy) electrodes applied to their temples. After fixing these electrodes, the subjects received subsequent information. The subjects in the TS group were instructed to suppress all thoughts about "numbers" during the next concentration test. It was suggested that we could monitor them: if they should think of numbers, our electrodes would record the brain waves due to activation of the right hemisphere. Subjects in the C-group were informed that we would measure brain waves during the next test, so that "we can see whether you think of numbers or think verbally". They were told there was nothing they would have to do, except concentrate on the test. In order to ensure that for both groups the cue "numbers" was mentioned only three times, and with similar emphasis, all instructions were tape-recorded.

Before the Stroop task presentation, the subjects in the TS-group were asked to "practise" thought suppression for two minutes and subjects in the C-group were asked to sit quietly and think of nothing in particular for two minutes.

The subjects in both groups were then presented a 100-trial Stroop test: 50 trials involving number words and 50 control words. Half-way through, after 50 trials, the test was interrupted. The subjects were toll that the experimenter wanted to check the brain activity data. The experimenter repeated the instructions, either that the subject had to suppress all thoughts about numbers or that we measured whether he/she thought about numbers or verbally. Care was taken that the "numbers" cue was mentioned only once to subjects in both groups. The Stroop test was then continued. After this test, the subjects filled in a manipulation check form isee below). Finally subjects were debriefed and paid.

\section{Stimulus words}

The number words used in the Stroop test were: TWO, THREE, FOUR, FIVE, SEVEN, EIGHT, TWELVE, THIRTEEN, FIFTY and HUNDRED. Control words were also taken from one specific category, because thematic (un)relatedness of stimuli can affect reaction times (Mogg, Mathews, Eysenck, \& Mav, 1991a). The control words were all substance words: WOOD, IRON, CONCRETE, PLASTIC, PAPER, STEEL, GLASS, COTTON, CARDBOARD and STUFF. These words were matched with the number words for number of syllables, total length and frequency in the Dutch language (Uit den Boogaart, 1975).

\section{Manipulation check: Visual Analogue Scales}

In order to see if the experimental manipulation was successful, all the subjects scored six $100 \mathrm{~mm}$ Visual Analogue Scales (VAS's) as to what degree: (1) they had tried not to think of "numbers" (2) they had tried not to think of other issues than "numbers" (3) they had tried to distract themselves from thinking of "numbers" (4) they had thought of "numbers" during the test (in percentage of time) (5) they were distracted from colour naming by the number words (6) they were distracted from colour naming by the substance words. 


\section{Results}

Data on one subject in the C-group was lost, due to computer failure. Analyses were therefore carried out for 89 subjects.

\section{Manipulation check}

The TS- and C-group VAS scores differed highly significantly on the first five VAS-scales, but not on the sixth one (see table 5.5.1). The subjects in the TS-group tried harder to suppress all thoughts about "numbers" and to distract themselves. from these thoughts. Despite their efforts, they spent more time thinking of "numbers" and were distracted more by the number words from colour naming than the C-group. Thus the experimental manipulation had succeeded. Interestly, the subjects in the TS-group took less pains to suppress other thoughts during the Stroop task than did those in the C-group.

\begin{tabular}{|c|c|c|c|}
\hline Manipulation checks & mean TS (s.d.) & mean C (s.d.) & $t(87)$ \\
\hline Suppression numbers & $82.84(14.21)$ & $23.90(20.48)$ & $15.80^{\cdots "}$ \\
\hline Suppression other thoughts & $22.82(28.96)$ & $35.90(25.80)$ & $-2.25^{\circ}$ \\
\hline Distraction & $81.84(20.39)$ & $29.07(26.55\}$ & $10.53^{\cdots}$ \\
\hline Percentage of time & $29.33(19.53)$ & $18.59(11.20)$ & $3.17^{\cdots}$ \\
\hline Distracted by number words & $48.53(29.22)$ & $28.93(22.63)$ & $3.53^{\cdots}$ \\
\hline Distracted by substance wards & $19.73(20.13)$ & $23.6\}\{18.90\}$ & .0 .94 \\
\hline
\end{tabular}

Table 5.5.1: The manipulation check: mean VAS scares (mm)

\section{Stroop response latencies}

Before analyzing the response latencies, trials with errors were excluded. Errors amounted to less than 2 percent of the trials and were either marked by the experimenter or indicated by response latencies which were less than $300 \mathrm{msec}$ or more than 3000 msec.

Trials were split into two blocks of fifty, allowing for time course investigation. The response latencies for number and substance words were averaged per block for each subject (see figure 5.5.1).

The mean response latencies for the first and second blocks of trials were analyzed via a 2 (Group: TS-, C-group) $\times 2$ (Word type: Numbers, Substances) $\times 2$ (Trial block: first half, second half) ANOVA with repeated measures. The Word type $[F(1,87)=36.86, P<0.001]$ and Trial block $[F(1,87)=21.76, P<0,001]$ main effects proved to be significant, showing a generally slower response to number words and a general acceleration of response as the experiment progressed. The Group main effect was not significant $[F(1,87)=2.90, n$.s.] indicating that the groups did not differ in mean response times. The crucial Group $x$ Word type interaction was significant $[F(1,87)=4.07, P=0.047]$. Neither the remaining two-way interactions, nor the three-way interaction of Group $x$ Word type $\times$ Trial block, were significant. Hence there was no evidence that the selective interference in the TS- 
group decreased as the experiment progressed. The reminder halfway through had worked well.

TS-group

C-group

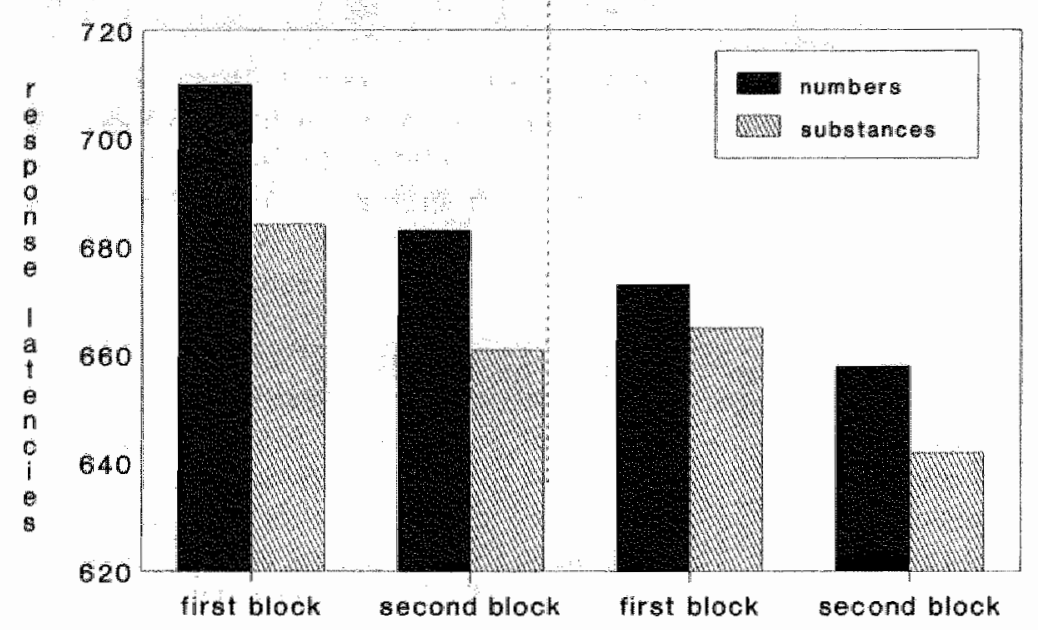

Figure 5.5.1: Mean response latencies for number and substance words per block

Planned comparisons of simple main effects (one-tailed tested) revealed that the graups did not differ significantly in response times for substance words $[t(87)=1.27)$, mean $_{\mathrm{Ts}}=672$ (s. $\left.d=69.1\right)$ msec, mean $_{\mathrm{C}}=654$ (s.d. $\left.=69.4\right)$ msec. As predicted, the TS-group was significantly slower than the C-group on number words $[t(87)=2.06, P=0.021]$, mean $_{\mathrm{Ts}}=696(\mathrm{~s} . \mathrm{d}=64.5)$ msec, mean $_{\mathrm{C}}=666(\mathrm{~s} . \mathrm{d} .=75.7)$ msec. Thus, the Group $x$ Word type interaction was merely due to a deterioration in the TS-group in colour naming number words, the stimuli to be suppressed.

\section{Discussion}

An experiment was conducted to decide whether cognitive avoidance is causal to attentional bias. Half the subjects were given thought suppression instructions, half were not.

The manipulation check showed that subjects in the TS-group indeed tried hard to suppress thoughts about numbers and to distract themselves from this subject. Despite their efforts, they were more distracted by the number words in the test and spent more time thinking of numbers than did the subjects in the C-group. Thought suppression therefore had a paradoxical effect, a nice replication of earlier findings (Lavy \& van den Hout, 1990 ). 
We used thought suppression as an experimental model for cognitive avoidance: It may be noted that the expression "cognitive avoidance" is a popular collective term used for various covert processes, such as repression, distraction, dissociation. rationalization and emotional numbness (Davis \& Schwartz, 1987; Foa \& Kozak. 1986; Foa, Steketee, \& Rothbaum, 1989b; Watts, 1990). But basically, cognitive avoidance may be conceptualized as either automatic $\mathrm{cq}$. unconscious or strategic cq. deliberate avoidance behaviour. The clinical relevance of unconscious cognitive avoidance has been emphasized since early Freudian formulations, but however interesting unconscious cognitive avoidance may be, it is difficult to investigate experimentally. On the other hand there are several findings which suggest that strategic cognitive avoidance is highly characteristic of anxiety disorders, especially of post-traumatic stress disorder and obsessive compulsive disorder. For post-traumatic stress disorder the association with strategic cognitive avoidance is obvious, even trivial, since one of the DSM-IIL-R diagnostical criteria of PTSD is "efforts to avoid thoughts or feelings associated with the trauma" (APA, 1987). But for other anxiety disorders too, there are subjective reports which suggest that anxious subjects deliberately attempt to avoid fear-arousing thoughts. For instance, Rachman and De Silva (1978) made up an inventory of normal and clinical obsessions; clinically obsessed subjects reported strongly resisting their obsessions, mostly by trying to distract themselves from the obsessive thought. Likewise, Craske, Street, Jayaraman and Barlow (1991) mentioned that subjects with panic attacks attempt to avoid thinking about bodily sensations that they feared. Even simple phobics appear to be reluctant to think about the phobic subject (Watts et al., 1986b). Clinically anxious subjects; then, report using avoidance strategies that closely resemble the thought suppression strategies of our experimental subjects. Thought suppression seems to be a relevant model for at least strategic cognitive avoidance responses shown by clinically anxious subjects.

The hypothesis that a strong motivation for cognitive avoidance causes an attentional bias seems to be confirmed by the Stroop response latencies; subjects in the TS-group showed a selective delay in colour naming number words. But before accepting this as evidence for a causal relationship between thought suppression and attentional bias, several critical points have to be dealt with.

Firstly, Stroop response latencies were in general larger for number words than for substance words. This finding is difficult to interpret. All subjects were students, and the general delay for number words might be the result of a greater familiarity with these words than with the substance words. But there is evidence that familiarity has no effect on response times (Mogg \& Marden, 1990), as can also be concluded from several therapy effects studies concerning attentional bias IFoa \& McNally, 1986; Lavy et al., 1993d; Watts et al., 1986a). Another explanation might be that the general delay with regard to number words results from priming effects, since prior to the Stroop task the cue "numbers" was explicitly mentioned four times to subjects in both groups. However, recent findings by Wegner and Erber (1992) provide evidence against the supposed priming effects. They offered normal subjects a very strong prime by explicitly instructing them to think about a certain topic while carrying out a modified Stroop task. These primed subjects showed an attentional bias neither towards that specific topic, nor towards words related to that topic. Thus neither familiarity nor pre-experimental priming seem to be convincing explanations for the general delay in colour naming number words. It remains unclear therefore why such a general effect was found. Whatever its origin, the 
general slowing down with regard to number words does not alter the conclusion that the TS-group displayed the predicted selective interference.

Secondly, the finding that the subjects in the TS-group showed a selective delay in colour naming suppression-related words may be open to alternative interpretations. Naturally, one might suggest that the attentional bias found may be due to the more demanding instructions the TS-group received. Subjects in the TS-group had to carry out two concurrent tasks, to suppress all thoughts about numbers and to colour name as quickly as possible, while subjects in the C-group only had to colour name. It may be argued that the TS group's deterioration in colour naming could be the result of the need to divide attentional resources between two tasks. Or, alternatively, subjects in the TS-group may have been brooding over their failures in suppressing all number thoughts while performing the colour naming task. These alternative interpretations of the data do not, however, seem to be valid, since the TS group did not show a general delay as can be concluded from the absence of a significant Group effect. The TS-group's deterioration in colour naming was specific for number words, and it is difficult to explain why subjects in the TS-group are particularly affected by number cues, without assuming that they are involuntarily distracted by the meaning of these stimuli.

An alternative interpretation of the selective delay in colour naming in the TSgroup is that this delay may not result from thought suppression directly, but from indirect processes initiated by the thought suppression instruction. More specificalIy, it is possible that thought suppression may result in an increase in suppressionrelated thoughts, and that subsequently the colour naming latencies of suppression. related words are affected. In other words, there is some question as to whether we found a direct effect of thought suppression, or allternatively, whether our findings simply reflect the fact that people selectively pay attention to cues that are relevant to their current interests and concerns. The results of several experiments make it rather doubtful as to whether people selectively pay attention to whatever subject is on their mind. Mogg and Marden (1989) showed that students who were active rowing club members for at least a year did not show an attentional bias towards words related to their favourite sport. And the above-mentioned study by Wegner and Erber (1992) provides even stronger evidence against the hypothesis that people selectively pay attention to stimuli associated to the topic that is on their mind. Our finding that the subjects in the TS-group showed an attentional bias for number words can therefore not simply be attributed to an increase in number* related thoughts.

To summarise, the attentional bias effects found in the TS-group do not seem to be the result of more demanding instructions and it is very unlikely that they merely reflect what people have on their mind. The thought suppression instruction thus appears to have specific effects on the allocation of attentional resources. Our findings go beyond the results of the Bentall and Thompson (1990) and Dawkins and Furnham (1989) studies. In these studies it was suggested that subjects who found the emotional words threatening made use of repression as a defence mechanism and showed an attentional bias. Both papers suggested that anxiety is the cause of both repression and attentional bias for emotional stimuli. The results of the present experiment however indicate that a motivation for cognitive avoidance by itself can produce an attentional bias, regardless of the emotional valence of the stimuli.

While results of the present study suggest that a strong motivation for cognitive avoidance may be sufficient $t_{i}$ it is not likely that it is necessary for attentional bias 
to occur. Data from several studies suggest that selective attention can also occur in favour of positively valenced stimuli (Channon \& Hayward, 1990; Lavy \& van den Hout, 1993a; Mathews \& Klug, 1993). It has frequently been suggested that selective attention to threatening stimuli may facilitate early detection of potential threat, and may thereby facilitate avoidance or escape from the threatening situation le.g. Lavy, van den Hout, \& Arntz, 1993e; Litz \& Keane, 1989; Macleod, Mathews, \& Tata, 1986; Mathews, 1989; Mogg, Mathews, \& Weinman, 1987). Perhaps this hypothesis can be broadened, and it can be hypothesized that attentional bias may occur for any stimulus that is of immediate personal relevance and elicits an urge to act, regardless of whether this urge is related to (cognitive or motoricl approach or to (cognitive or motoric) avoidance. Referring to the present experiment it may then be speculated that a strong motivation to avoid cognitively, elicited by a thought suppression instruction, will lead to an attentional bias for stimuli to be avoided and that this attentional bias may be adaptive in that it enables a subject to come up with a quick and adequate (cognitive) avoidance response. Of course the present data are insufficient to prove a functional relationship between an urge to act and attentional bias. However, the results of other studies also suggest that there might be such relationship (Lavy et al., 1993a; Lavy et al., 1993e). There is thus some tentative support for the hypothesis that an attentional bias can be shown towards any stimulus that requires a quick response from the subject. Whereas it is too early to embrace this hypothesis as being valid, it may offer a fruitful field for future experimental investigation. 


\title{
5.6 Attentional bias and facilitated escape: A pictorial test ${ }^{7}$
}

\begin{abstract}
Results of varilous experimental studies prove that fearful subjects show an attentional bias to threatening information. It is hypothesized that such attentional bias promotes facilitation of escape from potential danger. In this study, a paradigm is described in which both selective allocation of attention and facilitated escape can be studied in one pictorial task. Selective allocation of attention is assessed by measuring performance deterioration due to distraction with a threatening distractor. Facillitation is measured by the improved reaction time performance to threatrelevant cues. An experiment was conducted with 46 spider phobics. The results confirmed that phobic subjects show facilitated responses to threat-congruent cues. The potency of the pictorial task and possible relationships between attentional bias and avoidance are discussed.
\end{abstract}

\section{Introduction}

There is strong evidence that anxiety patients show ideosyncratic attentional biases to stimuli which are associated with threat. Selective allocation of attentional resources has been demonstrated with various experimental paradigms, like the modified Stroop task (e.g. Mogg, Mathews, \& Weinman, 1989), the dichotic listening task (e.g. Mathews \& MacLeod, 1986) and the probe detection task (e.g. MacLeod, Mathews, \& Tata, 1986). In general, responses are facilitated when attention can be focused on threat-relevant information (e.g. probe detection task), while responses are deteriorated when the subject has to ignore threat-relevant information and has to allocate attention to concurrent non-threatening information (like in the modified Stroop task). What could be the function of threat-relevant information prevailing over other kinds of information? One of the speculations is that an attentional bias for threat-related cues may be adaptive, in that it optimally prepares subjects to avoid or escape the feared object. Thus, attentional bias may enhance the early identification of threat-related stimuli, and facilitate avoidance or escape from potential danger.

Although the avoidance-hypothesis is brought to the front regularly (Litz \& Keane, 1989; Macleod \& Mathews, 1991; MacLeod et al., 1986; Mathews, 1989; Mogg, Mathews, \& Weinman, 1987), it has never been tested. A causal relationship between attentional bias and avoidance is difficult to prove, since it is hard to see 
how attentional bias can be independently manipulated. But if retarded response to threat-incongruent material and facilitated response for threat-congruent material could be demonstrated in one experimental paradigm, it would be an argument in favour of the hypothesis. The well-known paradigms mentioned above do not allow for investigation into an association between attentional bias and avoidance, therefore a new paradigm had to be explored.

An experimental paradigm through which facilitated and deteriorated responses can be examined during the same test is described by Von Streblow, Hoffmann and Kasielke (1985). They presented a series of slides to phobic subjects, each constructed from three pictures placed in a diagonal row: one central picture and two flankers. Phobic subjects had to decide as quickly as possible whether the centrall picture displayed an element from a class of objects mentioned shortly before. Response times were measured. Compared to slides with only neutral pictures, subjects were significantly slower in classifying the central picture if it was flanked by threatrelevant pictures. This can be interpreted as a manifestation of attentional bias. When however the central picture was threat-relevant and the flankers neutral, performance was better compared to slides with only neutrall pictures.

However suggestive, the data are difficult to interpret. Von Strablow et al. (1985) for instance did not mention the diagnosis of their subjects, used black and white pictures, did not report how (and how long) the slides were presented, how the response times were recorded etc.

We adopted the Von Streblow et al. paradigm to examine whether attentional bias and escape are associated. The predicted retarded responses to slides with threatening flankers would reflect pure selective allocation of attention to threatrelevant information. For threatening cues in the centre of the slides, the same attentional bias will result in a facilitated response. This predicted facilitated response is considered as an escape reaction, because in our test the slide disappeared immediately after the subject responded.

In an earlier experiment with a modified Stroop task (Lavy, van den Hout, \& Arntz, 1993d) spider phobics showed an attentional bias for spider words, which was reduced after a one-session exposure therapy. In the present study we used a comparable sample and offered an exposure treatment that was comparable with the earlier treatment. We predicted that, before treatment, spider phobics would show an attentional bias and a facilitated avoidance reaction, which would both be reduced after treatment.

In order to investigate possible test-retest effects, the spider phobics were randomly assigned to two groups. One group was tested twice, before and after treatment, the other group was only tested after treatment.

\section{Method}

\section{Sibjects}

Subjects were 46 spider phobics (all female, mean age 28.1 years, range 17.45 years), participating in a spider phobia research project of Limburg University. All the subjects met the DSM-III-R criteria for simple phobia. Two measures of spider phobia were used: the Spider Phobia Questionnaire (SPQ: Klorman, Weerts, Hastings, Melamed, \& Lang, 1974) and a behavioural approach task (BAT: Arntz \& Lavy, 1993a). SPQ scores range from 0 to 31 , with 31 as maximal self-reported 
spider phobia. During the BAT subjects were seated behind a large table $10.4 \mathrm{~m} x$ $3.0 \mathrm{~m})$, with a live spider in a closed glass jar at the far end of the table. Using a guiding string subjects had to pull the spider as nearby as they would normally tolerate. The BAT was scored on a 13 point scale from 0 (distance still $3 \mathrm{~m}$ ) to 12 (spider on the subject's hand).

The subjects received free treatment in exchange for their participation in the research project. Twenty-five subjects were assigned to the pre-post group (group A), and 21 subjects to the post-only group (group B).

\section{The slides}

Slides were constructed from phobic and neutral pictures. Phobic pictures were two different photographs of spiders, neutral pictures were photographs of a sheep and a duck. The neutral pictures proved to be non-emotional in pilot studies. Care was taken that the colours and brightness of the several pictures more or less corresponded, in order to minimize facilitated recognition by slide differences. Each silde was constructed from three pictures; the angle between the central picture and flankers was (on a $3 \mathrm{~m}$ distance from the subject) $26.5^{\circ}$.

Four types for slides were made: 12 slides with three neutral pictures (NNN), 12 slides with a neutral picture in the centre and two phobic flankers (PNP), 12 slides with a phobic picture in the centre and two neutral flankers (NPN) and 12 slides with only phobic pictures (PPP). Slides of the last type were only added to counterbalance the frequency of presentation of the various pictures, and were not included in the analyses. Therefore, they will not be discussed further.

A total of 36 experimental slides was thus presented to the subjects: 12 slides of each type. Because two meutral and phobic pictures were used, two variants were made of each slide type: the duck and the sheep, combined with each other or with spider 1 or 2 . The two flanking pictures were always identical.

In order to limit recognition, all combinations of pictures were presented on four different background colours (red, blue, yellow and green).

\section{Apparatus}

Subjects were tested individually in a sound attenuated room. They were sitting at a table, in front of them lay a booklet and three adjacent buttons. On each page the booklet had a simple statement about the slide to be presented: "It is a spider". "It is a duck" or "lt is a sheep". The left button was marked with "YES", the right button with "NO". The middle button was to be kept pressed during slide presentation, in order to secure the starting position before pressing the YES or NO button.

A Compur shutter, with an accuracy of $4 \mathrm{msec}$, assured the exact time of slide onset. Shutter times were directed by an Olivetti M250 microcomputer. Slides were projected $(1 \times 1.6 \mathrm{~m})$ by a Kodak Carousel onto a screen, approximally $3 \mathrm{~m}$ in front of the subject. Slide sequence was determined randomly, but the series was identical for all subjects.

\section{The pictorial task}

Before slide presentation, the subject read the relevant statement in the booklet and kept the middle button pressed. The subject was instructed to concentrate on the central picture, to ignore the flanking ones, and to decide as quickly as possible whether the statement was correct or incorrect for the central picture. For each slide type, half of the statements was correct and half incorrect. 
As soon as the subject decided whether the statement was correct or not; she had to release the middle button and press the proper button: YES or NO. Two reaction times were measured: the time needed to release the middle button and the time needed to press the proper button. Errors were marked and excluded from anallysis.

In the instructions, a lot of emphasis was put on the fact that the slide would disappear immediately after the subject had reacted. Thus the subject was aware that she could escape exposure to threat-relevant pictures by reacting quickly.

As soon as the subject had pressed one of the two decision buttons, the slide disappeared. A 6 seconds intertrial interval allowed the subject to turn over the page and to read the next statement.

\section{Manipulation check}

After the pictorial test, subjects filled in two Visual Analogue Scales about the emotionality of each picture. The pictures were now presented as $8 \times 12 \mathrm{~cm}$ photographs. The first question (VAS1) was, how emotional the picture was $10=$ not emotional at all, $100=$ extremely emotional), the second question (VAS2) what the emotional valence of the picture was $(0=$ very negative, $100=$ very positive).

\section{Procedure}

Subjects filled in the SPO and participated in the BAT. After the assessment, the pictorial task was introduced to the subjects in group A. The subjects were presemted ten slides with different pictures (of babies and men) in order to get used to the procedure. After the experimental pictorial test, the manipulation check was completed. All subjects were then offered two exposure in vivo sessions, with one week between the first and second sesision (for more details about the exposure procedure see Arntz \& Lavy, 1993a). After the second exposure all subjects were presented with the same pictorial test, followed by the manipulation check. Finally, subjects performed a post-treatment SPQ and BAT test.

\section{Results}

\section{Hypotheses were:}

1. Before treatment the spider phobics show retarded responses on slides with phobia-relevant flankers as compared to slides with neutral flankers.

2. Before treatment the spider phobics show facilitated responses on slides with a phobia-relevant centre.

3. Both the retarded and the facilitated responses are reduced after treatment, and these reductions are not due to test-retest effects.

\section{BAT and SPQ measurements}

Two measurements were used to determine whether the exposure treatment had been effective: the SPQ and the BAT. The BAT scores $[t(44)=18.76, P<0.001]$ and the SPQ scores $[\mathrm{t}(44)=13.16, P<0.001]$ improved both strongly. Neither before nor after treatment were there any differences between the $A$ and $B$ group $(P>0.50$ in all cases), thus the randomization procedure had succeeded. 
Manipulation check

Both the ratings concerning spiders and neutral pictures were combined; the mean VAS scores of the subjects are shown in table 5.6.1.

\begin{tabular}{lllll}
\hline & Spiders & & Sheeps \& & Ducks \\
\cline { 2 - 5 } & Emotionallity & Valence & Emotionality & Valence \\
\hline pre teat & $70.1(29.8)$ & $16.6(14.8)$ & $24.2(25.5)$ & $62.5(15.0)$ \\
post test & $479(33.0)$ & $39.4(20.9)$ & $23.0(21.5)$ & $64.0(14.9)$ \\
\hline
\end{tabular}

Table 5.6.1: Means and standard deviations of ratings on the Emotionality and Valence VAS scales

Before treatment, the spider pictures given a much higher emotional rating (VAS1) than the neutral pictures $[t(24)=-6.06, P<0.001]$. The spiders were rated negatively (VAS2), while the neutral pictures were considered as slightly positive $[\mathrm{t}(24)=10.57, P<0.001]$.

After treatment, the spider pictures were still given a higher emotional rating on the VAS1 [t $(45)=-5.57, P<0.001] 1$ and were rated more negatively on the VAS2 $[t(45)=6.28, P<0.001]$ than the neutral pictures. However, while the ratings of the neutral pictures did not change significantly, the spider pictures were given a lower emotional rating $[\mathrm{t}(24)=4.66, P<0.001]$ and were rated less negatively $[\mathrm{t}(24)=$ 5.01, $P<0.001]$ after treatment than before treatment.

The VAS scores indicate that the composition of the slides was adequate for the purpose of the study.

\section{The response times}

Two measurements were available: the response time needed to release the middle button (RELEASE) and the response time needed to press one of the decision buttons (PRESS). Analyses were carried out with both measurements; as the two measurements tend to produce identical patterns, the results of the PRESS data will be presented. Whenever the RELEASE data deviate from the PRESS data, the results of the RELEASE data will be mentioned too.

\begin{tabular}{llll}
\hline & NPN (s.d.) & NNN (s.d.) & PNP (s.d.) \\
\hline butore exposure lgroup A) & $918(306)$ & $983(310)$ & $989(326)$ \\
allter axposure (group A) & $818(244)$ & $829(245)$ & $852(252)$ \\
after exposure (group B) & $854(186)$ & $908(252)$ & $946(242)$ \\
\hline
\end{tabular}

Tab/e 5.6.2: PRESS response times - means (msec) and standard deviations

\section{Before treatment}

Because two specific effects were expected, data were tested with separated Analyses of Variance (ANOVAs), instead of combining the data in a repeated measures design. The pre-treatment data could only be submitted to a within subject design. Mean response times are shown in table 5.6.2. 
Compared with the reaction times on the NNN-slides, spider phobics were not slower in responding to the PNP-slides $[F(1,24)=0.14, \mathrm{n} . \mathrm{s}$.$] , and therefore no atten-$ tional bias in favour of the phobic flankers was found. But, as expected, they were quicker in responding to the NPN-slides $[F(1,24)=8.20, P=0.011$. Thus, as is shown in figure 5.6.1, although no distraction effect was found (hatched bars), the phobics showed a facilitated response when the spider was presented in the centre of the slide (black bars).

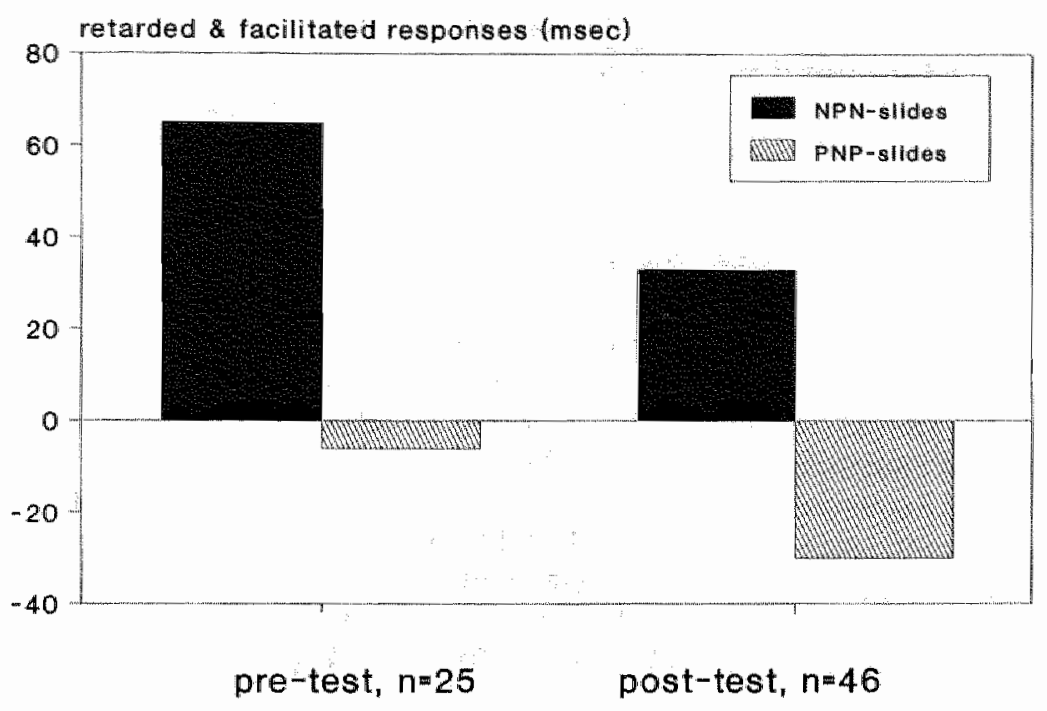

Figure 5.6.1: Retarded (below zero) and facilitated (above zerol responses

\section{After treatment}

In table 5.6.2 the mean response times after treatment are presented. ANOVAs were carried out to investigate differences within and between subjects. Compared with the reaction times on the NNN-slides subjects were now slawer on PNP-slides $[F(1,44)=4.01, P=0.05]$, and there was still a trend to be quicker on NPN-slides $[F(1,44)=3.59, P=0.07]$ (see also figure 5.6.1). When the RELEASE-data were analysed there was no facilitated response left after treatment $[F(1,44)=1.17$, n.s.l.

In order to investigate whether the pictorial test is susceptible to test-retest effects, 3 (Slide type: NNN, PNP, NPN) by 2 (Group: pre-post, post-only) ANOVAs were carried out. The two groups differed neither in retarded $[F(1,44)=0.28, \mathrm{n} . \mathrm{s}$. nor in facilitated responses $[F(1,44)=1.46, n . s$.$] , and although the post-only group$ tended to be somewhat slower in general, the difference was not significant $[F(1,44)=1.04, P=0.31]$. 


\section{Changes due to exposure treatment}

After treatment there were no differences between group $A$ and $B$; thus there is no evidence that the pictorial test is susceptible to test-retest effects. Alterations in the mean response times from the first to the second test can therefore be attributed to the exposure treatment. In accordance with the hypothesis, the facilitated response was significantly reduced $[t(24)=2.19, P=0,04]$. The response to the PNP-slides, which was expected to be retarded before treatment, did not change significantly $[\mathrm{t}(24)=-0.69, P=0.50]$ as a result of the exposure therapy.

To summarize the data: in agreement with the hypothesis the facilitated response shown before treatment was reduced (PRESS) or even eliminated (RELEASE) as a result of the exposure in vivo. Contrary to the hypothesis the retarded response which was not shown before treatment tended to occur after treatment, although the deterioration was not significant. There were no group differences, thus there is no evidence that the pictorial test is susceptible to test-retest effects.

\section{Discussion}

Aim of the present study was to investigate both retarded and facilitated responses, in order to test whether attentionall bias and escape are associated and may be causally related. The hypotheses were partly confirmed. Spider phobics showed a facilitated response when they had to respond to spiders and this facilitated response decreased as result of exposure treatment. From other experiments (e.g. Lavy et al., 1993d) it is known that spider phobics, performing a Stroop task, show retarded response latencies when presented with spider stimuli. In the present study no retarded responses for slides with spider fllankers were found in untreated phobics. Curiously, it was the treated sample that showed a significant degree of distraction by threatening flankers.

Why didn't untreated subjects show retarded responses when spiders were used as flanking pictures? One possibility is, that they only show retarded responses when they are primed for the threatening material. This post-hoc explanation could be tested, since the statement "It is a spider" preceded half of the slides with spider flankers. Analyses revealed no effect of priming. Besides this negative finding, priming effects would also leave unexplained the question why the untreated subjects reacted according to expectations when the spider was in the centre of the slide, the spot they were instructed to concentrate upon. The most likely reasion for the lack of distraction to the flanking pictures is, that subjects may have been able to ignore the flanking pictures because the angle between central and flanking pictures was relatively large ${ }^{B}$. Untreated phobics are very anxious and highly motivated to escape from spider cues, as can be gathered from the facilitated escape data. The wide angle set-up may have inspired untreated subjects to concentrate on the central picture and to deliberately ignore any (possibly aversive) flankers. The mild

Note that in other paradigms threat-relevant and task-relevant material coincide (Stroop tests) or are divided with only a small angle between them (probe detec. tion tasks; e.g. Macleod et al., 1986). In Stroop tests it is well known that inhibition decreases rapidly with increasing spatial segregation (see Glaser \& Glaser, $1989)$. 
anxiety remaining after treatment may be too weak to promote delberate ignoring of flanking pictures, but strong enough to promote selective allocation of attention to these flankers. In our previous study (Lavy et al, 1993d), the attentional bias effect was not completely eliminated after treatment either. The explanation, that in the present experiment an interaction effect exists between anxiety and the angle between the central and flanking pictures, is of course a post-hoc one, but it is amenable to experimental testing.

Though no attention distraction effects in untreated subjects are found in this study, there is ample evidence for such an effect in spider phobics (Watts, Mckenna, Sharrock, \& Trezise, 1986a; Lavy et al., 1993d), suggesting that our negative results in untreated subjects are indeed a result of task peculiarities. Taking the existence of an attentional bias in spider phobics for granted, what could be the relationship between attentional bias and escape? We hypothesized that attentional bias promotes facilitation of escape and we found some data to support this view. Meanwhile, the possibility remains that a third and independent factor causes both selective attention and a facilitated response. In fact, such an idea was put forward by Gray (1982), years before attentional bias became a popular subject in the literature. Conceptualizing a "Behavioural Inhibition System" (BIS) he claimed that both increased attention and arousal mediated avoidance are independent functions of the BIS. (Gray, 1982, p.12-13). While activation of the BIS is supposed to be restricted to "conditioned stimuli for punishment and non-reward", attentional bias can also occur for positive stimuli. Since hungry subjects show an attentional bias for food items which are positively evaluated (Channon \& Hayward, 1990; Lavy \& van den Hout, 1993al, activation of the BIS can be considered as a sufficient but not a necessary reason for the occurrence of attentional bias. Attentional bias is not limited to anxiety states, but can also occur in other emotional states. In this broader view, attentional bias may occur whenever immediate action is needed, irrespective of whether the action is approach-or avoidance directed (Lavy \& van den Hout, 1993a). The concept that the wish to act pertains to emotional states, is not a new one. According to Frijda (1986, 1989), emotions are characterized by action readiness and appraisal. For each emotion Frijda distinguishes a different mode of action readiness (action tendency), e.g. approach, avoidance and inhibition (Frijda, 19861. Although these tendencies are emotion-specific, the behaviour patterns needed to execute these tendencies can show some overlap; e.g. both the tendency to approach and to avoid stimulate motoric activation. Hyperattention to relevant stimuli could optimize quick execution of both activation tendencies.

The possibility that an independent factor (anxiety, BIS or the like) caused both retarded and facilitated responses, can not be ruled out with the pictorial paradigm as used in the present experiment. But, given that such a third variable is not a necessary condition for the occurrence of attentional bias, it may for reasons of parsimony be preferable to assume that selective allocation of attention promotes facilitated responding.

For research purposes phobic cues can be presented either in vivo, or in words (cf. Stroop, dichotic listening tasks, probe detection tasks etc.). Another approach, taken in the present experiment, is to offer cues in a pictorial way. One might think that a pictorial representation is more similar to reality (in casu a living spider) than a spider-related word. From that argument it would follow that using a pictorial paradigm the retardation and facilitation effects will be stronger than using a linguistic approach. Note however that results obtained with the present pictorial stimuli 
were less pronounced than with a Stroop task (Lavy et al., 1993d). The wide-spread use of Stroop tasks and related paradigms in anxiety research warrant study of the power and validity of linguistic, pictorial and in vivo stimuli. 


\title{
5.7 Selective attention evidenced by pictorial and linguistic Stroop tasks $^{9}$
}

\begin{abstract}
Attentional biases to threatening stimuli are frequently demonstrated in anxious subjects by means of various linguistic experimental tasks. We argue that, for reasons of both ecological validity and accessibility of affective information, a pictorial task would be a more appropriate test of attentional bias in spider phobics. Before and after a two-session exposure treatment, 27 spider phobics were presented with a combined pictorial/linguistic Stroop task. As hypothesized, spider phobics showed an attentional bias for pictorial and linguistic spider-related stimuli and both attentional biases were strongly reduced after treatment. In contrast to our expectations, pictorial stimuli generally resulted in smaller attentional biases than linguistic stimuli. Two post-hoc explanations are discussed.
\end{abstract}

\section{Introduction}

Since Bower (1981) wrote his influential paper about an associative network theory of emotion, a lot of experiments have been carried out to investigate the predictions derived from this model. According to Bower's theory, emotions are represented in mental networks of associations between memories, ideas and concepts. Activation of one node in the associative network results in a spreading of activation through which the associated nodes are triggered. Bower's theory predicts mood-congruent biases in every stage of information-processing. Subjects with emotional disorders would show a mood-congruent perceptual, attentional, interpretational and retrieval bias.

The mood-congruent attentional bias has been investigated extensively in anxiety patients. According to the associative network theory, they should attend selectively to threatening stimuli. The most widely used paradigm for measuring the allocation of attention is the modified Stroop task. Subjects are presented with words, printed in different colours. They are instructed to name the colours as quickly as possible, ignoring the meaning of the words. Because attentional resources are limited, the colour naming performance is supposed to deteriorate if the meaning of the word demands attention. The more the subject slows down in colour naming, the more attention is said to be allocated automatically to the meaning of the words. Usually an interference score is calculated by subtracting the mean response

9 This section is based on: Lavy, E.H., \& Hout, M.A. van den (1993). Selective attention evidenced by pictorial and linguistic Stroop tasks. Behavior Therapy (in press). The discussion of the present section is theoretically more elaborated than the article. 
latency for neutral words from the mean response latency for threatening words. When anxious and normal subjects are compared, anxious subjects typically show larger interference scores than do normal subjects. Selective interference effects have been demonstrated in subjects with generalized anxiety disorder (Magg. Mathews, \& Weinman, 1989), panic disorder (Ehlers, Margraf, Davies, \& Roth, 1988; McNally, Riemann, \& Kim, 1990b), rape victims with post-traumatic stress disorder ICassidy, McNally, \& Zeitlin, 1992; Foa, Feske, Murdock, Kozak, \& McCarthy, 1991), Vietnam veterans with post-traumatic stress disorder (McNally, Kaspi, Riemann, \& Zeitlin, 1990a), patients with obsessive compulsive disorder (Foa, llai, McCarthy, Shover, \& Murdock, 1993) and phobias (Hope, Rapee, Heim berg, \& Dombeck, 1990; Lavy, van den Hout, \& Arntz, 1993d; Watts, Mckenna, Sharrock, \& Trezise, 1986a). There is some evidence that the selective interference effects in anxious subjects can be significantly reduced as a result of behaviour therapy (Lavy et al, 1993d; Watts et al, 1986a). Although the selective interference effects are usually interpreted as selective allocation of attentional resources, alternative interpretations are brought to the front regularly. In particular the claim of automaticity is frequently refuted (for an extensive review about the interference effect, see Mactieod, 19911.

Several researchers reasoned that, if selective interference can be considered as an attentional bias towards threatening information, this would be consistent with clinical features of anxiety (MacLeod, Mathews, \& Tata, 1986; Mathews \& Eysenck, 1987; Mogg. Mathews, \& Weinman, 1987). Anxiety is characterized by a vigilant monitoring of the environment for future danger. An attentional bias for threatening information may enhance an early detection of potential danger "by which avoidance or escape reactions are facilitated (Lavy, van den Hout, \& Arntz, 1993e; Litz \& Keane, 1989; Mathews, 1989; Mogg et al., 1987).

Whereas various laboratory tests are carried out to model clinical phenomena. the difference between the experimental procedures and the modelled phenomena are impressive. For one thing, modified Stroop tests, dichotic listening tests and probe detection tests are all linguistic tasks; the stimuli presented in such tasks are merely words, referring to the feared objects or situations. It can be questioned whether this linguistic approach is always preferable. Of course, for example the written word spider would still produce some attentional bias in spider phobics. Not because the spider phobic would profit from an early detection of just a word, but because the word represents the feared object. A picture of a spider, however, does not only represent the phobic cue, but also shares perceptual characteristics of the feared stimuli. One might therefore argue that in case of spider phobia pictures will offer a better representation of real life stimuli, i.e. will have greater ecological validity, than words.

Recent studies of the Stroop effect provide another argument for non-linguistic stimulus presentation. Glaser and Glaser (1989) made a distinction between the semantic system and the lexicon; two functionally separate, but connected, systems. They stated that the semantic system controls the perception of objects/pictures and the execution of physical actions (like pressing keys), whereas the lexical system provides perception of language and controls written and spoken output. Response times are mainly determined by the length of the pathway between nature of input and required output. Subjects will, for example, be quicker to name rather than to categorize a word, since for word naming only the word nodes in the lexicon are activated after which responding is possible. But the pathway for categoriz- 
ing a word will start at the lexicon, after which a corresponding concept node in the semantic system can be activated, then the corresponding word node of the cat egory can be activated in the lexicon and finally the subject is able to respond (Glaser \& Glaser, 1989). Following the Glaser and Glaser (1989) model, and the De Houwer and d'Ydewalle (1992) modifications, De Houwer and Hermans (1992) argued that interference in response times will be found in a Stroop test whenever the target's pathway is of equal length or longer than the distractor's. In two inventive experiments, they found evidence for Bower's assumption that affective information is stored in the semantic system. One of their conclusions was that pictures can access affective information more directly than words can (De Houwer \& Hermans, 1992).

From both the argument of ecological validity and the argument of accessibility of affective information; the assumption can be derived that pictures of spiders elicit anxiety responses in spider phobic subjects. Results from various psychophysiological experiments indeed provide evidence that pictures of spiders can elicit an emotional response directly in spider phobics (e.g. Hare, 1973), even if conscious identification of these pictures is prevented (Öhman, 1993).

For reasons of both ecological validity and accessibility of affective information, a pictorial paradigm would offer a more appropriate test of attentional bias than linguistic paradigms. It is frequently suggested 1e.g. Litz \& Keane, 1989; Mathews, 1989; Mogg et a/., 1987) that attentional bias may be adaptive, in that it may enhance early identification of threatening stimuli, and may facilitate avoidance or escape from possible danger.

Lavy et al. (1993e) used a pictorial test to investigate both attentional bias and facilitated escape in spider phobics. Spider phobics were presented slides consisting of a central picture flanked by two distracting pictures. Subjects had to respond as quickly as possible to the central picture, ignoring the flanking ones. As soon as they reacted, the slide disappeared. Lavy et al. (1993e) predicted that responding would be facilitated when the central picture was a spider (facillitated escape), whereas retardation would be found when the spiders were the distracting stimulii (attentional bias). Although the predicted facilitated escape was found, they failed to demonstrate an attentional bias effect. Since the angle between the central picture and the flankers was about $26.5^{\circ}$, it was plausible that the spatial separation of central picture and flankers was too large to distract the subject from the centre [see Glaser and Glaser, 1989, for a summary of results that show how distraction decreases with increasing spatial segregation]. Merckelbach, Kenemans, Dijkstra and Schouten (1993), using a different pictorial attention task, also failed to find evidence for attentional bias in spider anxious subjects. In the latter study, subjects were not selected for spider phobia, but for a score above average on a 31 -item Spider Phobia Questionnaire (Klorman, Weerts, Hastings, Melamed, \& Lang, 1974). However, the mean SPQ score of their sample $(\mathrm{SPQ}=15.0$, s.d. $=2.81$ was much lower than the mean SPQ score of real spider phobics $(S P Q=23.4$, s.d. $=3.11$, as reported by Arntz and Lavy (1993a). It remains therefore unclear whether this failure to find an attentional bias is indeed due to spatial separation, as suggested by Merckelbach et al. (1993), or to an inadequate selection of subjects.

In view of the potential theoretical relevance of demonstrating an attentional bias for threatening pictorial stimuli, we decided to conduct another experiment with a pictorial paradigm, in which target and distractor are integrated as much as possible. In order to link up with the best-known linguistic test, we constructed a pictor 
ial variant of the Stroop test. Instead of coloured words, pictures were presented on coloured circles and subjects had to name the colours of the circles as quickly as possible. Parallel with the linguistic Stroop tests, it was expected that the presentation of threat-relevant pictures would interfere more with the colour naming task than the presentation of neutral pictures. An attentional bias in favour of threatening information would therefore be reflected by a deterioration in colour naming the circles around threat-relevant pictures, as compared with colour naming the circles around neutral pictures. The addition of trials with coloured words allowed for a direct comparison between the pictorial and linguistic stimuli.

We presented such a combined pictorialilinguistic Stroop task to a group of female spider phobics, before and after a two-session exposure treatment. In an earlier study with a linguistic Stroop task (Lavy et al., 1993d), we found that the pre-treatment attentional bias was significantly reduced after a one-session exposure treatment. Aim of the present study was thus two-fold: (1) to replicate earlier findings that spider phobics show an attentional bias to spider-related stimuli and that a two-session exposure in vivo treatment can reduce this attentional bias, and (2) to investigate the effects of a pictorial Stroop trials, as compared with the commonly used linguistic Stroop trials. We predicted that, before treatment, spider phobics would show an attentional bias in favour of spider cues on both the pictorial and the linguistic trials. As a result of the exposure treatment, we expected the attentional biases to be reduced. Finally, since pictorial stimuli are more similar to real spiders than linguistic stimuli, we predicted that the magnitude of the interference would be greater for threatening pictures than for threatening words.

\section{Method}

\section{Subjects}

Subjects were 27 female spider fobics, mean age 22.44 years [range 19 to 49 yearsl, who participated in a spider phobia research project of Limburg University. All subjects met the DSM-III-R criteria for simple phobia, as was determined after a structured interview made by telephone. Prior to participation, subjects with additional Ax I and Ax II psychopathology were excluded.

\section{Spider phobia measurements}

Both subjective anxiety and behavioural approach, measured before and after treatment, were used as indicators for spider phobia. Self-reported anxiety was measured with the Spider Phobia Questionnaire (SPQ: Klorman et al, 1974), a 31 . item questionnaire (range 0.31, with 31 as maximal spider phobia). Internal consistency and test-retest correlations of the SPO are high, whereas little common variance is shared with scales measuring other specific fears (Fredrikson, 1983; Klorman et al., 1974). In our previous research SPQ proved to be highly correlated with a standardized Behavioural Approach Test (Pearson's $r>0.80$ ), and significantly correlated with subscales of the Spider Phobia Beliefs Questionnaire (Arntz, Lavy, van den Berg, \& van Rijsoort, 1993b).

Approach was measured with a standardized Behavioural Approach Test (BAT). During this test, subjects were seated at a large table $(0.4 \times 3.0 \mathrm{~m})$ with at the far end a live spider (Tegenaria Atrica), of about $3 \mathrm{~cm}$ long, in a closed container. Using a guiding string subjects had to pull the spider as nearby as they could tolerate. 
When they succeeded in pulling the spider at distance Om (BAT $=11$ ), they were subsequently asked to open the container $(B A T=2)$, to touch the spider with a pencil for 1 minute $(B A T=3)$, to touch the spider with a finger for 1 minute $(B A T=$ 4) and to put the spider on the hand (BAT =5). If they failed to take a particular step or wanted to stop, the last completed step was taken as BAT score. Our previ. ous research evidenced that the BAT is responsive to treatment effects (Arntz \& Lavy, 1993a) and that the BAT scores correlate with SPQ scores and avoidance of the most feared situation, as assessed by the main phobia subscale of the Fear Questionnaire (Marks \& Mathews, 1979 .

\section{The exposure in vivo treatment}

The subjects were presented with an exposure in vivo treatment along the lines of Öst (1989). Two exposure sessions were given, each session lasted about two hours. The second session was exactly one week after the first one. Before the first session the exposure rationale was given and the main dimensions of the subject's fear, e.g. colour, size or speediness of the spider, were determined. In the two exposure sessions the phobics were gradually exposed to live spiders. Exposure exercises ranged from looking at a spider in a closed glass jar to letting the spider walk on the hands. If necessary, the therapist modelled the exercise. Many spider phobics hold irrational beliefs, like "the spider walks so rapidly, I can not keep pace with it". If it seemed indicated, small behavioural experiments were carried out in order to examine the accuracy of these beliefs. In the example mentioned above, the spider phobic was asked to put the spider on the ground and to try to walk at the same speed as the spider did (which was very slow indeed!). For more detailed information about the exposure treatment, see Arntz and Lavy (1993a).

\section{Apparatus}

Slides were projected $(0.90 \times 1.40 \mathrm{~m})$ by a Kodak Carousel onto a white wall, approximately $4 \mathrm{~m}$ in front of the subject. A Compur shutter, directed by a Compaq Deskpro 386/25e personal computer, assuring the exact time of slide onset. A Sony Cardoid Dynamic microphone F560, which the subject held in her hands, was connected to a voice level detector that stopped the computer"s clock at the initiation of the subject's vocal response. The personal computer recorded these response latencies with an accuracy of 1 msec.

\section{The slides}

There were four types of slides: (1) pictures of spiders, (2) pictures of chairs, (3) spider-related words, (4) chair-related words. There were six different variants for each slide type. Each was presented with two different colours (red, blue, vellow or green), resulting in 48 different slides. Each colour was used 12 times, and colours were matched across the phobic and neutral conditions.

For the picture slides, photographs of spiders and chairs were cut out and placed on coloured circles. At presentation the circles had a $62 \mathrm{~cm}$ diameter, the minimal size to close around all picture variants. In order to prevent selective attention to a specific spatial location of the circle, care was taken that the spiders were placed on the circles in different positions, i.e. horizontally, vertically, and diagonally in two directions. The spiders and chairs were matched for colour (brown and black) and picture complexity. 
For the word slldes, words were presented in exactly the same colours as used for the circles of the picture slides. Words were presented in $19 \mathrm{~cm}$ high Helvetica letters. Splider-related words were: SPIDER, WEB, ITCHING, CREEP, COBWEB and HAIRY, and taken from a former experiment with spider phobics (Lavy et al,, 1993d). Chair-related words, matched with the spider words for length and number of syllables in the Dutch language, were: SIT, CHAIR, UPHOLSTERY, ELBOW REST, SEAT and STOOL.

\section{The Stroop test}

The 48 different slides were presented twice, resulting in a 96 trial Stroop test $(24$ slides per slide type). Slide sequence was determined randomly, with the restriction that slides from a similar slide type could not occur more than twice in succession. The series was identical for all subjects.

Subjects were tested individually. They were informed that a series of slides would be presented, on which words and pictures alternated at random. They were told that the pictures would be presented on coloured circles and the words in coloured letters. They were instructed to colour name as quickly as possible, ignoring the (distracting) meaning of the cue presented. Errors, which were infrequent, were marked and excluded from analyses.

\section{Procedure}

The spider phobics completed the SPQ upon arrival and were then instructed about the Stroop test (see above). They were offered 20 practice trials with duckand table-cues, followed by the experimental Stroop test. After the Stroop test, subjects participated in the BAT and were given the first exposure session. Subjects returned after a week for subsequently the second exposure session, practice and experimental Stroop test, BAT and SPQ.

\section{Results ${ }^{10}$}

\section{Spider phobia measurements}

Before treatment, subjects were highly anxious, as indicated by their SPQ scores $\left(m_{\mathrm{spo}}=22.48, \mathrm{s.d}=3.85\right)$, which were comparable with those of earlier studies (Arntz \& Lavy, 1993a). They were hardly or not able to pull the spider in the closed container in front of themselves $\left(m_{\mathrm{BAT}}=0.56, \mathrm{s.d} .=1.29\right)$.

As a result of the exposure treatment, the SPQ scores declined $\left(\mathrm{m}_{\mathrm{SPO}}=8.80\right.$, s.d. $=5.99)$ and the BAT scores elevated $\left(m_{B A T}=4.80, s . d .=0.50\right)$, demonstrating a reduced anxiety and strongly improved approach behaviour. In fact, most subjects were now able to let the spider walk on their hands. The improvement was significant for both the SPQ score $[\mathrm{t}(24)=10.61, P<0.001]$ and the BAT score $[\mathrm{t}(25)=$ $15.92, P<0.0011$.

10 For both prem and post-tireatment there were same missing data. Before treatment, one subject consequently failed to colour name correctly the slides with spider pictures. And another subject did not return after a week. Only the data of 25 subjects, of which there was a complete dataset, are analyzed. 


\begin{tabular}{lllll} 
& \multicolumn{2}{c}{ Pictorial } & Stimuli & \multicolumn{2}{c}{ Linguistic Stimuli } \\
\cline { 2 - 5 } & Spiders & Chairs & Spiders & Chairs \\
\hline Pre-treatment & $680(70)$ & $655(67)$ & $789(119)$ & 7331981 \\
Post-treatment & $644(66)$ & $640(67)$ & 718187 & $687(70)$ \\
\hline
\end{tabular}

Table 5.7. 1: Response times: means (in msec) and standard deviations (im parentheses)

\section{Stroop trials}

The mean response times were analyzed via a threeway ANOVA with repeated measures: 2 (Stroop task: pictorial, linguistic) $\times 2$ (Time: before, after treatment) $x$ 2 (Stimulus: spiders, chairs). The mean response times are presented in table 5.7.1, the main and interaction effects obtained by the $2 \times 2 \times 2$ ANOVA are summarized in table 5.7 .2 .

The main effect of Stimulus showed that the phobic subjects generally colour named the spider stimuli slower than they did the chair stimuli. The Time main effect was also significant and displayed that subjects were generally faster in completing the second test than the first test. The significant Stimulus $x$ Time interaction provided evidence that the acceleration shown in the second test was more prominent for spider stimuli than for chair stimuli. There was no significant threeway interaction, thus the two types of Stroop tasks showed a comparable course in time. In other words: for both the pictorial and the linguistic Stroop trials the deterjoration in colour naming the spider stimuli was reduced as a result of two sessions exposure in vivo.

\begin{tabular}{lcccc}
\hline & Mean square & DF & $F$ & $P$ \\
\hline Stroop task & 274675.89 & 1.24 & 88.00 & 0.000 \\
Time & 79535.94 & 1.24 & 12.20 & 0.002 \\
Stimulus & 42596.28 & 1.24 & 39.52 & 0.000 \\
Stroop task $\times$ Time & 10565.89 & 1.24 & 10.60 & 0.003 \\
Stroop task $\times$ Stimulus & 11488.21 & 1.24 & 11.16 & 0.003 \\
Time $\times$ Stimulus & 5994.17 & 1.24 & 9.24 & 0.006 \\
Stroop $\times$ Time $\times$ Stimulus & 18.47 & 1.24 & 0.04 & 0.836 \\
\hline
\end{tabular}

Table 5.7.2: ANOVA with repeated measures for the response times with three within-subjects factors (Stroop task, Time, Stimulus)

The data obtained with the pictorial and linguistic stimuli are presented in figure 5.7.1 and are also analyzed separately. Consistent with our hypothesis, before treatment subjects responded significantly slower to spider stimuli than to chair stimuli on both the pictorial $[t(24)=3.29, P=0.0031$ and the linguistic Stroop trials $\llbracket t(24)=4.88, P<0.001]$. After treatment, on the pictorial Stroop trials there was no difference in response times to spider and chair pictures $[4(24)=0.95, P=0.3521$, whereas on the linguistic Stroop trials subjects still responded slower to spider stiuli than to chair stimuli $[\mathrm{t}(24)=4.50, \rho<0.001]$. Compared with the pre-treatment 
measures, the selective interference was significantly reduced in both the pictorial trials $[t(24)=2.35, P=0.027]$ and the linguistic trials $[t(24)=2.38, P=0.026]$.

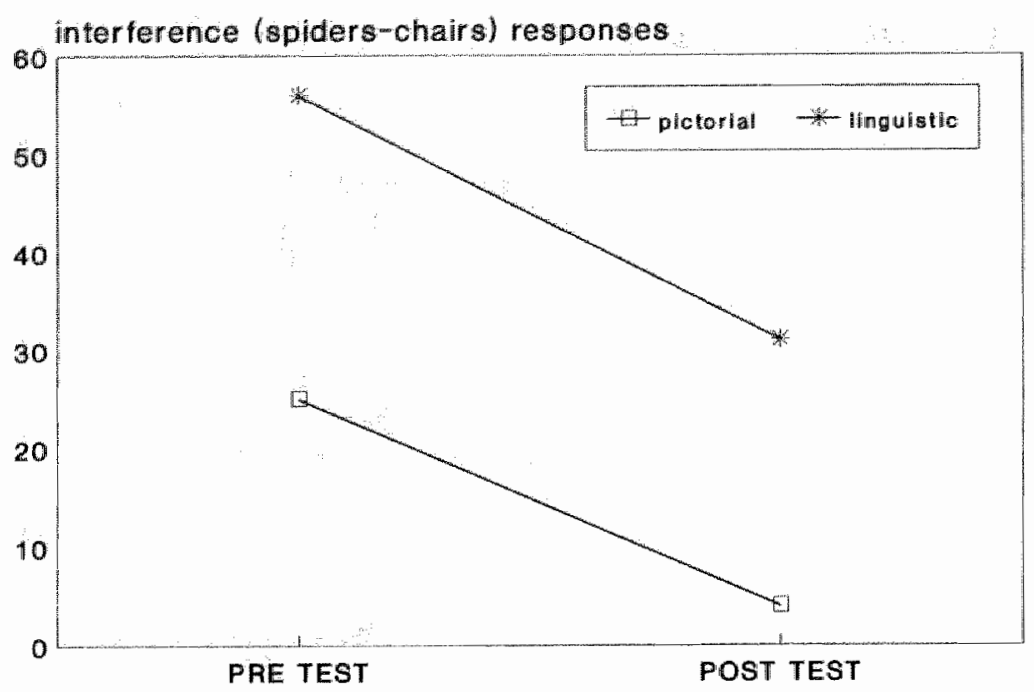

Figure 5.7.1: Interferences in linguistic and pictorial trials, before and after treatment

The linguistic and pictorial stimuli compared

As can be seen in table 5.7.1 and table 5.7.2, subjects responded generally more rapidly to picture slides than to word slides (main effect of Stroop task), showing that linguistic stimuli generally produce more interference in the colour naming task than pictorial stimuli. Besides, the significant Stroop task $x$ Stimulus interaction revealed that the selective interference in colour naming spider cues was more pronounced in the linguistic trials than in the pictorial trials. Since the subjects were generally more deteriorated in colour naming linguistic stimuli, these selective interferences are difficult to compare directly.

In order to relate the selective interferences to the mean response times for respectively pictorial and linguistic stimuli, relative interference scores were calculated. The selective interference for pictures was divided by the mean response time for pictures and the selective interference for words was divided by the mean

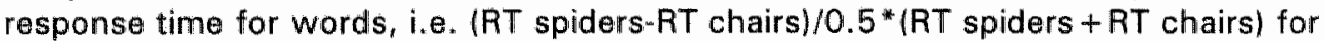
both pictorial and linguistic stimuli. These relative interferences differed significantly before $[\mathrm{t}(24)=2.12, P=0.045]$ and after therapy $\llbracket \mathrm{t}(24)=3.35, P=0.003]$, displaying a larger relative attentional bias in linguistic trials than in pictorial trials both before and after treatment. In other words, even when the longer mean response latencies to linguistic trials are taken into account, the selective interference of linguistic trials is significantly larger than the selective interference of pictorial trials. 
Curiously, Pearson"s correlations revealed that neither before $(r=0.11, n . s$.$) , nor$ after treatment $(r=0.07, n . s$.$) the attentional biases for spider words and pictures$ are correlated. Consequently, there seems to be no (linear) relationship between the linguistic and pictorial attentional bias. Closer inspection of the pre-treatment data revealed that the correlation was suppressed by three subjects, showing a strong linguistic and no pictorial attentional bias. When these three subjects were excluded from analyses, the pre-treatment correlation was $0.60(n=22, P<0.011$. Exclusion of these three subjects did not influence the results of the ANOVAs described above. The subgroup of three subjects was too small to allow for statistical testing. but on cursory inspection the SPQ and BAT scores of these subjects did not seem to be different from those of the other phobic subjects.

\section{Discussion}

From previous Stroop research it is well-known that spider phobics show an attentional bias for spider-related words. The present study was conducted in order to find out whether they also show an attentional bias for pictures of spiders and how the attentional biases for pictorial and linguistic information are compared.

Subjects were generally slower in colour naming linguistic trials than pictorial trials. This finding is consistent with earlier experiments fe.g. Klein, 1964; La Heilj, 1988) in which it was demonstrated that word distractors produce more interfer." ence than nonlexical distractors, and can be explained by the Glaser and Glaser (1989) and De Houwer and d'Ydewalle (1992) hypothesis. According to Glaser and Glaser (1989), colours are very simple pictorially represented objects. The pathway of colour naming will therefore start with the semantic system, then the corresponding word node in the lexicon will be activated and the colour can be named. Distracting pictures will follow a similar pathway, therewith causing little interference. But since distracting words have direct entrance to the lexicon, they have a very

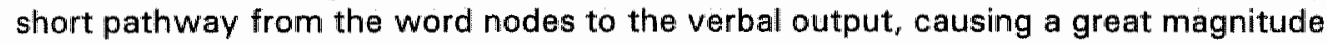
of interference with the colour naming task.

As hypothesized, before treatment spider phobics showed an attentional bias for pictorial and linguistic spider-related cues. As a result of two sessions of exposure treatment, both the linguistic and the pictorial attentional bias were significantly reduced. Therefore two out of three hypotheses are confirmed. But there are several reasons to reject the third hypothesis that is, that the pictorial attentional bias for spider cues would be greater than the linguistic one. In fact, the data of the present study show the opposite to be the case: selective interference, the measure of attentional bias, was greater for linguistic than for pictorial stimuli. This was observed both before and after treatment. Thus the spider phobics were relatively more distracted by spider-related words than by spider pictures. From the correlational data can be concluded that before treatment, for most phobics there is a fair correlation between the two attentional bias scores. A small but substantial group, however, showed only a (strong) linguistic attentional bias. Since the pictorial attentional bias is eliminated as a result of exposure therapy, the lack of significant correlations after treatment can be expected.

As expounded in the Introduction section, a pictorial task for attentional bias appears to have a greater ecological validity than a linguistic task. However, in contrast to the hypothesis, the attentional bias effect is not tempered but increased 
by the use of linguistic stimuli. What could be the reasons for this unexpected finding?

Firstly, it should be noted that the Glaser and Glaser (1989) theory is insufficient for understanding how linguistic stimuli can produce an attentional bias effect. If the linguistic information would interfere directly with the colour naming task, without activation of the semantic network, the conceptual meaning of the stimuli would not be activated and, consequently, selective interference for threatening stimuli would be impossible. Thus, for understanding the attentionall bias effect, it is necessary to presume that, as a linguistic Stroop trial is presented, there will not only be a direct lexical pathway, but also an indirect pathway via the semantic system. Such suggestion is not new, for instance Roelofs (1992) proposed a model of mental processing in which a written word was held to activate both its conceptual identification (cf. "semantic system') and its articulatory programme (cf. 'lexical systemi). Although there is some evidence that a word can be read aloud without activating the semantic system (e.g. Glaser \& Düngelhoff, 1984) there are also contradicting findings. De Houwer and Hermans (experiment 2, 1992) reported that distracting pictures interfered with word reading times; this would be impossible if the word was just lexically processed. Moreover, the results of severall studies suggest that the semantical appraisal is even activated before words are fully processed in the lexicon. Marcel (experiment 1, 1983) described a masking experiment, in which subjects were not able to guess correctly above chance whether the mask was preceded by a target or not. Gradually decreasing the target presentation times further, he found that subjects were longer able to guess correctly the meaning of the word than the graphic characteristics. Marcel (experiment 3, 1983) also found the original Stroop (incongruent colour) interference effect, even when the colour words were masked backwardly to prevent conscious awareness. In comparable masked modified Stroop experiments, it was shown that high trait anxious subjects still have an attentional bias for threatening words (MacLeod \& Rutherford, 1992) and that both trait and state anxiety are significantly correlated with selective interference from threatening distractor words (MacLeod \& Hagan, 1992). Thus, there is considerable evidence for automatic semantic processing of linguistic information.

Although a second pathway via the semantic system is a necessary condition for selective interference to occur, it is not sufficient to explain why, compared with the pictorial variant, the relative interference is increased in linguistic trials. The studies mentioned above suggest that a threat-relevant word can activate two different pathways, so that activation can be split in two directions as the lexical node is triggered. There will be one pathway directly to the lexical output, and another pathway to the corresponding semantical node and therewith to the affective information of the word presented. Assuming that each step in the pathway costs about an equal amount of time, it could be expected that the semantic connotation has just been triggered the moment the subject has to select the correct response and name the colour of the word (see figure 5.7.2). 


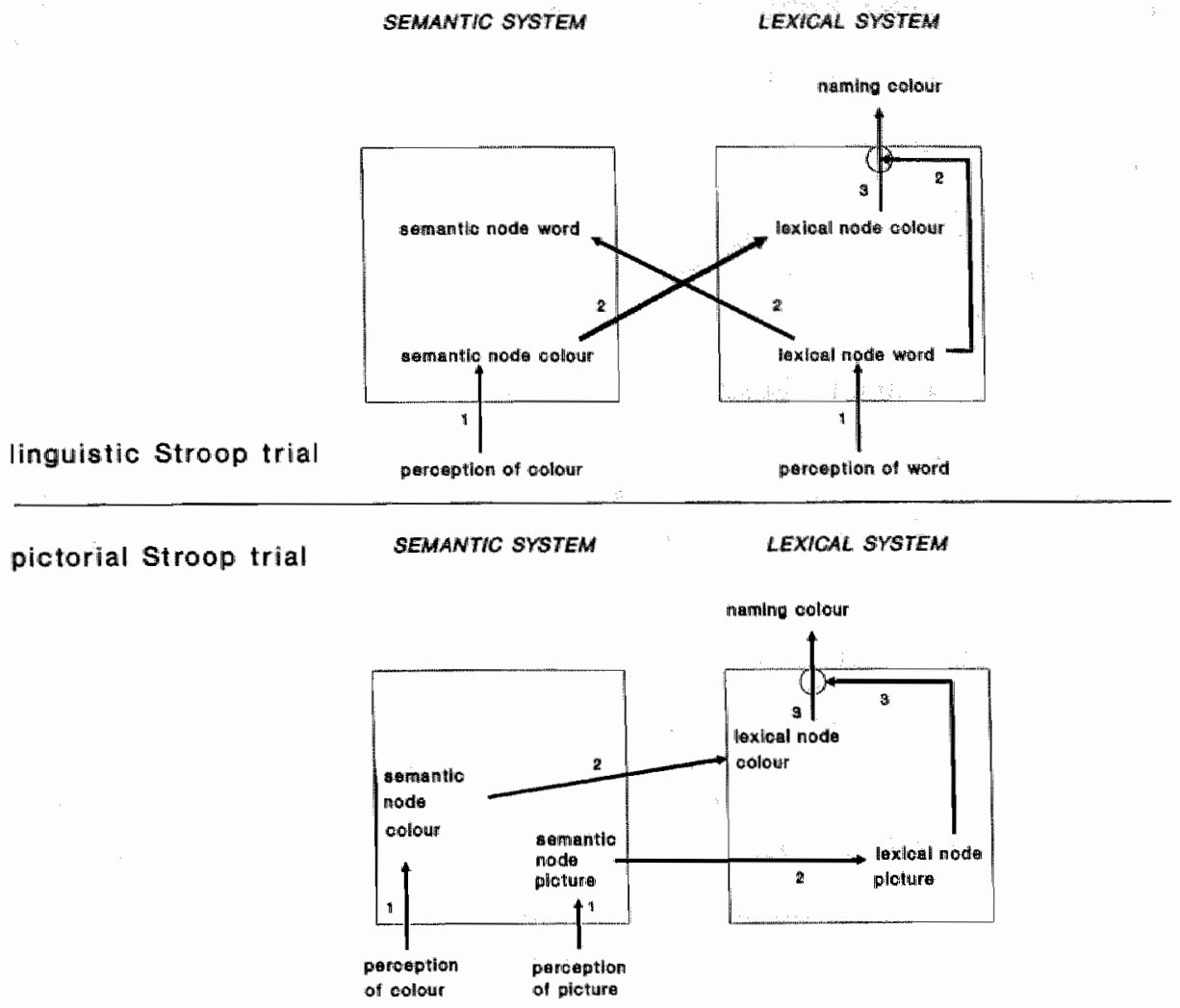

Figure 5.7.2: Model of processes involved in colour naming linguistic and pictorial tasks 
An anxious subject will attend selectively to the threatening meaning of the word, by which the colour naming performances will deteriorate. On the other hand, when the subject has to colour name a threatening picture, the semantic connotation of both the colour and the picture will be triggered at the same time (see also figure 5.7.21, after which both the target and the distractor will activate the lexical node. At the time the subject has to select the correct response and is able to colour name the picture, the semantic meaning of the picture is already waning in favour of the lexical activation. By that the colour naming interference may be smaller for pictorial stimuli than for linguistic stimuli. This post-hoc explanation can be tested with an experiment in which the stimulus onset asynchrony (SOA) is varied. For colour naming words, the maximall interference would be expected when the distracting word is presented just before or simultaneously with the colour to be named, whereas the maximal interference for colour naming pictures would be found when the picture is presented after colour onset.

A second post-hoc explanation is that linguistic stimuli can activate more semamtic nodes than pictorial stimuli, because words are less circumscript than pictures. While the perceptual characteristics of a spider picture limit the possible associations, therewith reducing the spreading of activation, the word "spider" can for instance refer to a (1) big/small (2) hairy/bald (3) dangerous/ innocuous (4) fast running/quietly sitting (5) black/light-coloured spider with (6) long/short legs (7) enormous/no jaws (8) in the subject's environment/far away and (9) in an attacking position/unaware of the subject. The absence of a concrete spider may also enhance polarization; Tesser (1976), experimenting with normal subjects, found that imaginary exposure to moderately (dis)liked objects results in more extreme associations than exposure to the real object. It is known that anxious subjects are, more than normal control subjects, likely to interpret linguistic ambiguous information as threatening (Butler \& Mathews, 1983; Mathews, Richards, \& Eysenck, $1989 \mathrm{~b})$. In this way anxious subjects, confronted with potential threatening stimuli, but lacking plain information that restrict mental processing, tend to polarize their associations even more than normals do. To stay with the earlier example, this means that, in absence of realistic information, a spider phobic may associate the word "spider" immediately with a big, hairy, black and long-legged monster, which is ready to jump on her the very moment. In sum, linguistic stimuli would trigger (1) a larger number and (2) more extreme, thus more threatening, semantic nodes, therewith producing more colour naming interference for phobia-related information than do pictorial stimuli.

It would be of interest to investigate this second option, since it would shed a different light on earlier attentional bias studies. As explicated in the Introduction section, a pictorial Stroop test is ecologically speaking a more valid test of the attentional processes which occur when a phobic subject is confronted with a threatening stimulus. Supposing the attentional biases, found in linguistic Stroop experiments, are indeed inflated by mental polarization effects, what is then the significance of these linguistic tasks? Although linguistic tests may be no pure tests of attentional bias effects, they may be clinically relevant.. From clinical practice we know that, in natural environments, phobic subjects frequently anticipate confrontation with the feared stimulus. A spider phobic may, in absence of a spider, for instance ruminate about the extremely threatening spiders that may appear when she is alone, or driving a car. From this point of view, the results obtained with 
linguistic tasks may not only reflect attentional processes, but also anticipatory overpredictions of threat.

In this study, we investigated a pictorial Stroop task and compared it to a linguistic task. Two post-hoc explanations are given for the unexpected finding that with the pictorial task, although more similar to the in wivo confrontation with a spider, smaller attentional biases are found than with the linguistic task. This matter can not be solved in the present study, but, in view of its theoretical consequences, replication and experimental dismantling the effect is warranted. 



\section{Chapter 6}

\section{Recapitulation and general discussion}

In chapter 3, several topics of interest and some gaps in our knowledge of the nature and function of attentional bias were reviewed. In chapter 4 , an overview was given of the main topics of our investigations, while the studies themselves were described in chapter 5. In the present chapter, the general discussion, the relevance of our experiments to broadening the extent of our knowledge will be considered. Therefore, issues raised in chapters 3 and 4 will be recapitulated upon and the contribution of our findings will be discussed. At the end of this chapter, a speculative integration of our findings is proposed and theoretical and clinical implications are discussed. 


\subsection{Replications of earlier findings}

As stated in chapter 4 , our first aim was to replicate the attentional bias effects in anxious subjects. Spider phobics (see section 5.1) were presented with a computerized Stroop task and were found to show a significant attentional bias for spider words. Using a card Stroop comparable results were obtained from obsessive compulsive subjects (see section 5.3): compared to normal controls the obsessive compulsive patients selectively attended to negative obsessive compulsive-related words. Exact replications of previous findings may not be earth-shattering, especialIy since attentional biases towards threat-related cues have been demonstrated frequently (see section 3.3), but these replicative findings formed the basis for the investígation of more refined and speculative hypotheses.

\subsection{The reactivity of attentional bias: Transient or enduring phenomena}

In section 3.4 it was argued that the role of trait anxiety in the occurrence of attentional bias is obscure, since there are both clinical and conceptual arguments that trait anxiety is interwoven with transient factors like state anxiety. In addition to these conceptual problems, several arguments against the notion that attentional bias can be explained by high trait anxiety were presented in section 3.4. The results of treatment studies form the basis of one of the arguments against the trait anxiety hypothesis. Foa and McNally (1986) and Watts et al. (1986a) showed that the attentional biases of obsessive compulsive patients and spider phobics were strongly reduced after behavioural treatment. If symptomatic treatment results in decreased selective attention effects, these effects have probably more to do with specific frightening concerns than with general trait anxiety. However, the Foa and McNally (1986) and Watts et al. (1986a) treatment programmes lasted several weeks and accounted for much more than a simple reduction in current anxiety (see also section 5.11).

In chapter 5, three experiments are therefore described in which spider phobics are tested before and after a one- (section 5.1) or two-session (section 5.6 and section 5.7) exposure in vivo treatment. The time-limited and circumscript qualities of the exposure in vivo treatment allowed for testing the pure effects of reduced current anxiety. In the section 5.1 and 5.7 experiments, it is demonstrated that spider phobics have an attentional bias towards spider-related cues before treatment, and that the attentional bias is reduced or even eliminated after one or two treatment sessions. It was mentioned in section 3.3 that attentional bias is a relative stable effect as long as the subject remains anxious. On the basis of the section 5.1 and 5.7 findings, it can be added that as soon as current anxiety is reduced, the attentional bias in favour of threatening information will also decrease.

Such strong reactivity of attentional bias effects is in conflict with the hypothesis that attentional bias reflects continuous personality traits. It is therefore highly unlikely that trait anxiety in itself plays an important role in the allocation of attentional resources. The question of transient or enduring factors will be discussed in more detail in section 6.8 and subsequent sections. 


\subsection{Stimulus familiarity}

Since it seemed to be clear from former research, reviewed in section 3.5 , that famillarity of the stimuli is not sufficient to explain the attentional bias effects, this issue was not investigated further in the chapter 5 experiments. However, it is notable that our data is consistent with previous findings. In two experiments, described in sections 5.1 and 5.7 , spider phobics showed an attentionall bias for spider stimuli before treatment, and this attentional bias was strongly reduced or even eliminated after treatment. Since the exposure in vivo treatment definitely familiarized subjects with spider cues, the reduction in attentional bias contradicts the familiarity hypothesis. Besides, in the two experiments in sections 5.3 and 5.5, normal subjects were randomly assigned to experimental and control groups. Group differences in attentional deployment could therefore not realistically be attributed to differences in stimulus familiarity. Thus, our data supports the conclusion in section 3.5 that familiarity cannot account for the attentional biases found in anxious subjects.

\subsection{Stimulus specificity}

Martin et a/. (1991) suggested that anxious subjects do not have an attentional bias towards threatening stimuli specifically, but towards all stimuli that are emotionally valenced (see section 3.6 for more details).

In the sections 5.1 and 5.3 experiments, attentional bias was determined by comparing the response latencies for relevant stimuli with the response latencies for both neutral control stimuli and emotional control stimuli. In section 5.3 , normal subjects were presented with food-, tool-, and holidlay-related stimuli. Half of these subjects had fasted for 24 hours preceding the Stroop task, and this manipulation resulted in a positive evaluation of food stimuli. Although rating scale data showed that fasting subjects evaluated food words as positively as holiday words, on the Stroop task they attended selectively to the food stimuli. Rejecting the emotionality hypothesis in normal subjects, however, does not imply that the Martin et al. (1991) hypothesis is not applicable to anxious subjects. In section 5.1, an experiment was described in which spider phobics and nonanxious controls were presented with spider words, mixed negative words and mixed neutral words. The results clearly demonstrated that spider phobics showed a selective attentional bias towards spider-related stimuli. However, as mentioned in chapter 4 " we did not check whether the enotional valences of the mixed negative words were as intense as those of the spider-related words, preventing definite conclusions being made about the selectivity of the attentional bias effect. Pre-experimental emotionality ratings indicated that the emotional control words were fairly negative (mean score $=2.2$ on a 10 -point scale, from $1=$ very negative to $10=$ very positivel. But theoretically the possibility remains that spider phobics would evaluate spider words even more negatively than the negative non-spider words.

Martin et al. (experiment 4, 1991) did check for the intensity of emotional valence, and found that anxious subjects allso showed an attentional bias towards positive stimuli which were as emotional as the threatening stimuli. However, as explained in section 5.2, Martin et al. (1991) used several antonyms for threatening 
words and, although not threatening, these words were certainly related with the concerns of anxious subjects. Three hypotheses about the selectivity of attentional bias effects were therefore postulated: the threatrelatedness hypothesis, the emotionality hypothesis and the concern-relatedness hypothesis. In the experiment described in section 5.2, obsessive compulsive patients and normal controls were presented with a Stroop test. In order to determine which of the hypotheses were supported by the findings, the Stroop test included five word categories: neutral words, negative obsessive compulsive words, positive obsessive compulsive words, general positive words and general negative words. Words in the four emotional categories were comparable in terms of intensity of emational valence. Obsessive compulsive patients only showed a clear attentional bias towards negative obsessive compulsive-related words, thus providing evidence for the threat-relatedness hypothesis. Since the obsessive compulsive patients did not selectively attend to positive obsessive compulsive-related words, no evidence was found for the hypothesis that anxious subjects show an attentional bias towards all stimuli associated with their fearful concerns. This finding was in contrast to the results of a comparable study by Mathews and Klug (1993), who found that a mixed group of anxious subjects showed an attentional bias towards positive anxiety-related words. Several potential reasons for this contradiction are given in section 5.2. Regarding Martin et al.'s (1991) emotionality hypothesis, we found in our study only a marginal effect of general emotional (positive and negative) words. From this and other emotionality studies le.g. Mathews \& Klug, 1993; McNally, Riemann, Louro, Lukach, \& Kim, 1992) it can at least be concluded that word sets with similar emotional intensities do not necessarily cause similar attentional biases in anxious subjects, and that emotionality, in itself, can thus not be a crucial factor in explaining attentional bias in anxious subjects.

\subsection{Memory bias}

In sections 2.6 and 3.7 it was reported that anxious subjects, who show an attentional bias towards threatening information, do not show an associated memory bias. It was suggested that this lack of evidence for an associated memory bias may be due to secondary avoidance responses, which prevent elaboration and storage of threatening information. In chapter 4 , it was argued that if this suggestion is correct, one might expect that an attentional bias towards positively valenced information will be associated with a memory bias towards that particular information.

This hypothesis was investigated in section 5.3. The food words were evaluated by subjects who had fasted, as being very positive cues. After finishing the Stroop task, however, they did not remember food words any better than did controls who had not fasted. Since evidence for an attentional bias in favour of food words was rather weak, these findings are insufficient to refute the suggestion that the lack of memory bias is due to avoidance responses. Firm conclusions about this hypothesis are thus still precluded. 


\subsection{Ecological validity of attentional bias tests}

In section 3.9 it was suggested that pictorial tests may offer more valid repre: sentations of the feared stimuli than linguistic tests. In two experiments spider phobics were presented with pictorial tests. In the section 5.6 experiment a paradigm was used that was derived from a study by Von Streblow et al. (1985). Subjects were presented with a series of slides, made up of three diagonally presented pictures. Before slide onset, they read a simple statement about the slicte to be presented. As soon as the slide appeared, the subjects had to decide as quicklly as possiblle whether the given statement was correct or incorrect for the central pic ture, and they had to press the appropriate (i.e. YES or NO) button. In order to promote quick response, the subjects were instructed to concentrate on the central picture and to ignore the flanking ones. Thus, making a decision about the central picture was the primary task and the flanking pictures served as distracting cues. It was hypothesized that spider phobics would be unable to ignore pictures of spiders and that they, consequently, would respond slower to nonthreatening central pictures which were flanked by pictures of spiders. The results yielded by this paradigm were confusing. Untreated spider phobics did not show retarded responses to slides with spider flankers, but the treated phobics were significantly more distracted by spider flankers than by neutrall flankers. The most plausible explanation for these findings is that the spatial segregation of the central and flanking pictures was large enough to allow for concentration on the central picture alone. In linguistic Stroop tasks it is difficult to ignore the phobic cues, because of the complete spatial integration of primary and distracting information, but in the flanking paradigm, primary and distracting cues are presented separately. Spatial segregation could account for the puzzling findings in the section 5.6 experiment.

In order to overcome the problem of spatial segregation, primary and distracting cues were integrated as much as possible in the 5.7 experiment. Spider phobics were presented with pictorial and linguistic Stroop trials. Pictorial Stroop trials consisted of pictures, presented on coloured circles. As in linguistic Stroop trials, the subjects had to name the colours as quickly as possible and to ignore the distracting stimuli (i.e. pictures). On both the linguistic and the pictorial Stroop trials, untreated spider phobics selectively attended to spider-related stimuli. After treatment, both the linguistic and the pictorial attentional biases were significantly reduced. Contrary to our expectations, pictorial stimuli generally resulted in smaller attentional biases than linguistic stimuli. These results should be considered with some care, since in the pictorial Stroop trials distracting stimuli and colours were not completely integrated (as they were in linguistic Stroop trials). However, in contrast to the potential alternatives, the present tests at least secured optimal, separate presentation of the linguistic and pictorial trials. And, as argued in section 5.7, several precautionary measures prevented subjects from concentrating on a specific, nondistracting loca. tion in the pictorial trials. If the smaller attentional bias obtained by the pictorial tests is regarded as a valid finding, several interesting theoretical options are left open. One possible explanation, given in section 5.7, is based on the Glaser and Glaser (1989) model of semantic and lexical systems. Following this schematic model, assumed pathways between input and required output will lead to distinct predictions about the interference caused by pictorial and linguistic stimuli. The reason why pictures are less distracting than words may be that the pathways followed after picture presentation result in less semantic and lexical activation at 
the moment of colour naming (see figure 5.7 .2 ). A second possibility, suggested in section 5.7 , is that linguistic stimuli can activate a larger number and more threatening set of cues associated in a fear network than can pictorial stimuli.

It can therefore be concluded from the two pictorial experiments that spatial segregation between primary and distracting information has to be minimized as much as possible. Although in spider phobia the ecological validity is much more evident for pictorial stimuli than for linguistic stimuli, larger attentional biases are found with the latter. It may bie that the attentional bias effects, found with linguistic stimuli, are somewhat inflated by post-attentional processing effects (see also MacLeod, 1991). In section 5.7 two possible explanations were proposed. The first explanation, that followed the Glaser and Glaser (1989) model and figure 5.7.2, suggested that the larger interference found with linguistic stimuli may be an output phenomenon. The second explanation, that words may result in more and polarized associations, suggested that interference effects may not be completely attributed to attentional processing, but also to anticipatory ruminations about the feared object or situation. As is stated in section 5.7, replication and dismantling of this issue will be of great theoretical interest.

\subsection{Adaptiveness of attentional bias: Activation, attention and behaviour}

As argued in section 3.8 , and as summarized in chapter 4 , it is hypothesized that selective attention towards threatening or otherwise meaningful stimuli could be adaptive in an organism. Attention to these stimuli may enable a subject to respond quickly and adequately. Generalizing this hypothesis, attentional bias will not be restricted to threatening stimuli, but will occur for any information that is of vital interest (either positively or negatively) and requires quick response. In this section, empirical evidence for the adaptiveness hypothesis itself will be discussed. In section 6.8 and onwards, the theoretical implications of the data are considered.

As argued in chapter 4, several specific hypotheses can be derived from the adaptiveness hypothesis. Firstly, it suggests that normal, nondisturbed subjects will show an attentional bias towards stimuli that require an urgent response. A second implication of the adaptiveness hypothesis is that an attentional bias towards specific stimuli must enhance facilitated response to these stimuli.

The experiments described in the sections 5.3 and 5.5 are relevant for the confirmation of the first hypothesis. In both studies two groups of normal subjects (students) were compared. One group was exposed to experimental manipulations, meant to create an urge to act; the other group was a nonmanipulated control group. In the section 5.3 experiment the induced urge in the experimental group was an approach response; the experimental group was instructed not to eat for 24 hours prior to the Stroop task, whereas the control group were instructed to eat that day as normal. Words from three different categories were presented to the two groups: food words, neutrall words and general positive words. The manipulation check demonstrated that 24 hours of fasting resulted in a positive evaluation of food, and an increased urge to obtain food. According to our hypothesis, the subjects in the fasting group were thus expected to show an attentional bias towards food words. The results of the section 5.3 experiment provided mixed evidence, depending on the control stimuli used. In section 5.3, two reasons for these slight effects were suggested. Firstly, it may be difficult to evoke a strong motivation to 
act immediately in nomal subjects. Secondly, it may be difficult to evoke such motivation for positively valenced stimuli. In other words, it may be difficult to make approach behaviour as vital as avoidance behaviour can be:

In the section 5.5 experiment, subjects in the experimental group were urged to avoid cognitively, i.e. they were instructed to avoid all thoughts about "numbers". In a former experiment, presented in section 5.4, it was demonstrated that subjects had great difficulty in trying to suppress a specific thought, although they tried hard to do so. In the section 5.5 experiment, the motivation to avoid all thoughts about "numbers" was further enhanced by suggesting to the subjects that we could monitor their mental processes. Both the experimental , and a nonmanipulated control group carried out a modified Stroop test that included number words and non-number contral words. It was hypothesized that a strong motivation to avoid all thoughts related to "numbers" would result in an attentional bias towards number words. Compared with the control group the thought suppression group indeed showed an attentional bias towards number words, thus supporting the hypothesis. In section 5.5, it was therefore demonstrated that an attentional bias towards for merly neutral stimuli can be induced in normal subjects, only by motivating them to avoid all thoughts about these stimuli. In section 5.5 , it was argued that subjects do not selectively attend to whatever subject is on their mind, and that the attentional bias effects towards number words, as found in this experiment, must be attributed to the motivation to avoid all the relevant thoughts. From the experiments described in sections 5.3 and 5.5 it appears that a strong motivation (an 'urge') to approach or avoid may result in an attentional bias towards stimuli representing the topic to be approached or avoided.

The second hypothesis derived from the adaptiveness model, i.e. that an attent ional bias towards specific stimuli must enhance facilitated response to these stimuli, is tested in the section 5.6 experiment. The flanker paradigm allowed for examination of both attentional bias and facilitated responding. In section 6.6 it was stated that in the flanker test, subjects had to concentrate on the central picture and had to ignore the flanking ones. It was hypothesized that (1) spider phobics would respond slower to nonthreatening central pictures which were flanked by pictures of spiders (see section 6.6) and that (2) spider phobics, as a consequence of this attentional bias, would show facilitated responses to slides with spiders presented as central pictures. That is, compared to slides with three nonthreatening pictures, spider phobics would respond more quickly to slides with a centrally presented spider flanked by two nonthreatening pictures. As argued already in section 6.6, attentional bias was difficult to demonstrate, probably because of spatial segregation. However, spatial segregation could not disturb the supposed facilltated responding to the centrally presented spiders. Untreated spider phobics indeed responded more quickly to centrally presented spiders than to centrally presented nonthreateri. ing animals. After treatment, they still tended to respond more quickly to centrally presented spiders, but the facilitated response was significantlly reduced. Thus, although in the section 5.6 experiment there was some difficulty in demonstrating attentional bias effects, the results confirmed the notion that phobic subjects would show facillitated responses to threatening stimuli and that these facilitated responses would be reduced after treatment.

If a causal relationship between a strong motivation to respond and selective attention to relevant stimuli existed, then a decrease in motivation would result in a decreased attentional bias effect. Two out of three experiments with spider phobics 
yielded support for this line of reasoning; a reduction of fear and avoidance motivation produced a reduction in attentional bias (see also section 6.2 for a discussion of the treatment effects). Moreover, in the experiment described in section 5.1 . spider phobics were treated with either an elaboration or a nonelaboration exposure in vivo treatment. The difference between the two exposure treatments was that in the elaboration treatment both motoric and cognitive avoidance were prevented, whereas in the nonelaboration treatment only motoric avoidance was prevented. The elaboration treatment was expected to be more effective in reducing anxiety, motivation for avoidance responses, and attentional bias. However "the elaboration and nonelaboration exposure in wivo treatment proved to have comparable treatment effects on anxiety and avoidance responses (see also Arntz \& Lavy, 1993a). and consequently, there was no evidence of a larger attentional bias reduction due to the elaboration instructions. Therefore, the elaboration data from the section 5.1 experiment could not provide additional evidence for the adaptiveness model.

To summarize the findings discussed, results of several experiments demonstrate that attentional bias can occur for stimuli that trigger an urge to act immediately (see the sections 5.1,5.3,5.5 and 5.7), and that this attentional bias decreases if responding is no longer urgent (see sections 5.1 and 5.7 ). Furthermore, anxious subjects show facilitated responses to threatening stimuli, but a direct association between attentional bias and facilitated response could not be demonstrated in the section 5.6 experiment.

\subsection{Attentional bias and cognitive theories}

As discussed in section 2.3, from the cognitive models of Beck (Beck \& Emery, 1985), Bower (1981, 1987) and Lang (1977, 1979) similar predictions can be made about selective processing of anxiety-relevant information. These theories suggest that the attentional bias shown by anxious subjects reflects complex cognitive structures that are specific to anxiety disorders. In section 3.4 , it is therefore argued that cognitive theories consider attentional bias as a relatively stable phenomenon, occurring in a specific subgroup of subjects. MacLeod and Rutherford (1992, p. 480), however, are of a different opinion and suggest that "According to Beck, selective processing will be associated with enduring trait variables, relating to anxiety vulnerability. In contrast, Bower proposes that this hypothetical processing bias, favouring threatening stimull, will be a cognitive correlate of an individual"s current level of state anxiety". This distinction between the models of Beck and Bower seems to be an artificial one. In contrast to Beck, who hypothesized that over-active danger schemata colour all input information, Bower did indeed suppose that a network has to become activated. MacLeod and Rutherford (1992) assumed that only state anxiety is able to activate a fear network, but this assumption is not plausible for two reasons. Firstly, it is not in line with the empirical data. MacLeod and Rutherford's (1992) suggestion would imply that anxious subjects have to be actually anxious whille they are performing a Stroop task. Although in some experiments the anxious subjects know they will be confronted with feared objects or situations after the Stroop task, in most attentional bias experiments (e.g. our section 5.2 experiment, but also the majority of experiments presented in table 3.1 ) the Stroop task is presented on its own. There seems, then, no reason for subjects particularly those with specific anxieties - to be in an anxious mood, and yet they 
show an attentional bias towards threatening words. Secondly, the essence of a network explanation is that several nodes and concepts are associated and that activation of one node results in a spreading of activation through the complete network. There is however no plausible basis for assuming that this spreading of activation has to start with the triggering of the emotional node within the network. In fact, Bower (1987, p.443) himself suggested that the activation of the emotional node can be secondary; by stating that "... while mentally reviewing the scenes of a funeral in vivid imagery, I am likely to reactivate and hence 'relive' a weakened version of the sadness I felt at the original event". Thus, a fear network can be trig gered not only by a current anxious mood, but also by associated nodes such as the stimuli presented in a Stroop task.

In section 6.2 the effects of a one- and two-session exposure in vivo treatment were discussed and it was demonstrated that attentional bias can even be significantly reduced, as was shown in section 5.1, in about two hours. As soon as current anxiety was reduced, the attentional bias towards threatening information was reduced also. In section 6.2 , it was argued that such strong reactivity of attentional bias effects is in conflict with the cognitive model of attentional bias. Although none of the cognitive theories is very exact with regard to how much time will be needed for cognitive structures to change, it is implicitly suggested that such change will take some time. For instance, Foa and Kozak (1986, p.28) stated that ".... short-term habituation sets the stage for changes in representations of threat regarding the fear responses...." and (p.29) ".... The proposed scenario maintains that within-sessions habituation can be relatively independent of higher order cognitive processes, whereas longer term habituation reflects changes in the patients' representations of threat."

Not only can selective attention effects be reduced in a few hours, the initiation does not have to take much time either. Studies on the behavioural function of attentional bias, discussed in section 6.7 , show that attentional biases can be rapid. Iy induced in normal subjects. For instance, in the section 5.5 experiment, a few minutes after the thought suppression instruction, normal subjects already showed an attentional bias towards number words. Of course it may be suggested that cognitive structures may be reduced in a few hours, and even that they might be developed in a few minutes, but it seems more prudent and plausible to suggest that attentional bias is relatively independent of enduring personality characteristics. such as cognitive fear structures, and has more to do with current concerns. As stated in section 3.3 however, it cannot be argued that subjects show an attentional bias towards whatever subject is on their mind (see for instance the studies by Mogg \& Marden, 1990; Wegner \& Erber, 1992). In the next section, our experimental data will therefore be integrated in a speculative model in which the motivation to respond will occupy a central place.

\subsection{A speculative integration of findings}

In section 3.8 , it was suggested that the adaptiveness hypothesis may be incor. porated into Lang's theory concerning fear structures and response programmes. By attending to a threatening stimulus, not only the stimulus nodes, but also the associated nodes of meaning ('danger!') and responding ('fly!') may become activated, and the subject may be able to escape as quicklly as possible. Atterition to other 
meaningful stimuli may activate other relevant response programmes and may consequently enable a subject to produce quick responses that are adequate for those particular stimuli.

The finding that an ensuing motivation to respond immediately creates an attentlonal bias (see sections 5.3 and 5.5 , discussed in section 6.7 ), suggests that there may be al causal rellationship between a strong motivation for acting in a certain way and attentional bias. But the finding that reduced motivation to avoid immediately alters the attentional bias (see sections 5.1 and 5.7 ) and the response performance (see section 5.6, discussed both in the sections 6.2 and 6.8 ) suggests that an interpretation of attentional bias in terms of enduring cognitive structures is superfluous. In other words, there is evidence for a causal relationship between the urge to respond and attentional bias to specific stimuli, but there is no evidence that enduring fear networks and complex response programmes are necessarily involved in attentional bias.

In figure 6.1 , a speculative interpretation is given of the potential behavioural function of attentional bias.

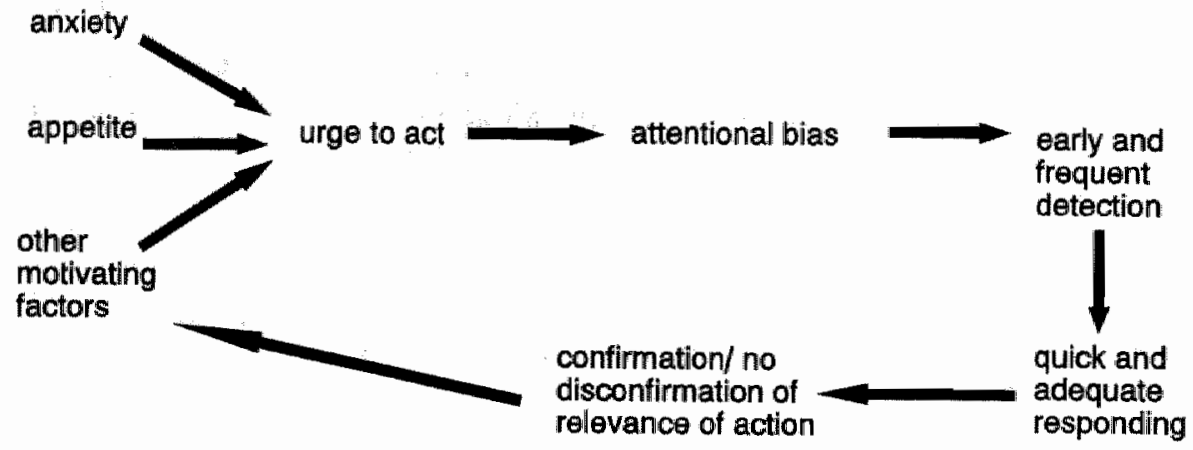

Figure 6.1: A speculative integration of findings

A strong motivation to respond to certain stimuli in a certain way may be the consequence of divergent experiences. Anxiety may be a very potent motivating factor, but it is certainly not the only one. The manipulation check in the section 5.5 experiment suggests that an explicit instruction to avoid all thoughts about a certain topic can also motivate subjects to respond as quickly as possible to relevant stimuli. And the rating scales data, presented in section 5.3, suggests that food deprivation can motivate normal subjects to approach food stimuli. There seem to be many ways, therefore, in which an urge to act can be induced.

Once such an urge is established, a subject is thought to be motivated to detect important stimuli as soon as possible and, in order to promote such early detection, a subject will selectively attend to all stimuli that may be relevant. As stated in the summary of section 6.7, results of several of our experiments demonstrate that 
attentional bias can occur for urgent stimuli. Results of other attentional bias experi. ments with normal subjects also suggest that a strong motivation to act may be causal to attentionall bias. In section 3.9 an experiment by Merckelbach et al. (1990b) was described, in which normal subjects were exposed to a classical conditioning experiment. They were aversively conditioned to a pictorial stimulus and afterwards showed an attentional bias towards this stimulus. And in addition to our section 5.3 experiment, a recent study by Overduin, Jansen and Louwerse (1993) showed that, after eating a small appetizer, normal subjects showed an attentional bias towards food words. In line with the model presented in this section, Overduin et al. (1993) concluded that craving for food results in selective attention to food stimuli in normal subjects.

The early detection of relevant stimuli enables the subject to respond immediately and adequately: rapid avoidance responses to threatening stimuli, and rapid approach responses to appetitive stimuli. In section 5.6 it was demonstrated that anxious subjects can indeed respond more quickly to threatening stimuli than to nonthreatening stimuli.

If the urge to act is realistic, selective attention towards relevant stimuli will indeed be adaptive and the need for quick response will be confirmed. But if this urge is not realistic, and consequently attentional bias is not adaptive, the urge to act quickly may not extinguish spontaneously. In the case of anxiety disorders for instance, the urge to avoid certain stimuli is (per definition) exaggerated. But the efficient avoidance responses do not allow disconfirmation of the irrational fear to take place. The urge to react in this way to relevant stimuli, and consequently the attentional bias towards these stimuli, may therefore be maintained. Attentional bias appears to be a relatively stable effect, as long as the subject remains anxious (see Martin et al., 1992). But if quick response is prevented and the motivation to respond is disconfirmed by exposure in vivo treatment, the attentional bias will be immediately reduced (see also section 6.8).

\subsection{Critical remarks about the model: Theoretical considerations}

The model, as proposed in the previous section, leaves several issues to be discussed. Firstly, it is questionable whether various types of responses cian be grouped together, without differentiating between behavioural and cognitive responses, or approach and avoidance responses. To begin with the latter pair, the difference between approach and avoidance responses may indeed not be important. It is already known from operant conditioning procedures that both the presentation of appetitive stimuli and the termination of aversive stimuli result in an increase in response probability. Only the direction of response behaviour will be different: in the case of appetitive stimuli, approach behaviour will increase, whereas in the case of aversive stimuli, avoidance behaviour will increase (for a clear synopsis of operant reinforcing procedures see Gray, 1982, p.7). Results of several attentional bias studies (see section 5.3, and also Overduin et al., 1993) also suggest that, although a strong motivation for avoidance behaviour may be easier to accomplish, there is no fundamental difference between approach and avoidance behaviour.

The question to what extent cognitive and behavioural responses are comparable may be more complicated. Cognitive responses are less well defined than behaviou- 
ral responses, and it is sometimes unclear whether a specific covert process should be considered as a cognitive response or not. In section 5.5 for instance, some examples of very divergent covert processes are given, all of which are understood under the term "cognitive avoidance". In section 5.5, it is argued that these processes can be divided into automatic and strategic cognitive avoidance and that strategic cognitive avoidance seems to be highly characteristic of anxiety disorders. These strategic cognitive avolidance responses, such as deliberate suppression and distraction strategies, are carried out by a subject in order to avoid or actively escape a threatening stimulus. Strategic cognitive avoidance responses to threatening stimuli cian be considered as 'cognitive actions" , and they seem to be very similar to behavioural responses. Whether automatic cognitive avoidance responses, like repression and dissociation, are also comparable to behavioural responses is ques" tionable. The occurrences of these cognitive processes are not thought to be under conscious control and it is difficult to compare such unconscious cognitive processes with deliberate actions, such as strategic cognitive and behavioural avoidance responses. Since unconscious cognitive avoidance is difficult to investigate experimentally, the question as to whether it can be compared with deliberate avoidance responses is left unanswered for the this moment.

According to the figure 6:1 model, the urge to act is essential as to attentional bias effects. And although approach and avoidance responses cq. strategic cognitive and behavioural responses, may have different consequences, they all have a maintaining effect on the motivation to respond this way. Consequently, attentional bias effects will be sustained by urgent deliberate response, whatever the exact form of thils response.

A second issue is what process attentional bias reflects, if not enduring cognitive structures. According to the model, subjects show an attentional bias towards stimuli that require a quick response. From this point of view, attentional bias is seen as reflecting action tendencies of (groups of) subjects at a specific moment. Although these tendencies can remain stable for a long time, attentional bias effects will immediately adapt to changes in the urgency to act. In section 2.1, a distinction is made between the information processing and the functional ecological approach in cognitive psychology (see also Safran \& Greenberg, 1988). Although the concept of attentional bias is, by definition, derived from information processing theories, it is obvious that the model proposed in the previous section approaches attentional bias as a functional ecological phenomenon.

Emotion theorists like Frijda (1986), and Greenberg and Safran (1987) have previously suggested that emotions reflect action tendencies. For instance, Greenberg and Safran (1987) proposed a model in which emotions, cognitions and behaviour are highly integrated. They stated that: "...... The type of emotion system that we have described has the advantage of rapidly activating adaptive behavior in response to an event that requires action. Thus, there is an immediate, rapid perceptual appraisal of situations that gives rise to physiological and expressivemotor responses that are action tendencies in the worll. Human beings are adaptive organisms constantly adjusting themselves to and attempting to master the environment..." 1 Greenberg \& Safran, 1987, p.3031. If the data from the chapter 5 experiments and the section 6.9 model warrant further theoretical foundation, integration into such a functional ecological framework would seem fruitful.

A last question is whether other psychopathology-related biases in information processing are relatively independent of enduring cognitive structures. In chapter 2 , 
it was argued that the experimental data concerning identification, and even more so, the data concerning retrieval of threatening information are difficult to explain using traditional cognitive theories of anxiety disorders. It would be interesting to investigate whether biases in these later stages of information processing do not reflect enduring cognitive structures as well.

\subsection{Critical remarks about the model: Clinical implications}

It has to be noted first that little is known about the clinical applicability of attentional bias and that it has not been a subject of investigation in this dissertation either. Therefore, suggestions about the clinical use of attentional bias measures are tentative.

It is sometimes suggested that attentional bias may be of diagnostic significance in anxiety disorders. In section 5.1, it was shown that the correlations between Stroop deterioration and two anxiety measurements, behavioural approach and selfreported fear, are weak. In other studies correlations between measurements of attentional bias and anxiety are also weak, although sometimes significant $(\mathrm{e.g}$. Hope et al., 1990; MacLeod \& Hagan, 1992; Mathews \& MacLeod, 1985). Such imperfect correlations are to be expected in the light of the model presented in section 6.9. Whereas measurements of attentional bias are thought to reflect the urge to avoid the threatening stimuli, behavioural approach tests show the net effect of this urge and the opposite urge, i.e. the 'will-power' to approach the threatening stimulus. And ratings scales reflect quite a different aspect of fear, namely the subjective experience of someone's emotional state. Results of the section 5.1 experiment do indeed suggest that a reduction in attentional bias correlates more highly to an improvement in approach behaviour than to a decrease in self-reported fear.

Can attentional bias measures give additional information in the diagnostic process? Attentional bias may be preferable to self-reports as a cognitive measure. Attentional bias measures are less susceptible to demand effects and other methodological weaknesses that are associated with self-report data isee also section 2.2). The question is, exactly what cognitive processes are measured by Stroop tasks and other attentional bias tests. As argued in the previous sections, our data does not support the claim that attentional bias reflects complex and enduring cognitive structures. But we did find some evidence for the suggestion that subjects selectively attend to stimuli that require a rapid response. Thus, attentional bias measures may give more information about the particular stimuli an anxious subject is strongly motivated to avoid.

As argued in section 5.1, there appears to be no practical need for the measurement of attentional bias in most clinical cases. But there might be circumstances in which additional information is warranted, for example when a highly traumatized subject denies being anxious. Although there is some evidence to suggest that attentional bias at group level may differentiate between traumatized subjects with and without post-traumatic stress disorder (Foa et al., 1991; McNally et al., 1990a), the extent to which attentional bias measures are meaningful in individual cases remains unclear.

In section 5.1, it was suggested that attentional bias mav be valuable as a criterium for successful treatment or as a prognostic factor for relapse after treat- 
ment. To the degree that measurement of attentional bias reflects an urge to avoid fear-related stimul, these may be useful clinical applications.

But, as stated above, attentional bias has never been used as a test for the existence of anxiety disorders or other emotional states in an individual. And at the present it is unclear whether attentional bias can be used to that end.

\subsection{Concluding remarks: Recommendations for future research}

Various issues related to the concept of attentional bias and its significance have been dealt with in this dissertation. The experimental studies in chapter 5 supported some earlier findings reviewed in chapter 3 , and may have provided an answer to some questions raised in chapters 3 and 4 . But other issues, especially those related to the behavioural function of attentional bias, require more experimental research. Because of the potential theoretical and clinical relevance of the model presented in section 6.9 , future research should concentrate on these issues. Replications of the attentional bias effects towards appetitive stimuli are particularly recommended. Additional experimental research into the relationship between attentional bias and the capability to act immediately is also warranted. New investigations of simultaneously measured attentional bias and facilitated performance would therefore be welcomed.

Furthermore, as stated in section 6.6 , the question as to whether performance is affected by post-attentional processes in linguistic Stroop tests needs clarification; settling this matter may lead to a reconsideration of the use of linguistic and pictorial attentional bias tasks.

When the above-mentioned issues have been clarified, research on clinical issues may be warranted. It would be useful to investigate whether attentional bias can be used as a diagnostic or prognostic factor in anxiety disorders. In four studies we used anxious subjects that met the DSM-III-R criteria for simple phobia (three studies) and obsessive compulsive disorder (one study). Although at group level the anxious subjects selectively attended to threatening stimuli, in each study there were numerous anxious subjects who did not show such a bias. In order to examine the potential relevance of attentional bias measures as a diagnostic or prognostic factor, clinical investigations will first have to concentrate on the question whether. at group level, anxious subjects with and without attentional bias differ with respect to e.g. seriousness of subjective anxiety and avoidance behaviour, treatment effects and relapse rates. If they do indeed differ, it might be worthwhile considering the use of attentional bias measures at an individual level. Because of the large interindividual differences, difficulties in determining specific standards for clinical attentional bias and improvement can, however, be expected. It will be a long time, then, before attentional bias measures are of use in clinical practice as a more or less objective test for the processing of threatening information. In fact it is doubtful whether attentional bias will ever be used to that end.

Attentional bias measures may be useful in a quite different, and perhaps more controversial, field of research. In anxious subjects, the deterioration in colour naming threatening words is only a few dozen milliseconds and will be difficult to simulate. Using a modified Stroop task, it may be possible to differentiate between subjects that are actually anxious and subjects that pretend to be. In order to use modified Stroop tasks as "lie detector", subjects should be informed beforehand that 
anxious subjects are unwittingly slow in collour naming the threatening words. When they are instructed to colour name as quickly as possible, it would be expected that anxious subjects would show a small or no attentional bias, whereas shammers would show an improbably large attentional bias.

This all may sound very promising, but it has to be realized that, before attentional bias can be applied into clinical practice, either as a diagnostic cq. prognostic factor or as a "lie detector", a great deal of fundamental research will have to be carried out. 


\section{Samenvatting}

Sinds een tiental jaren ïs de wijze waarop angstige mensen hun aandacht verdelen een geliefd anderwerp voor experimentele studies. In dit proefschrift wordt verslag gedaan van een aantal van dergelijke experimenten, uitgevoerd door de auteur en diverse co-auteurs. Vier theoretische hoofdstukken gaan vooraf aan deze empirische verslaglegging. Deze inleidende hoofdstukken hebben een trechtervormige opbouw, van zeer algemeen (hoofdstuk 1) tot specifiek gericht op de onderzoeksvragen die in dit proefschrift aan bod komen (hoofdstuk 4). leder hoofdstuk wordt voorafgegaan door een samenvatting van hetgeen gepresenteerd wordt, zodat hier volstaan zall worden met een kort overzicht.

In hoofdstuk 1 wordt in vogelvlucht de opkomst van de leertheorieen en de gedragstherapie behandeld. Besproken wordt hoe, na een aanvankelijke jubelstemming, alras kritiek werd geuit op enkele aannames van de klassieke conditioneringstheorie van angst. Voorts hoe deze conceptuele kritiek resulteerde in een omschakeling in het leertheoretisch denken wan reflexologisch naar cognitief leren.

In hoofdstuk 2 worden de cognitieve theorieën van respektievelijk Beck, Bower en Lang gepresenteerd. Zelfrapportagle studies lijken deze theorieen te ondersteunen, maar hebben ernstige methodologische tekortkomingen. Daarom concentreer ik mij op een experimentele toetsing van cognitieve aannames. De drie besproken cognitive theorieën veronderstellen alle dat angstige mensen prioriteit geven aan informatie die in overeenstemming is met hun gemoedsgesteldheid. In dit hoofdstuk wordt dieper ingegaan op de resultaten van experimentele studies naar de veronderstelde neiging van angstigen om angstrelevante informatie seliektief te identificeren, te interpreteren en te herinneren.

Een apart hoofdstuk wordt gewijd aan de neiging selektief aandacht te besteden aan potentieel bedreigende informatie "de "attentional bias". In hoofdstuk 3 worden experimentele studies naar deze aandachtsverstoring bij angstige mensen bespro. ken. De resultaten van deze experimenten steunen in het algemeen de hypothese dat angstigen, en onder bijzondere omstandigheden wellicht ook niet-angstigen, selektief aandacht besteden aan bedreigende informatie. Een zestal meer of minder onderzochte kwesties aangaande deze aandachtsverstoring wordt uitgediept.

Hoofdstuk 4 geeft een overzicht van de onderwerpen en voornaamste hypotheses van de experimentele studies, welke gepresenteerd worden in hoofdstuk 5 .

In hoofdistuk 5 wordt verslag gedaan van zeven experimenten. De paragrafen 5.1 tot en met 5.6 zijn in de huidige vorm reeds gepubliceerd danwel geaccepteerd voor publikatie. Paragraaf 5.7 komt grotendeels overeen met een artikel in druk, maar bevat een theoretisch meer diepgaande diskussie. Kort samengevat zijn de belangrijkste bevindingen uit deze experimenten:

1 Wanneer gebruik wordt gemaakt van traditionele linguistische aandachtstests, worden de selektieve aandachtseffekten gerepliceerd bij spin fobici en obsessief-compulsieve patiënten. 
Reduktie van de angst leidt tot een onmiddellike daling van deze selektieve aandachtseffekten. Een dergelijke reaktiviteit is in strijd met de hypothese dat deze aandachtsverstoring en weerspiegeling is van langdurende persoanlijkheidstrekken.

3 De selektieve aandachtseffekten kunnen niet verklaard worden door een grotere bekendheid van angstige mensen met angstrelevante informatie.

De selektieve aandachtseffekten kunnen niet verklaard worden door een sterkere emotionele waarde van angstrelevante stimuli.

5 Er is geen bewijs gevonden voor de stelling dat vermijding de reden is waar. om er geen selektieve herinneringseffekten worden gevonden bij angstigen.

6 Op een pictorale aandachtstaak vertonen spin fobici kleinere selektieve aandachtseffekten dan op een linguistische aandachtstaak. Dit is in strijd met onze hypothese dat de grotere ecologische validiteit van een dergelijke pictorale taak gepaard zal gaan met een sterker selektief aandachtseffekt.

Hongerige normale proefpersonen lijken selektief aandacht te besteden aan voedsel stimuli. En wanneer normale proefpersonen glevraagd wordt een gedachte te onderdrukken, dan besteden zij selektief aandacht aan stimuli die gerelateerd zijn aan deze gedachte. Beide bevindingen suggereren dat mensen selektief aandacht schenken aan stimuli die een snelle respons (toenadering of vermijding) vereisen.

8 Spin fobici reageren sneller op plaatjes van spinnen dan op niet-bedreigende dierplaatjes. Dit zou in overeenstemming zijn met de hypothese dat angstigen selektief alandacht besteden aan bedreigende informatie, teneinde snel te kunnen reageren.

Hoofdstuk 6 is gewijd aan een algemene bespreking en integratie van deze bevindingen. Een speculatief model aangaande de mogelijke gedragsmatige funktie van selektieve aandacht wordt voorgesteld. Centraal in dit model staat de drang om aktie te ondernemen. Deze drang kan zijn oorsprong vinden in angst, maar ook in andere sterk motiverende faktoren. In het model wordt gesuggereerd dat selektieve aandacht het gevolg is van een dergelijke drang tot handelen. Relevante stimuli zullen snel worden gedetekteerd, waardoor de betreffende persoon snel in staat is aktie te ondernemen. De motivatie om op een dergelijke wijze te reageren, en daarmoe ook de aandachtsverstoring, zal hiermee in stand gehouden worden. 


\section{Summary}

Since around ten years, the manner how anxious subjects divide their attention is a popular subject of experimental studies. A couple of these experiments, executed by the author and several co-authors, are described in this dissertation. Four theoretical chapters precede these empirical reports. These introductory chapters have a funnel-shaped construction, from very general (chapter 1) to specifically aimed at the topics of research as presented in this dissertation (chapter 4). Each chapter will be preceded by a summary of what is presented, as such this summary is confined to a short overview.

Chapter 1 shows the origins of the learning theories and behaviour therapy in a bird-eye's view. The discussion contains that, after a period of jubilation, criticisms were raised on some of the assumptions of the classical conditioning theory of anxiety. And how these conceptual criticisms resulted in a change in thought of the learning theories from reflexive to cognitive learning.

In chapter 2 the cognitive theories of Beck, Bower and Lang respectivelly are presented. Self-report studies seem to support these theories, but have serious methodological shortcomings. Therefore I concentrate myself on an experimental investigation of the cognitive assumptions. The three discussed cognitive theories all suppose that anxious subjects give priority to mood-congruent information. This chapter goes further into the results of experimental studies concerning the hypoth esized inclination of anxious subjects to selectively identify, interpret and remember anxiety-relevant information.

A separate chapter is dedicated to tendency to pay selective attention to potentilally threatening information, the so-called "attentional bias". Chapter 3 presents the experimental studies of attentional bias in anxious subjects. The results of these experiments confirm in general the hypothesis that anxious subjects and possibly, under certain circumstances, also nonanxious subjects, selectively attend to threatening information. Six, more or less researched, issues concerning attentional bias are discussed in more detail.

Chapter 4 is an overview of topics and main hypotheses of the experimental studies, which are presented in chapter 5.

In chapter 5 seven experiments are reported. Sections 5.1 up until 5.6 are already published or accepted for publication in their present form. Section 5.7 mostly compares with an article in press, but contains a more profound theoretical discussion. The more important findings of these experimental studies are summarized as follows:

1 When traditional linguistic attentional bias tests are used, the selective attention effects are replicated in spider phobics and obsessive compulsive patients.

2 Reduction of anxiety results in an immediate decrease of the selective attention effects. Such a reactivity is in conflict with the hypothesis that attentional bias reflects enduring personality traits. 
3 The selective attention effects cannot be explained by greater familiarity of anxious subjects with anxiety-relevant information.

4 The selective attention effects cannot be explained by stronger emotional valences of anxiety-relevant stimuli.

5 No evidence is found for the hypothesis that avoidance strategies can account for the lack of selective memory effects in anxious subjects.

6 In spider phobics smaller attentional biases are found with pictorial attentional bias tests rather than with linguistic attentional bias tests. This is in conflict with the hypothesis that the greater ecological validity of such a pictorial task will be associated with a more prominent attentional bias effect.

7 Hungry normal subjects seem to attend selectively to food stimuli. And as a result of a thought suppression instruction, normal subjects selectively attend to stimuli related to that thought. Both findings suggest that subjects selectively attend to stimuli that require a quick (approach or avoidance) response.

8. Spider phobics can respond more quickly to pictures of spiders than to pictures of nonthreatening animals. This concurs with the hypothesis that an attentional bias towards threatening information allows for quick responding.

Chapter 6 is dedicated to a general discussion and integration of these findings. A speculative model concerning the potential behavioural function of selective attention is postulated. The urge to act takes a central place in this model. This urge can stem from anxiety or other strongly motivating factors. The model suggests that selective attention effects result from such urges to act. Relevant stimuli are quickly detected by which the subject in question can respond quickly. The motivation to respond in this way, and therefore the attentional bias, will thus be maintained. 


\section{References}

Achille, M., Stewart, S.H., Dubcis-Nguyen, I., Peterson, J.B., \& Pihl, R.0. 11991). Social fear and selective attention bias for non-verbal social threat. Paper presented af the Canadian Psychological Association Annual Meeting. Calgary, June 1991.

Agras, S. Sylvester, D., \& Oliveau, D. (1969). The epidemiology of common fears and phobias. Comprehensive Psychiatry, 10, 151-156.

Allen, B.P. \& Potkay, C.R. 119811 . On the arbitrary distinction between states and traits. Journal of Personality and Social Psychology, 41, 916-928.

American Psychiatric Association (1987). Diagnostic and Statistical Manual of Mental Disorders (3rd edition, revised). Washington, $D C$.

Arntz, A. \& Lavy, E. (1993a). Does stimulus elaboration potentiate exposure in vivo treatment? Two forms of one-session treatment of spider-phobia. Behavioural Psychotherapy, 21, 1-12.

Arntz, A., Lavy, E., Berg, G. van den, \& Rijsoort, S. van (1993b). Negative beliefs of spider phobics: A psychometric evaluation of the Spider Phobia Bellefs Questionnaire. Advances in Behaviour Research and Therapy, 15, 257-277.

Bandura, A. (1969). Principles of behavior modification. Holt, Rinehart and Winston, New York.

Bandura, A. (1977). Self-efficacy: Toward a unifying theory of behavitoral change. Psychological Review, 84, 191-215.

Beck, A.T. (1976). Cognitive Therapy and the Emotional Disorders. International Universities Press, New York.

Beck, A.T. \& Emery, G. (1985). Anxiety Disorders and Phobias: A Cognitive Perspective. Basic Books Inc., New York.

Beck, A.T., Laude, $R_{*}$ \& Bohnert, M. (1974), Ideational components of anxiety. Archives af General Psychiatry, 31, 319-325.

Beck, A.T. \& Rush, A.J. (1975). A cognitive model of anxiety formation and anxiety resolution. In: I. Sarason, \& C. Spiellberger (Eds.). Stress and Anxiety (vol. 2). John Wiley and Sons, New York.

Beck, J.G., Stanley, M.A., Averill, P.M., Baldwin, L.E., \& Deagle E.A. (1992). Attention and memory for threat in panic disorder. Behaviour Research and Therapy, 30, 619-629.

Bentall, R.P. \& Thompson, M. (1990). Emotional Stroop performance and the manic defence. British Journal of Clinical Psychology, 29, 235-237.

Ben-Tovim, D.I. \& Walker, M.K. (1991). Further evidence for the Stroop test as a quantitative mealsure of psychopathology in eating disorders. International Journal of Eating Disarders, 10,609 . 613.

Ber-Tovim, D.I. Walker, M.K., Fok, D., \& Yap, E. (1989). An adaptation of the Stroop test for measuring shape and food concerns in eating disorders: A quantitative measure of psychopathollogy? International Journal of Eating Disorders, $8,681-687$.

Blaney, P.H. (1986). Affect and memory: A review. Psycholagical Bulletin, 99, 229-246.

Borkovec. T.D. \& Sides, J.K.11979). The contribution of relaxation and expectancy to fear reduction via graded imaginal exposure to feared stimuli. Behaviour Research and Therapy, 17, 529 540 .

Bouman, T.K., Scholing, A., Emmelkamp, P.M.G., \& Dijkstra, W. 11987). Anxiety Disorders Interview Schedule (ADIS) (intern report). Groningen: Univarsity of Groningen, Department of Psychology.

Bower, G.H. (1981). Mood and memory. American Psychologist, 36, 1129-148.

Bower, G.H. (1987). Commentary on mood and memory. Behaviour Research and Therapv, 25.443455.

Bradley, M.M. (1993). Emotional memory: A dimensional analysis. In: S.H.M. van Gioozen, N.E. van den Poll, \& J.E. Sergeant (Eds). Emotions: Essays on emotion theary. Lawrence Erlbaum Assoc. Hillsdlale, New Jersey. 
Broadbent, D. \& Broddbent, M. (1988). Anxiety and attentional bias: state and trait. Cognition and Emotion, 2, 165-183.

Burgess, I.S, Jones, L.M., Riobertson, S.A., Radcliffe, W.N. \& Emerson, E. (1981). The degree of control exerted by phobic and non-phobic verbal stimuli over the recognition behaviour of phabic and nonphobic subjects. Behaviour Research and Therapy, 19, 233-243.

Butler, G. \& Mathews, A. (1983). Cognitive processes in anxiety. Advances in Behaviour Research and Therapy, 5, 51-62.

Cassidy, K.L., McNally, R.J., \& Zeitlin, S.B. (1992). Cognitive processing of trauma cues in rape victims with post-traumatic stress disorder. Cognitive Therapy and Research, 16, 283-295.

Channon, S. \& Hayward, A. (1990). The effect of short-term fasting on processing of food cues in normal subjects. International Journal of Eating Disorders, $9,447-452$;

Channon, S. Hemsley, D., \& De Silva, P. (1988). Selective processing of food words in anorexia nervosa. British Journal of Clinical Psychology, 27, 259-260.

Cloitre, M. \& Liebowitz, M.R. (1991). Memory bias in panic disorder: An investigation of the cognitive avoidance hypothesis. Cognitive Therapy and Research, 15, 371-386.

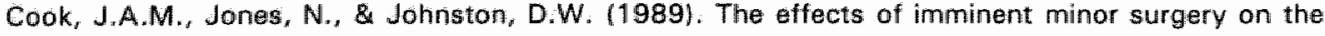
cognitive processing of health and interpersonal threat words. British Journal of Clinical Psychologr, $28,281-282$.

Cook, M. Mineka, S. Wolkenstein, B., \& Latsch, K. (1985). Observational conditioning of snake fear in unrelated thesus monkeys. Journal of Abnormal Psychology, 94, 591-610.

Cooper, M., Anastasiades, P., \& Fairburn, C.G. (1992). Selective processing of eating-, shape-, and weight-related words in persons with bulimia nervosa. Journal of Abnormal Psychology, 101, $352-355$.

Corteen, R.S. \& Wood, B. (1972). Automatic response to shock-associated words in an unattended. Jowrnal of Experimental Psychology, 94, 303-318.

Craske, M.G., Street, L.L., Jayaraman, J., \& Barlow. D.H. 11991\}. Attention versus distraction during in vivo exposure: Snake and spider phobias. Journal of Anxiety Disorders, 5, 199-211.

Dalgleish, T. \& Watts, F.N. (1990). Biases of attention and memory in disorders of anxiety and depression. Clinical Psychology Review, 10, 589-604.

Davey, G.C.L. (1989a). Dental phobias and anxieties: Evidence for conditioning processes in the acquisition and modulation of learned fear. Behaviour Research and Therapy, 27, 51-58.

Davey, G.C.L. (1989b). UCS revaluation and conditioning models of acquired fears. Behaviour Research and Therapy, 27, 521-528.

Davis, P.J. \& Schwartz, G.E. (1987). Repression and the inaccessibility of affective memories. Journal of Personality and Social Psycholagy, 52, 155-162.

Dawkins, K. \& Furnham, A. (1989). The colour naming of emotional words. British Journal of Psychology, 80, 383-389.

De Houwer, J. \& Hermans, D. 17992a). Differences in the affective processing of words and pictures. Submitted for publication.

De Houwer, $d$. and d'Ydewalle, G. (1992b). Evidence against the Glaser and Glaser (1989) model of the Stroop effect. Submitted for publication.

Dickinson, A. (1987). Animal conditioning and learning theory. In : H.J. Eysenck, \& I. Martin (Eds.). Theoretical foundations of behavior therapy. Plenum Press, New York.

Easterbrook, J.A. (1959). The effect of emotion on cue utilisation and the organisation of behavior. Psychological Review, 66, 183-201.

Edwards, S. \& Dickarson, M. (1987). On the similarity of positive and negative intrusions. Behaviour Research and Therapy, 25, 207-211.

Elhlers, A., Margraf, J., Davies, S. \& Roth, W.T. (1988). Selective processing of threat cues in subjects with panic attacks. Cognition and Emotion, 2, 201-219.

Eifert, G.H. (1987). Language conditioning, clinical issues and applications in behavior therapy. In: H.J. Eysenck, \& 1. Martin (Eds.). Theoretical foundations of behavior therapy. Plenum Press, New York.

Emmelkamp. P.M.G. (1975). Effects of expectancy on systematic desensitization and flooding. Joumal of Behavioral Analysis and Modification, 1, 1-11.

Emmelkamp. P.M.G (1982). Phobic and Obsessive-Compulsive Disorders - theory, research and practice. Plenum Press, New York. 
Emmelkamp, P.M.G. (1991). Treatment of obsessive-compulsive disorden" From standardized to broad-spiectrum cognitive behavior. Paper presented at the Internatonal Congress of Stress; Anxiety and Emotional disorders. Brage, Portugal.

Eysenck, H.J. (1968). A theory of the incubation of anxiety/fear responses. Behaviour Research and Therapy, 6, 319-321.

Eysenck, H.J. (1976). The learning theory model of neurosis: a new approach. Behavour Research and Therapy, 14, 251-267.

Eysenck, H.J. (1987). Behavior therapy. In: H.J. Eysenck, \&. Martin IEds.). Theoretical foundations of behawior therapy. Plenum Press, New York.

Evsenck. M.W. (1982). Attention and Arousal: Cognition and Performance. Springer-Verlag, Berlin.

Exsencik, M.W. (1984). A handbook of Cognitive Psychology. Erlbaum Press, London.

Eysenck, H.d. \& Eysenck, M.W. (1985). Personality and individual differences. Plenum Press, Niew York.

Eysenck, M.W. (1988). Anxiety and attention. Anxiety Research, 1, 9-15.

Eysenck, M.W., Macleod, C., \& Mathews, A. 11987a). Cognitive functioning and anxhety. Psychological Research, 49-189.195.

Eysenck, M.W. \& Mathews, A. (1987b). Trait anxiety and cognition. In: H.J. Eysenck, \& I. Martin (Eds.). Theoretical foundations of behavior therapy. Plenum Press, New York.

Eysenck, M.W., Mogg, K., May, J., Richards, A., \& Mathews, A. (1991). Bias in the interpretation of ambiguous sentences related to threat in anxiety. Journal of Abnormal Psychology, 100, 144 150 .

Fairburn, C.G., Cooper P.J., Cooper, M.J., McKenna, F., \& Anastasiades, $P_{n}$ (1991). Salective infor" mation processing in bulimia nervosa. International Journal of Eating Disorders, 10, 415-422.

Foa, E.B. \& Emmelkamp, P.M.G. (1983). Failures in behavior therapy. John Wiley and Sons, New York.

Foa, E.B., Feske, U. Murdock, T.B., Kozak, M.J., \& McCarthy, P.R. 11991). Processing of threatrellated information in rape victims. Journal of Abnormal Psychology, 100, 156-162.

Foa, E.B., Ilai, D., McCarthy, P.R., Shover, B., \& Murdock, T. (1993). Information processing in obsessive-compulsive disorder: Cognitive Therapy and Research, 17, 173-189.

Foa, E.B. \& Kozak, M.J. (1986). Emotional processing of fear: Exposure to corrective information. Psychological Bulletin, 99, 20-35.

Foa, E.B. \& Kozak, M.J. 11992). Pathological anxiety: Meaning and the structure of fear. In: N. Birbaumer, \& A. Öhman (Eds.). The Organization of Emotions. Hogrefe Int., Toronto.

Foa, E.B. \& McNally, R.J. (1986). Sensitivity to feared stimull in obsessive-compulsives: A dichotic listening analysis. Cognitive Therapy and Research, 10, 477-485.

Foa, E.B., McNally, R., \& Murdock, T.B. (1989a). Anxious mood and memory. Behaviour Research and Therapy, 27, 141-147.

Foa, E.B., Steketee, G., \& Rothbaum, B. (1989b). Behavioural/cognitive conceptualizations of post traumatic stress disorder. Behavior Therapy, 20, $155-176$.

Fredrikson, M. (1983). Raliability and validity of some specific foar questionnaires. Scamdinavian Journal of Psychology, 24, 331-334.

Fridhandler, B.M. (1986). Conceptual note on state, trait, and the state-trait distinction. Journal of Personality and Social Psychology, 50, 169-174.

Frijda, N.H. (1986). The Emotions. Cambridge University Press, Cambridge.

Frijda, N.H., Kuipers, P. \& Schure, E.ter (1989). Relations among temotion, appraisal, and emotional action readiness. Journal of Personality and Social Psychology, 57, 21 2-228.

Gerrig, R. \& Bower, G.H. 119821. Emotional influences on word recogmition. Bulletin Psychonomic Society, 19, 197-200.

Giles, M. \& Cairns, E. (1989). Colour naming of violencerelated words in Northern Ireland. British Journal of Clinical Psychology, 28, 87-8B.

Glaser, W.R. \& Düngelhoff, F.J. (1984). The time course of picture-word interferemce. Journal of Experimental Psychology: Human Perception and Perfarmance, 10, 640-654.

Glaser, W.R. \& Glaser, M.O. (1989). Context effects in Stroopmlike word and picture processing. Journal of Experimental Psychology: General, $118,13-42$.

Goldfried, M. (1971). Systematic desensitization as training in self-contirol, Journai of Consulting and Clinical Psychology, 37, 228-234. 


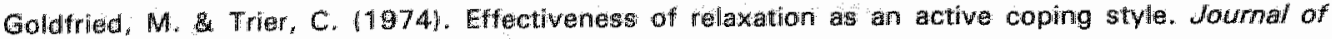
Abnomal Psychology; $3,348-355$.

Gothb, 1.H. \& McCann, C.D. (1984). Construct accessibility and depression: An examination of cougnitive and alfective factors. Journal of Personality and Social Psychology, 47, 427-439.

Gotlib, 1.H., McLachlan, A.L., \& Katz, A.N. (1988). Biases in visual attention in depressed and nondepiressad individuals. Cognition and Emotion, $2,185-200$.

Gray, J.A. (1982). The Newropsychology of Anxiety. Oxford University Press, New York.

Growberg, L.S. \& Safran, J. (1987). Entation, cognition, and action. lin. H.J. Eysenck, \& I. Martin (Edw.). Theoretical foundations of behavior therapy. Plenum Press, New Yorlk.

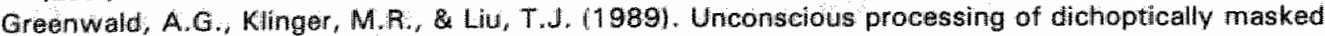
words, Memory and Cognition, 17, 35-47.

Hare, R.D. (1973). Orienting and defensive responses to visual stimuli. Psychophysiologv, 10, 453464.

Hekmat. H. (1972). The role of imagination in semantic desenisitization. Behavior Therapy, 3, 223231.

Hekmat, H. (1977). Semantic behawior therapy: Unidimensional or multidimensional. Behawior Therapy, 8, 805-809.

Hibbert, G.A. (1984). Ideational components of anxiety: Their origin and content. British Journal of Pisychiatry, 144, 618-624.

Hill, $A_{2} B_{n}$ \& Kemp-Wheller, S.M. (1989a). The inflluence of context on lexical decision times for emationally aversiwe words. Current Pswchology, 219-227.

Hill, A.B. Kemp-Wheeler, S.M. (1989b). The influence of anxiety on lexical and affective decision time for emotional words. Personality and Indivialual Differences, 10, 1143-1149.

Hope, D.A., Rapee, R.M. Heimberg. R.G., \& Dombeck, M.d. (1990). Representations of the self in social phobia: Vulnerability to social threat. Cognitive Therapy and Research, 14, \$77-189.

Hout, M. van den \& Merckelbach. H. (1991). Classical conditioning: Still going strong. Behavioural Psychotherapy, 19, 59-79.

Hugholahl, K. (1989). Simple phobias. In: G. Turpin (Ed.). Handbook of Clinical Psychophysiology. Wiley and Sons, Chichester.

Ingram, R.E., Kendall, P.C., Smith, T.W., Donnell, C., \& Ronan, K. 11987). Cognitive specificity in emotional distress. Journal of Persanality and Social Psychology, 53, 734-742.

Jacobson, E. (1938). Progressive relaxation. University of Chicago Press, Chicago.

Jones, M.C. (11924). The elimination of children's fears. Journal of Experimental Psychology, 7, 382390.

Kamin, L.J. (1969). Predictability, surprise, attention and conditioning. In: B.A. Campbell, \& R.M. Church (Eds.). Punishment and aversive behawiors. Appleton-Century-Crofts, New York.

Kaspi, S. \& McNally, R. (1991). Selective processing of ideographic emotional information in PTSD. Paper presented at the meeting of the Association for Advancement of Behavior Therapy, New York.

Kemp-Wheeler, S.M. Hill, A.B. (1987). Anxiety responses to subliminal experinece of mild stress. British Journal of Psychology, 78, 365-374.

Kamp-Wheser, S.M. \& Hill, A.B. (1992). Semantic and emotional priming below objective detection threshold. Cognition and Emation, 6, 113-128.

Klain, G.S. $11964 \%$. Somantical power measured through the interference of words witth collornaming. American Journal of Psychology, 77,576-588.

Klorman. R., Weerts, T.C. Hastings, J.E., Melamed, B.G, \& Hang, P.J. (1974). Psychometric des scription of some specific fear questionnaires. Behavior Therapy, 5, 401-409.

La Heij. W. (1988). Components of Stroop-like interference in picture naming. Memory and Cognitior. $16,400-410$.

Landau, R.J. (1980). The role of semantic schemata in phobic word interpretation. Cognitive Therapy and Research, 4, 427-434.

Lang. Pal. $11977 \%$. Imagery in therapy: An information processing analysis of fear. Behavior Therapy, $8,862-886$

Lang. P.s. (1979). A biounformational theory of amotional imagery. Psychophysiology, 16, 495-512.

Lang, P.J. (1984). Cognition in emotion: Concept and action. In: C..E. Izard, J. Kagan. \& F. Zajonc Eds.h. Emotions, Cognition and Behavior. Cambridge University Press, New York. 
Lang, P.J. (1985). The cognitive psychophysiology of emotion: Fear and anxiety. In: A.H. Tuma, \& J.D. Maser (Eds.). Anxiety and the Anxiety Disorders. Eribaum Associates Publishers, Hillsdale, New Jersey.

Lavy, E.H. \& Hout; M.A. van den (1990). Thought suppression induces intrusions. Behavioural Psychotherapy, 18, 251-258.

Lavy, E. \& Hout, M. van den (1991). Does cognitive avoidance provoke attentional bias? Unpublished manuscript: Maasitricht: Limburg University, the Netherlands.

Lavy, E.H. \& Hout, M.A. van den (1993a). Attentional bias for appetitive cues: Effects of fasting in normal subjects. Behavioural and Cognitive Psychotherapy (in press).

Lavy, E.H. \& Hout, M.A. van den (1993b). Cognitive avoidance and attentional bials: Ciatsal relationships. Cognitive Therapy and Research (in press).

Lavy, E.H. \& Hout, M.A. van den (1993c). Selective attention evidenced by pictorial and linguisitic Stroop tasks. Behavior Therapy (in press).

Lavy, E.H. Hout, M. van den, \& Arntz, A. 11993d). Attentional bias and spider phobia: Conceptual and clinical issues. Behaviour Research and Therapy, 31, 17-24.

Lavy, E., Hout, M. van den, \& Arntz, A. (1993e). Attentional bias and facilitated escape: A pictorial test. Advances in Behaviour Research and Therapy, 15, 279-289.

Lavy, E., Oppen, P. van, \& Hout, M. van den (1993f). Selective processing of emotional information in obsessive compulsive disorder. Behaviour Research and Therapy (in press).

Lazarus, A.A. (1971). Behavior therapy and beyond. McGraw Hill, New York.

Lazarus, R.S. (1981). A cognitivist's reply to Zajonc on emotion and cognition. American Psychologist $, 36,222-223$.

Lazarus, R.S. 119821. Thoughts on the relations between emotion and cagnition. American Psychologist, $37,1019-1024$.

Lazarus, R.S. (1984). On the primacy of cognition. American Psychologist, 39, 124-129.

Lewis, A. (1942). Incidence of neurosis in England under war conditions . Lancet, 2, 175-183.

Litz, B.T. \& Keane, T.M. (1989), Information processing in anxiety disorders: Application to the understanding of post-traumatic stress disorder. Clinical Psychology Review, 9, 243-257.

Mackeod, C. (1990). Mood disorders and cognition. In: M.W. Eysenck (Ed.). Cognitive Psychology: An International Review. John Wiley and Sons, Chichester.

Macleod, C. (1991). Half a century of research on the Stroop effect: An integrative review. Psycho logical Bulletin, 109, 163-203.

MacLeod, C. \& Hagan, R. (1992). Individual differences in the selective processing of threatening information, and emotional responses to a stressfull life event. Behaviour Research and Therapy. $30,151-161$.

MacLeod, C. \& Mathews, A. (1988). Anxiety and the allocation of attention to threat. The Quarterly Jaurnal of Experimental Psychology, 40A, 653-670.

MacLeod, C. \& Mathews, A. 119911. Biased cognitive operations in anxiety: Accessibility of information ar assignment of processing priorities? Behaviour Research and Therapy, 29, 599*610.

Macleod, C. Mathews, A., \& Tata, P. (1986). Attentional bias in emotional disorders. Journat of Abnormal Psychology, 95, 15-20.

MacLeod, C. \& Rutherford, E.M. (1992). Anxiety and the seliective processing of emotional information: Mediating roles of awareness, trait and state variables, and personal relevance of stimulus materials. Behaviour Research and Therapy, 30, 479-491.

Marcel, A.J. (1983). Conscious and unconscious perception: Experiments on visual masking and word recognition. Cognitive Psychology, 15, $197-237$.

Marks. I.M. (1975). Behavioural treatments of phobic and obsessive-compulsive disorders: a critical appraisal. In: M. Hertsen, R.M. Eisler, \& P.M. Miller (Eds.). Progress in behavior modification (wol. 1). Academic Press, New York.

Marks I.M. (1987). Fears, phobias and rituals: panic, anxiety and their disorders, Oxford University Press, Oxford.

Marks, I. \& Mathews, A. (1979). Brief standard self-rating for phobic patients. Behaviour Research and Therapy $17,263-267$.

Martin, M., Horder, P., \& Jones, G.V. (1992). Integral bias in naming of phobia-related words. Cognition and Emotion, 6, 479-486.

Martin, M. Williams, R. \& Clark, D.M. (1991). Does anxiety lead to selective processing of threatrelated information? Behaviour Research and Therapy, 29, 147-160. 
Mathews, A. (1988). Anxisty and the processing of threatening information. In: V. Hamiltoin, G.H. Bower, \& N.H. Frijda (Eds.). Cognitive Perspectives on Emotion and Motivation. Kluwer Academic Publishers, Dordrecht.

Mathews, A. (1989). Cagnitive aspects of the etiology and phenomenology of anxiety disorders. In: P. Emmelkamp, W. Everaerd, F. Kraaimaat, \& M. van Son (Eds.). Fresh Perspectives on Asxiety Disorders. Swets North America, New York.

Mathews, A. \& Eysenck, M.W. 11987). Clinical anxiety and cognition. In: H.J. Eysenck, \& 1. Martin (Eds.). Theoretical foundations of behavior therapy. Plenum Press, New York.

Mathews, A. \& Klug, F. 119931. Emotionality and interference with color-naming in anxiety. Behaviour Research and Therapy, 31, 57-62.

Mathews, A. \& MacLeod, C. (1985). Selective processing of threat cues in anxiety states. Behaviour Research and Therapy, 23,563-569.

Mathews, A. \& MacLeod, C. (1986). Discrimination of threat cues without awareness in anxiety states. Jourmal of Abnormal Psychology, 95, 131-138.

Mathews, A. \& Macleod, C. 11987). An information-processing approach to anxiety. Journal of Cagnitive Psychotherapy: An international Quarteriy, 1, 105-115.

Mathews, A., May, J., Mogg, K., \& Eysenck, M. (1990). Attentional bias in anxiety: Selective search or defective filtering? Journal of Abnormal Psychology, 99, 166-173.

Mathews, A., Mogg, KK., May, J. \& Eysenck, M. (1989a). Implicit and explicit memory bias in anxiety. Journal of Abnormal Psychology, 98, 236-240.

Mathews, A., Richards, A., \& Eysenck, M. (1989b). Interpretation of homophones related to threat in anxiety states. Journal of Abnormal Psychology, 98, 31-34.

Mckenna, F.P. (1986). Effects of unattended emotional stimuli on color-naming performance. Current Psychological Research \& Reviews, 5, 3-9.

McNally, R.J. \& Foa, E.B. 11987 . Cognition and agoraphobia: Bias in the interpretation of threat. Cognitive Therapy and Research, 11, 567-581.

McNally; R.J., Foa, E.B., \& Donnell, C.D. (1989). Memory bias for anxiety information in patients with panic disorder. Cognition and Emotion, 3, 27-44,

McNally, R.J., Kaspi, S.P., Riemann, B.C., \& Zeitlin, S.B. (1990a). Selective processing of threat cues in post-traumatic stress disorder. Journal of Abnormal Psychology, 99, 398-402.

McNally, R.J., Riemann, B.C., \& Kim, E. (1990b). Selective processing of threat cues in panic disorder. Behaviour Research and Therapy, 28, 407-412.

McNally, R.J., Riemann, B.C., Louro, C.E., Lukach, B.M., \& Kim, E. (1992). Cognitive processing of emotional information in panic disorder. Behaviour Research and Therapy, 30, 143-149.

McNicol, D. (1972). A primer of signal detection theory. Allen and Unwin, London.

Meichenbaum, D. (1975). A self-instructional approach to stress management: A proposal for stress inoculation training. in: C.D. Spielberger, \& I.G. Sarason (Eds.). Stress and Anxiety (vol. 1). John Wiley and Sons, New York.

Meichanbaum, D. (1977). Cognitive bohavior madification: An integrative approach. General Learming Press, New York.

Merckelbach, H., Hout, W. van, Hout, M. van den, \& Lavy, I. (1990a). Aandachtsprocessen bij angst: Enkele kritische notities. Nederlands Tijdschrift voor de Psychologie, 45, 168-175.

Marckelbach, $H_{i}$, Hout, W. van, Jong, P. de, \& Hout, M. van den (1990b). Classical conditioning and attentional bias. Journal of Behaviour Research and Experimental Psychiatry, 21, 185-191.

Merckelbach, H., Kenemans, J.L., Dijkstra, A., \& Schouten, E. 11993). No attentional bias for picto ral stimuli in spider fearful subjects. Psychopathology and Behavioral Assessment in press).

Merckelbach, H. Muris, P. Hout, M. van den, \& Jong, $P$. de $\{1991\}$. Rebound effects of thought suppression: Instruction-dependent? Behavioural PSychotherapy, 19, 225-238.

Mineka, S., Davidson, M., Cook, M., \& Keir, R. (1984). Observational conditioning of snake fear in thesus monkeys. Journal of Abnormal Psychology, 93, 355-372.

Mogg, K. \& Marden, B. 11990). Processing of emotional information in anxious subjects. British Journal of Clinical Psychalogy, 29, 227-229.

Mogg, K. \& Mathews, A. (1990). Is there al self-referent mood-congruent recall bias in anxiety? Behawiour Research and Therapy, 28, 91-92.

Mogg, K., Mathews, A., \& Eysenck, M. (1992). Attentional bias to threat in clinical anxilety states. Cognition and Emotion, 6, 149-159. 
Mogg, K., Mathews, A., Eysenck, M., \& May, J. (1991a). Biased cognitive operations in anxilety: Artefact, processing priorities or attentional search? Behoviour Research and Therapy, 29, 459467.

Mogg, K., Mathews, A., May, J., Grove, M., Eysenck, M., \& Weinman, J. (1991b). Assessment of cognitive bias in anxiety and depression using a colour perception task. Cognition and Emotion, 5 , $221-238$.

Mogg, K., Mathews, A., \& Weinman, J. (1987). Memory bias in clinical anxiety. Journal of Abnormal Psychology, 96, 94-98.

Mogg, K., Mathews, A., \& Weinman, J. (1989). Selective processing of threat cues in anxiety states: A replication. Behaviour Research and Therapy, 27, 317-323.

Moray, N. (1959). Attention and dichotic listening: Effect of cues and the influence of instructions. Quarterly Journal of Experimental Psychology, 11, 56-60.

Morris, L.W., Davis, M.A., \& Hutching, C.H. (1981). Cognitive and emotional components of anxiety: Literature review and a revised worry-emotionality scale. Journal of Educational Psychology. $73,541-555$.

Mowrer, O.H. (1960). Learning theory and behavior. Wiley and Sons, New York.

Nielsen, S.L. \& Sarason, I.G. 119811. Emotion, personality and selective attention. Journal of Personality and Social Psychology, 41, 945-960.

Nisbett, R.E. \& Wilson, T. (1977). Telling more than we can know: Verbal reports on mental processes. Psychological Review, 84, 231-259.

Nunn, J.D., Stevenson, R.J., \& Whalan, G. (1984). Selective memory effects in agoraphobic patients. British Journal of Clinical Psychology, 23, 195-201.

Öhman, A. (1993). Fear and anxiety as emotional phenomena: Clinical phenomenology, evolutionary perspectives, and information processing mechanisms. In: M. Lewis \& J.M. Haviland (Eds.). Handbook of emotions. Guilford publications, New York.

Oppen, P. van \& Arntz, A. (1993). Cognitive therapy with obsessive compulsive disorders. Behaviour Research and Therapy (in press).

Orlemans, J.W.G. (1985). Inleiding tot de gedragstherapie. Van Loghum Slaterus B.V., Deventer.

Öst, L.G. (1989). One-session treatment for specific phobias. Behaviaur Research and Therapy, 27. $1-7$.

Overduin, J., Jansen, A., \& Louwerse, E. (1993). Stroop retardation and food intake. Submitted for publication.

Parkinson, L. \& Rachman, S. 11981). Part II. The nature of irtrusive thoughts. Advances in Behaviour Research and Therapy, 3, 101-110.

Pearce, J. \& Morley, S. (1989). An experimental investigation of the construct validity of the MeGill Pain Questionnaire. Pain, 39, 115-121.

Polivy, J., Herman, C.P., \& Warsch, S. (1978). Internal and external components of emotionality in restrained and unrestrained eaters. Journal of Abnormal Pswchology, 87, 197-204.

Power, M.J. (1991). Cognitive science and behavioural psychotherapy: Where behaviour was, there shall cognition be? Behavioural Psychotherapy, 19, 20-41.

Pratto, F. \& John, O.P. (1991). Automatic vigilance: The attention-grabbing power of negative social information. Journal of Personality and Social Psychology, 61, 380-391.

Rachman, S. (1971). Obsessional ruminations. Behaviour Research and Therapy, 9, 229-235.

Rachman, S. (1976). The passing of the two-stage theory of fear and avoidance: fresh possibilities. Behaviour Research and Therapy, 14, 125-131.

Rachman, S. (1977). The conditioning theory of fear-acquisition: a critical examination. Behaviour Research and Therapy, $15,375 * 387$.

Rachman, S. (1978). An anatomy of obsessions. Behaviour Analysis and Madification, 2, 253-278.

Rachman, S. (1981). Part I. Unwanted intrusive cognitions. Advances in Behaviour Research and Therapy, 3, 89-99.

Rachman, S. (1991). Neo-conditioning and the classical theory of fear acquisition. Clinical Psychology Review, 11, 155-173.

Rachman, S. \& De Silva, P. (1978). Abnormal and normal obsessions. Behawiour Research and

Therapy, 16, 233-248.
Ray, C. (1979). Examination stress and performance on a color-word interference test. Perceptual and Motor Skills, 49, 400-402. 
Reisis, S. 119801. Pavtovin conditoning and human fear: An expectancy model. Bethavior Therapy, 11.380996 .

Resconf, A.A. Wagner, A.A. 119721. A theory of pavlowan conditioning: variations in the effectiweness of remforcement and nonreinforcement. In: A.H. Black, \& W.F. Prokasy (Eds.). Classïal conditioning /I, Current research and theory. Appleton-Century-Crofts, New York.

Richards, A. French, C.C. 19901, Central versus peripheral presentation of stimuli in an emotional Sitroop task. Anxiery flesearch, $3,41-49$.

Richards, A. M. Milwood, B. (1989). Colouridentification of differentially valenced words in anxiety. Cognition and Emotion, 3, $171-176$.

Richards, A. \& Whitaker. T.M. 119901. Effects of anxiety and mood manipulation in autobiographic memory: British Journal of Clinical Psychology, 29, 145-153.

Roelofs, A. (1992). The spreading-activation theory of lemma retrieval in speaking. Cognition, 42 , $107-142$.

Rusted, d. Dighton, K. 119911. Selective processing of threat related material by spider phobics in a prose recall task, Cognition and Emotion, 5, 123-132.

Safran, J.D. \& Greenbelg, L.S. (1988). Feeling, thinking, and acting: A cognitive framework for psychotherapy intagration. Journal of Cognitive Psychotherapy: An International Ouarterly, 2, 109 131 .

Salkovskis, P.M. (1985), Obsessional compulsive problems: A coginituve behavioural analysis. Behaviour Research and Therapy, 23,571-583.

Salkovskis, P.M. Harrison. J. (1984), Abnomal and normal obsessions - a replication. Behaviour Research and Therapy, 22, 549-552.

Sartory, G. (1983). The orienting response and psychopathology: Anxiety and phobias. In: D. Siddle (Ed.). Orienting and Habituation: Perspectives in Human Research. Wiley and Sons, Chichester.

Schotte, D.E., MoNally, R.J., \& Turner, M.L. (1990). A dichotic listening analysis of body weight concern in bullimia nervosa. International Journal of Eating Disorders, 9, 109.113.

Schulte, D. Künzel, R., Pepping, G., \& Schulte-Bahrenberg, T. (1992). Tailor-made versus standardized therapy of phobic patients. Advances in Behaviour Research and Therapy, 114, 67-92.

Seligman; M.E.P. 119711. Phobias and preparedness. Behawior Therapy "2, 307-320.

Singer, J.A. \& Salovey, P. (1988). Mood and memory: evaluating the network theory of affect. Clinical Psychologv Review, 8, $211-251$.

Small. S.A. \& Robins, C.J. (1988). The influence of induced depressed mood on visual recognition thresholds: Predictive ambiguity of associative network models of mood and cognition. Cognitive Therapy and Research, 12, 295-303.

Smorenburg, J.M. J, Ent, C.K. van der, \& Bonke, B. (1986). Note on the validity of the dutch statetralt anxiety inventory with surgical patients. Psychological Reports, 59, 1333-1334.

Staats, A.W. (1968). Learning, Janguage and cagnition. Holt, Rinehart and Winstron, New York.

Staats, A.W. (1975). Social behaviorism. Irvin-Dorsey Press, London.

Stever, R. Mejcen, A.M., Schwonckmezger, P., B Buchner, A. (1989). A latent state-trait anxiety model and its application to determine consistency and specificity coefficients. Anxiety Reserarch, $1.281-299$.

Stroop. J.R. (1935). Studies of interfarence in serial verbal reactions. Journal of Experimental Psy. chology, 13, 643-662.

Stoler, L.S. MCNally, R.J. 11991). Cognitive bias in symptomatic and recovered agoraphobics Behawour Research and Therapy, 29,539-545.

Strien, T. van, Firiters, J.E.R., Bergers, G.P.A. \& Defares, P.B. (1986). The Dutch Eating Behaviour Quastionneire (DEBQ) for assessment of restraint, emotional and external eating behaviour.. lnternetional Jowrial of Eating Disorders, 2, 295-315.

Suinn, R.M. (1976). Anxiety mainagement training to control general anxiety. In: J.D. Krumbolt $Z_{s} \&$ C.E. Thorensen (Eds.). Coumseling methods. Holt, Finethart \& Winston, New York.

Suinn, R.M. \& Alchardson, F. 1971). Anxiety management training: A nonspecific therapy program for anxiety control. Behavior Therapy, 2, 498-511.

Tesser, A. (1976). Thought and reality constrainst as determinants of attitude polarization. Journal of Research in Personality. 10, 183-194.

Trandel. D.V. \& McNally, R.J. (1987). Perception of threat cues in post-traumatic stress disorder: Semantic processing without awareness? Behaviour Research and Therapy, 25, 469 476. 
Uit den Boogaart, P.C. (1975). Woordfrequenties - In Geschreven en Gesproken Nedertands. Oosthoek. Scheltema and Holkema, Uitrecht.

Von Streblow, $H_{\text {. }}$, Hoffmann, J., \& Kasielke, E, (1985). Experimentalpsychologische Analyse von Gedächtnizprozessen bel Phobikern. Zeitschrift fö Psychology, 193, 147-161.

Wachtel, P.L. (1967). Conceptions of broad and narrow attention. Psychological Bulletin, 68, 417. 429.

Wachtel, P.L. (1968). Anxiety, attention and coping with threat. Journal of Abnormal Psychology, $73,137-143$.

Watson, J.B. \& Rayner, R. (1920). Conditioned emotional reactions. Journal of Experimental Psychology, 3, 1-14.

Watts, F.N. (1990). Aversion to personal body hair: A case in the integration of behavioural and interpretative methods. British Journal of Medical Psychology, 63, 335-340.

Watts, F.N. \& Coyle, K. (1992). Recall bias for stimulus and response anxiety words in spider phobics. Anxiety Research, 4, 315-324.

Watts, F.N. \& Dalgleish, T. 11991$)$. Memory for phobia-related words in spider phobics. Cognition and Emotion, 313-329.

Watts, F.N., McKenne, F.P., Sharrack, R., \& Trezise, L. (1986a). Colour naming of phobia-related words. British Journal of Psychology, 77, 97-108.

Watts F., Sharrock, R., \& Trezise, L. (1986b). Detail and elaboration in phobic inagery. Behavioural Psychotherapy, 14, 115-123.

Watts, F.N., Trezise, L., \& Sharrack, R. $(1986 \mathrm{c})$. Processing of phabic stimuli. British Journal of Clinical Psychalogy. 25, 253-261.

Wegner, D.M. (1989). White Bears and Other Unwanted Thoughts: Suppression, Obsession, and the Psychology of Mental Control. Viking Press, New York.

Wegner, D.M. \& Erber, R. (1992). The hyperaccessibility of suppressed thoughts. Journal of Personality and Social Psychology, 63, 903-912.

Wegner, D.M., Schneider, D.J., Carter, S.R., \& White, T.L. (1987). Paradoxical effects of thought suppression. Journal of Personality and Social Psychology ${ }^{53}, 5-13$.

Wegner, D.M., Short, J.W., Blake, A.W., \& Page, M.S. (1990). The suppression of exciting thoughts. Joumal of Personality and Social Psychology, 58, 409-418.

Williams, J.M.G. \& Broadbent, K. (1986). Distraction by emotional stimuli: Use of a Stroop task with suicide attempters. British Journal of Clinical Psychology, 25, 101-110.

Williams, J.M.G., Watts, F.N., MacLeod, C., \& Mathews, A. (1988). Cognitive psychology and emotional disorders. Wiley \& Sons, Chichester.

Wolpe, J. (1958). Psychotherapy by recipracal inhibition. Stanford University Press, California.

Yuksel, S., Marks, I.M., Rarmm, E., \& Gosh, A. (1984). Slow versus rapid exposure in vivo of phobics. Behavioural Psychotherapy, 12, 249-256.

Zajonc, R.B. (1980). Feelling and thinking - preferences need no inferences. American Psychologist, $35,151-175$

Zajonc, R.B. (1984). On the primacy of affect. American Psychalogist, 39, 117-123

Zeitlin, S.B. \& MCNally, R.J. 11991). Implicit and explicit memory bias for threat in post-traumatic stress disorder. Behaviour Research and Therapy, 29,451-457. 


\section{Dankwoord}

Aan het slot van dit proefschrift gekomen rest mij de aangename taak diegenen te bedanken, die een bijdrage hebben geleverd aan de totstandkoming van dit boekje.

Daarmee bedoel ik op de eerste plaats Marcel van den Hout, mijn promotor. Ik dank je voor alle wetenschappelijke vrijheid die ik als student en als AIO genoten heb. Je enthousiasme voor de meest uiteenlopende experimenten, je ruige onderzoeksplannen en je deskundig kommentaar op de vele kladversies van mijn schrijfsels maakten van mijn AlO jaren een buitengewoon leerzame periode. Ook al ben ik nu uit het wetenschapsbedrijf gestapt, de kennis en kunde die ik de afgelopen jaren van je meegekregen heb, waardeer ik zeer.

De kollega's van het onderzoeksprojekt Experimentele Psychopathologie will ik bedanken voor het voortreffelijke onderzoeksklimaat. Goede herinneringen bewaar ik aan de drie spinnenprojekten; een samenwerkingsverband met Arnoud Arntz, Peter de Jong, Harald Merckelbach, Dorien Walfs, Germie van den Berg, Anja Meijboom, Birgit Mayer, Arie Dijkstra, en een aantal tijdelijke student-assistenten. Ik will jullie graag bedanken voor de wetenschappelijke diskussies, de morele ondersteuning en de nodige gezelligheid.

Patricia van Oppen wil ik bedanken voor de jarenlange samenwerking aan het onderzoek naar de selektiviteit van aandachtsverstoringen bij dwangpatiënten. Ik heb er een grote bewondering aan overgehouden voor het geduld dat toegepast onderzoekers moeten opbrengen. Heidi Mertens ben ik erkentelijk voor de hulp, geboden bij het gedachtenonderdrukkingsexperiment.

Zonder technische ondersteuning was van dit onderzoek weinig terecht gekomen. Dank aan Theo van Aarts die het prachtige Stroop programma ontwikkelde, dat inmiddels nationale faam heeft verworven. Dank ook aan Ger van Wunnik die samen met mij de audiobanden heeft geskoord van het onderzoek bij dwangpatiënten.

Het is al vaker gezegd: zonder proefpersonen was dit proefschrift niet tot stand gekomen. Een grote groep mensen bleek steeds bereid zonder verdere toelichting de meest wonderlijke opdrachten uit te voeren. Al die anonieme proefpersonen wil ik bij deze hartelijk danken voor hun medewerking.

De $\mathrm{AlO}^{\prime}$ s en bestuursleden van het AlO netwerk Experimentele. Psychopathologie dank ik voor de gezellige en leerzame bijeenkomsten in hotel "Het Witte Huis". De kursussen van het netwerk waren voor mij de slagroom op het AlO-schap.

Mijn paranimfen Helga Nauta en Peter de Jong dank ik voor hun hulp bij de arganisatie van de $23 e$ december.

Tot slot wil ik het thuisfront bedanken. Allereerst mijn ouders, omdat zij mij van jongs af aan hebben gestimuleerd mijn hersens te gebruiken. Ik dank jullie voor alle mogelijkheden die jullie mij geboden hebben. Veel dank komt toe aan René. Niet voor niets heb ik dit boekwerkje aan jou opgedragen. Het uitvoeren van onderzoek is een frustrerende bezigheid; je vertrouwen in mijn kapaciteiten was de afgelopen jaren een grote steun tijdens mijn vele pessimistische buien. Dank voor al je geduld. 


\section{Curriculum vitae}

Edith Hanna (ldith) Lavy werd in Haarlem geboren op 26 oktober 1966. In 1985 behaalde zij het VWO diploma aan het Mendelcollege te Haarlem. In datzelfde jaar begon zij met de studie Gezondheidswetenschappen, afstudeerrichting Geestelijke Gezondheidkunde, aan de Rijksuniversiteit Limburg. Deze studie rondde zij in 1989 af.

Juli 1989 trad zij in dienst bij de vakgroep Geestelijke Gezondheidkunde van de Rijksuniversiteit Limburg. Het onderzoek waarop dit proefschrift is gebaseerd vond plaats tijdens dit vierjarig dienstverband als assistent in opleiding. Tijdens dit dienstverband volgde zij tevens de opleiding tot gedragstherapeut.

Sinds 1 juli 1993 heeft zij een tijdelijke aanstelling bij het PC Wijnkoperstraat, deeltijdbehandeling in Gorincherm van het APZ "De Grote Rivieren". 